\title{
Fine-tuning Monophosphine Ligands for Enhanced Enantioselectivity. Influence of Chiral Hemilabile Pendant Groups
}

\author{
Aibin Zhang and T. V. RajanBabu \\ Department of Chemistry, 100 W. $18^{\text {th }}$ Avenue, The Ohio State University, Columbus, OH \\ 43210
}

\section{Supporting Information}

General methods. Reactions requiring air-sensitive manipulations were conducted under an inert atmosphere of nitrogen by using Schlenk techniques or a Vacuum Atmospheres drybox. Methylene chloride and DMSO were distilled from calcium hydride under nitrogen and stored over molecular sieves. Tetrahydrofuran, hexane and benzene were distilled under nitrogen from sodium/benzophenone ketyl. 2-Bromobenzaldehyde, $(2 R, 3 R)$-butanediol, $(2 S, 3 S)$-butanediol, $(2 R, 5 R)$-hexanediol, $(2 S, 5 S)$-hexanediol, $(2 S, 4 S)$-pentanediol, $N, N$-diisopropylethylamine, 4 methylstyrene, 4-bromostyrene, 4-methoxystyrene and 2-methyl-2-butene were purchased from Acros. $\mathrm{LiAlH}_{4}(1 \mathrm{M}$ in diethyl ether), diethyl phosphite, styrene and 2-vinylnaphthalene were purchased from Aldrich. Allylic nickel bromide ${ }^{1}, \mathrm{Na}^{+}\left[\left[3,5-\left(\mathrm{CF}_{3}\right)_{2} \mathrm{C}_{6} \mathrm{H}_{3}\right]_{4} \mathrm{~B}\right]^{-}\left(\mathrm{Na}^{+} \mathrm{BARF}\right)^{2}, 4-$ isobutylstyrene ${ }^{3}, 6$-methoxy-2-vinylnaphthalene ${ }^{3}$ were prepared according to the literature. Ethylene (99.5\%) was purchased from Matheson Inc., and passed through Drierite before use. For ozonolysis, ozone gas was delivered using a Welsbach ozone generator. Analytical TLC was performed on E. Merck precoated $(0.25 \mathrm{~mm})$ silica gel $60 \mathrm{~F}_{254}$ plates. Flash column chromatography was carried out on silica gel 40 (Scientific Adsorbents Incorporated, Microns Flash). Gas chromatographic analyses were performed on a Hewlett-Packard 5890 equipped with an HP-ultra-1 crosslinked methyl silicone capillary column ( $25 \mathrm{~m}$ length $\mathrm{x} 0.2 \mathrm{~mm}$ i.d.) and a FID detector connected to a HP 3396 integrator. Helium was used as the carrier gas. Chiral gas chromatographic separations were accomplished using Chirasil-L-Val on WCOT fused silica (25 $\mathrm{m} \times 0.25 \mathrm{~mm}, 0.12 \mathrm{~mm}$ film thickness) capillary GC column purchased from Chrompack. Enatiomeric excesses of all compounds were determined by HPLC using a Daicel Chiralcel OJ column using hexane/isopropanol as solvents where base-line separation was obtained. Optical rotations were recorded in solution on a Perkin-Elmer Model 241 polarimeter at the sodium 
$\mathrm{D}$ line in chloroform. The optical rotations of ligands were not measured because of their air-sensitivity.

\section{Preparation of Ligands}

Preparation of $(2 R, 5 R)$-hexanediol cyclic sulfate ${ }^{4}$ :

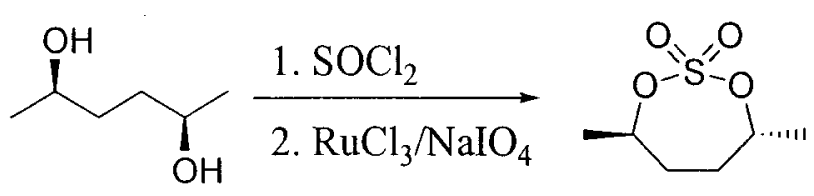

To a solution of $(2 R, 5 R)$-hexanediol $(1.18 \mathrm{~g}, 10 \mathrm{mmol})$ in $\mathrm{CCl}_{4}(7 \mathrm{~mL})$ was added via syringe thionyl chloride $(1.2 \mathrm{~mL}, 16.5 \mathrm{mmol})$. The resulting brown solution was then heated at reflux for $1.5 \mathrm{~h}$. After the solution was cooled to r.t., the reaction mixture was concentrated on a rotary evaporator to afford a brown oil. The oil was then dissolved in a mixture of $\mathrm{CCl}_{4}(7 \mathrm{~mL}), \mathrm{CH}_{3} \mathrm{CN}(7 \mathrm{~mL})$ and $\mathrm{H}_{2} \mathrm{O}(10 \mathrm{~mL})$ and the mixture was cooled to $0^{\circ} \mathrm{C}$. To the cold mixture was added $\mathrm{RuCl}_{3} 3 \mathrm{H}_{2} \mathrm{O}(7.3 \mathrm{mg}, 0.028 \mathrm{mmol})$, followed by $\mathrm{NaIO}_{4}(4.43 \mathrm{~g}, 20.7 \mathrm{mmol})$. The reaction was allowed to stir at r.t. for $1 \mathrm{~h} . \mathrm{H}_{2} \mathrm{O}(40 \mathrm{~mL})$ was added, the reaction mixture was extracted with diethyl ether $(4 \times 40 \mathrm{~mL})$ and the combined extracts were washed with brine. After drying over $\mathrm{MgSO}_{4}$ and the solution was filtered through a pad of silica gel, the colorless solution was concentrated to yield white solid $(1.57 \mathrm{~g}, 87 \%)$ which was used without further purification. ${ }^{1} \mathrm{H} \mathrm{NMR}\left(\mathrm{CDCl}_{3}\right.$, $250 \mathrm{MHz}) \delta 4.95-4.75(\mathrm{~m}, 2 \mathrm{H}), 2.10-1.80(\mathrm{~m}, 4 \mathrm{H}), 1.46(\mathrm{~d}, J=6.35 \mathrm{~Hz}, 6 \mathrm{H})$.

\section{Preparation of $(2 S, 5 S)$-hexanediol cyclic sulfate ${ }^{4}$ :}

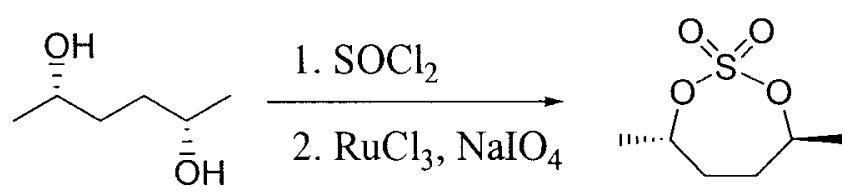

The procedure is the same as the above. Starting from $(2 S, 5 S)$-hexanediol $(1.18 \mathrm{~g}, 10$ mmol) to get $1.55 \mathrm{~g}(86 \%)$ of $(2 \mathrm{~S}, 5 \mathrm{~S})$-hexanediol cyclic sulfate. ${ }^{1} \mathrm{H} \mathrm{NMR}\left(\mathrm{CDCl}_{3}, 250\right.$ MHz) $\delta 4.90-4.65(\mathrm{~m}, 2 \mathrm{H}), 2.10-1.80(\mathrm{~m}, 4 \mathrm{H}), 1.41$ (d, $J=6.35 \mathrm{~Hz}, 6 \mathrm{H})$.

Preparation of 2-(2-bromophenyl)-(4R,5R)-dimethyl-[1,3]dioxolane ${ }^{5}$ : 


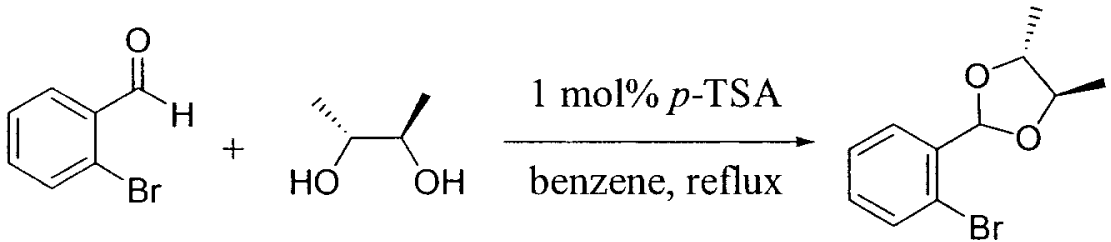

To a solution of 2-bromobenzaldehyde $(1.76 \mathrm{~g}, 9.5 \mathrm{mmol})$ and $(2 R, 3 R)$-butanediol $(0.94$ $\mathrm{g}, 10.4 \mathrm{mmol})$ in benzene $(5 \mathrm{~mL})$ was added $p$-toluenesulfonic acid monohydrate $(19 \mathrm{mg}$, $0.1 \mathrm{mmol}$ ). The resulting solution was heated under reflux with Dean-Stark trap for $5 \mathrm{~h}$. The reaction mixture was poured into aqueous $\mathrm{NaHCO}_{3}$ and extracted with diethyl ether. The organic layers were combined, dried and concentrated to afford the crude product which was purified by flash chromatography (eluent: hexane:ethyl acetate $=40: 1$ ) to give $2.04 \mathrm{~g}(84 \%)$ of 2-(2-bromophenyl)-(4R,5R)-dimethyl-[1,3]dioxolane. ${ }^{1} \mathrm{H} \mathrm{NMR}\left(\mathrm{CDCl}_{3}\right.$, $250 \mathrm{MHz}) \delta 7.62(\mathrm{dd}, J=7.75,1.75 \mathrm{~Hz}, 1 \mathrm{H}), 7.53(\mathrm{dd}, J=7.75,1.03 \mathrm{~Hz}, 1 \mathrm{H}), 7.32(\mathrm{td}, J$ $=7.50,0.83 \mathrm{~Hz}, 1 \mathrm{H}), 7.18(\mathrm{td}, J=7.75,1.78 \mathrm{~Hz}, 1 \mathrm{H}), 6.22(\mathrm{~s}, 1 \mathrm{H}), 3.90-3.70(\mathrm{~m}, 2 \mathrm{H})$, $1.38(\mathrm{t}, J=5.50 \mathrm{~Hz}, 3 \mathrm{H}), 1.32(\mathrm{t}, J=5.50 \mathrm{~Hz}, 3 \mathrm{H}) ;{ }^{13} \mathrm{C} \mathrm{NMR}\left(\mathrm{CDCl}_{3}, 62.9 \mathrm{MHz}\right) \delta$ $16.75,17.06,78.66,80.43,101.47,122.93,127.43,128.09,130.46,132.91,137.31$.

Preparation of [2-((4R,5R)-dimethyl-[1,3]dioxolan-2-yl)-phenyl]-phosphonic acid diethyl ester ${ }^{6}$ :

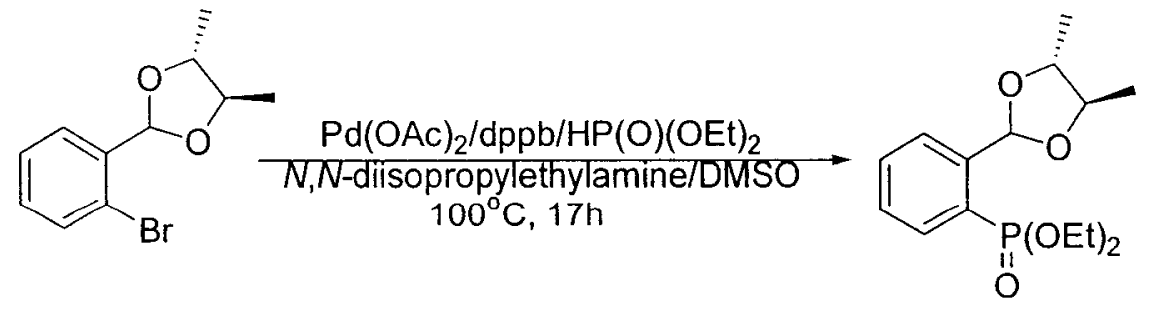

A mixture of 2-(2-bromophenyl)-(4R,5R)-dimethyl-[1,3]dioxolane $(1.99 \mathrm{~g}, 7.74 \mathrm{mmol})$, diethylphosphite (2.3 mL, $17.8 \mathrm{mmol}), \mathrm{Pd}(\mathrm{OAc})_{2}(87 \mathrm{mg}, 0.39 \mathrm{mmol})$, dppb (165 mg, $0.39 \mathrm{mmol})$ and $N, N$-diisopropylethylamine $(5.4 \mathrm{~mL}, 31 \mathrm{mmol})$ in DMSO $(11 \mathrm{~mL})$ was heated with stirring at $100^{\circ} \mathrm{C}$ under nitrogen atmosphere overnight. After the mixture was cooled to r.t., $10 \mathrm{~mL}$ of water was added slowly and the mixture was extracted with ethyl acetate. The extracts were combined, washed with water, dried over $\mathrm{MgSO}_{4}$ and concentrated in vacuo to give a crude oil which was purified by column chromatography 
(eluent: hexane:ethyl acetate $=4: 1)$ to afford $1.28 \mathrm{~g}(53 \%)$ of [2-((4R,5R)-dimethyl[1,3]dioxolan-2-yl)-phenyl]-phosphonic acid diethyl ester and $100 \mathrm{mg}(5 \%)$ of 2-(2bromophenyl)-(4R,5R)-dimethyl-[1,3]dioxolane was recovered. ${ }^{1} \mathrm{H}$ NMR $\left(\mathrm{CDCl}_{3}, 250\right.$ MHz) $\delta 7.83(\mathrm{dd}, J=14.0,7.75 \mathrm{~Hz}, 1 \mathrm{H}), 7.70(\mathrm{t}, J=5.50 \mathrm{~Hz}, 1 \mathrm{H}), 7.46(\mathrm{t}, J=7.50 \mathrm{~Hz}$, $1 \mathrm{H}), 7.40-7.25(\mathrm{~m}, 1 \mathrm{H}), 6.43(\mathrm{~s}, 1 \mathrm{H}), 4.15-3.85(\mathrm{~m}, 4 \mathrm{H}), 3.80-3.60(\mathrm{~m}, 2 \mathrm{H}), 1.40-1.05(\mathrm{~m}$, $12 \mathrm{H}) ;{ }^{13} \mathrm{C} \mathrm{NMR}\left(\mathrm{CDCl}_{3}, 62.9 \mathrm{MHz}\right) \delta 15.90\left(\mathrm{~d}, J_{\mathrm{P}, \mathrm{C}}=6.60 \mathrm{~Hz}\right), 16.62,17.06,61.86(\mathrm{~d}$, $\left.J_{\mathrm{P}, \mathrm{C}}=5.2 \mathrm{~Hz}\right), 78.62,80.13,98.96\left(\mathrm{~d}, J_{\mathrm{P}, \mathrm{C}}=3.8 \mathrm{~Hz}\right), 125.18,127.30\left(\mathrm{~d}, J_{\mathrm{P}, \mathrm{C}}=13.5 \mathrm{~Hz}\right)$, $128.30\left(\mathrm{t}, J_{\mathrm{P}, \mathrm{C}}=14.5 \mathrm{~Hz}\right), 132.42\left(\mathrm{~d}, J_{\mathrm{P}, \mathrm{C}}=2.8 \mathrm{~Hz}\right), 133.49\left(\mathrm{~d}, J_{\mathrm{P}, \mathrm{C}}=9.1 \mathrm{~Hz}\right), 141.65(\mathrm{~d}$, $\left.J_{\mathrm{P}, \mathrm{C}}=10.1 \mathrm{~Hz}\right) ;{ }^{31} \mathrm{P} \mathrm{NMR}\left(\mathrm{C}_{6} \mathrm{D}_{6}, 101.3 \mathrm{MHz}\right) \delta 18.94$.

Preparation of 2-[(4R,5R)-dimethyl-[1,3]dioxolan-2-yl]-phenyl-phosphane ${ }^{7}$ :
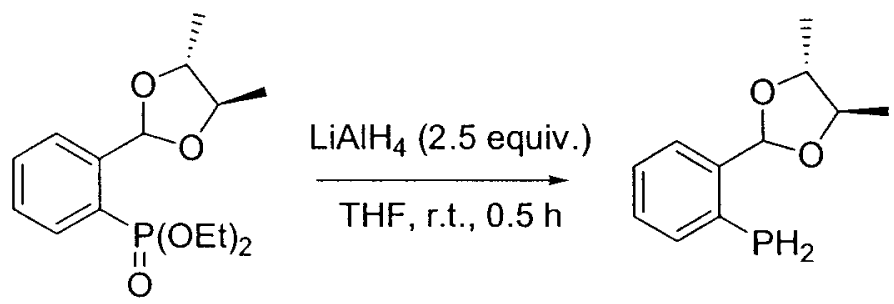

To a solution of [2-((4R,5R)-dimethyl-[1,3]dioxolan-2-yl)-phenyl]-phosphonic acid diethyl ester $(314 \mathrm{mg}, 1 \mathrm{mmol})$ in THF $(10 \mathrm{~mL})$ was added a solution of $\mathrm{LiAlH}_{4}(2.5 \mathrm{~mL}$, $1 \mathrm{M}$ in ether, $2.5 \mathrm{mmol}$ ) slowly at r.t. in drybox. After the addition, the reaction mixture was stirred for additional $30 \mathrm{~min}$ at r.t. The reaction was quenched with minimum amount of saturated aqueous $\mathrm{NH}_{4} \mathrm{Cl}$ and the solvent was removed under reduced pressure. Dry benzene $(2 \times 5 \mathrm{~mL})$ was added to remove water azetropically from the solid residue. The solid was extracted with hexane three times and the extracts were combined and concentrated to afford a crude oil which was purified by flash column chromatography (eluent: hexane:ether $=40: 1)$ to get $52 \mathrm{mg}(25 \%)$ of 2-[(4R,5R)-dimethyl-[1,3]dioxolan2-yl]-phenyl-phosphane. ${ }^{1} \mathrm{H}$ NMR $\left(\mathrm{CDCl}_{3}, 250 \mathrm{MHz}\right) \delta$ 7.55-7.35 (m, 2H), 7.25-7.05(m, $2 \mathrm{H}), 6.03\left(\mathrm{~d}, J_{\mathrm{P}, \mathrm{H}}=2.0 \mathrm{~Hz}, 1 \mathrm{H}\right), 3.90\left(\mathrm{~d}, J_{\mathrm{P}, \mathrm{H}}=206 \mathrm{~Hz}, 2 \mathrm{H}\right), 3.85-3.65(\mathrm{~m}, 2 \mathrm{H}), 1.29(\mathrm{~d}$, $J=4.50 \mathrm{~Hz}, 3 \mathrm{H}), 1.23(\mathrm{~d}, J=4.50 \mathrm{~Hz}, 3 \mathrm{H}) ;{ }^{13} \mathrm{C} \mathrm{NMR}\left(\mathrm{CDCl}_{3}, 62.9 \mathrm{MHz}\right) \delta 16.79$, $17.35,78.60,80.62,101.80\left(\mathrm{~d}, J_{\mathrm{P}, \mathrm{C}}=9.0 \mathrm{~Hz}\right), 127.00\left(\mathrm{~d}, J_{\mathrm{P}, \mathrm{C}}=3.4 \mathrm{~Hz}\right), 128.28,128.73(\mathrm{~d}$, $\left.J_{\mathrm{P}, \mathrm{C}}=3.2 \mathrm{~Hz}\right), 128.92,136.93\left(\mathrm{~d}, J_{\mathrm{P}, \mathrm{C}}=6.4 \mathrm{~Hz}\right), 140.92\left(\mathrm{~d}, J_{\mathrm{P}, \mathrm{C}}=10.9 \mathrm{~Hz}\right) ;{ }^{31} \mathrm{P} \mathrm{NMR}$ $\left(\mathrm{C}_{6} \mathrm{D}_{6}, 101.3 \mathrm{MHz}\right) \delta-126.08$. 
Preparation of 2-[2-((2S,5S)-dimethyl-phospholan-1-yl)-phenyl]-(4R,5R)-dimethyl$[1,3]$ dioxolane $(1 \mathrm{a})^{8}$ :

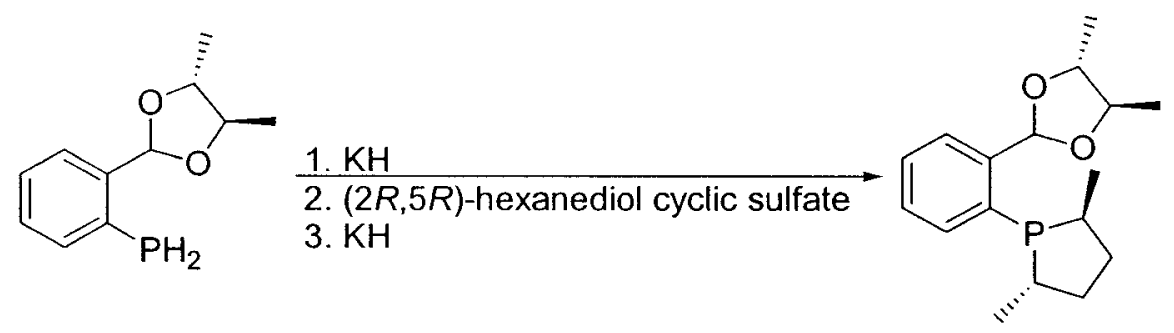

To a solution of 2-[(4R,5R)-dimethyl-[1,3]dioxolan-2-yl]-phenyl-phosphane (52 mg, 0.25 mmol $)$ in THF $(10 \mathrm{~mL})$ was added $\mathrm{KH}\left(10.3 \mathrm{mg}, 0.26 \mathrm{mmol}\right.$ in $0.5 \mathrm{~mL}$ of THF) at $-30^{\circ} \mathrm{C}$ in drybox. The mixture was allowed to warm to r.t. and stirred for $1.5 \mathrm{~h}$. To the resulting solution was added slowly a solution of $(2 R, 5 R)$-hexanediol cyclic sulfate ( $45 \mathrm{mg}$, $0.25 \mathrm{mmol})$ in THF $(1 \mathrm{~mL})$ and the mixture was stirred for $2 \mathrm{~h}$ at r.t. Finally $\mathrm{KH}(10.3 \mathrm{mg}$, $0.26 \mathrm{mmol}$ in $0.5 \mathrm{~mL}$ of THF) was added at r.t. and stirred for additional $2 \mathrm{~h}$. The reaction was quenched with a few drops of methanol and the solvents were removed under vacuum to get a crude oil. Purification by column chromatography (eluent:hexane:ether $=40: 1)$ afforded $31 \mathrm{mg}(42 \%)$ of 2-[2-((2S,5S)-dimethylphospholan-1-yl)-phenyl]-(4R,5R)-dimethyl-[1,3]dioxolane. ${ }^{1} \mathrm{H}$ NMR $\left(\mathrm{C}_{6} \mathrm{D}_{6}, 250 \mathrm{MHz}\right) \delta$ 7.98 (ddd, $J=7.75,3.25,1.48 \mathrm{~Hz}, 1 \mathrm{H}$ ), 7.35 (dt, $J=7.50,1.65 \mathrm{~Hz}, 1 \mathrm{H}$ ), 7.24 (td, $J=$ 7.50, $1.30 \mathrm{~Hz}, 1 \mathrm{H}), 7.18-7.08(\mathrm{~m}, 2 \mathrm{H}), 3.85-3.50(\mathrm{~m}, 2 \mathrm{H}), 2.60-2.42(\mathrm{~m}, 1 \mathrm{H}), 2.40-2.18$ $(\mathrm{m}, 1 \mathrm{H}), 2.14-1.92(\mathrm{~m}, 1 \mathrm{H}), 1.92-1.73(\mathrm{~m}, 1 \mathrm{H}), 1.55-1.35(\mathrm{~m}, 1 \mathrm{H}), 1.30-1.10(\mathrm{~m}, 4 \mathrm{H})$, $1.08(\mathrm{~d}, J=5.80 \mathrm{~Hz}, 3 \mathrm{H}), 1.04(\mathrm{~d}, J=6.0 \mathrm{~Hz}, 3 \mathrm{H}), 0.91(\mathrm{dd}, J=9.75,7.0 \mathrm{~Hz}, 3 \mathrm{H}) ;{ }^{13} \mathrm{C}$ $\operatorname{NMR}\left(\mathrm{C}_{6} \mathrm{D}_{6}, 62.9 \mathrm{MHz}\right) \delta 16.53\left(\mathrm{~d}, J_{\mathrm{P}, \mathrm{C}}=1.3 \mathrm{~Hz}\right), 16.94,17.52,21.03(\mathrm{~d}, J=36.5 \mathrm{~Hz})$, $35.17\left(\mathrm{~d}, J_{\mathrm{P}, \mathrm{C}}=7.6 \mathrm{~Hz}\right), 35.36\left(\mathrm{~d}, J_{\mathrm{P}, \mathrm{C}}=5.0 \mathrm{~Hz}\right), 36.70\left(\mathrm{~d}, J_{\mathrm{P}, \mathrm{C}}=2.1 \mathrm{~Hz}\right), 37.17\left(\mathrm{~d}, J_{\mathrm{P}, \mathrm{C}}=\right.$ $2.6 \mathrm{~Hz}), 79.10,80.52,100.66\left(\mathrm{~d}, J_{\mathrm{P}, \mathrm{C}}=31.5 \mathrm{~Hz}\right), 127.06\left(\mathrm{~d}, J_{\mathrm{P}, \mathrm{C}}=5.5 \mathrm{~Hz}\right), 128.19$, $128.72,132.74\left(\mathrm{~d}, J_{\mathrm{P}, \mathrm{C}}=3.7 \mathrm{~Hz}\right), 136.75\left(\mathrm{~d}, J_{\mathrm{P}, \mathrm{C}}=36.2 \mathrm{~Hz}\right), 145.22\left(\mathrm{~d}, J_{\mathrm{P}, \mathrm{C}}=20.7 \mathrm{~Hz}\right)$;

${ }^{31} \mathrm{P}$ NMR $\left(\mathrm{C}_{6} \mathrm{D}_{6}, 101.3 \mathrm{MHz}\right) \delta$-5.35; HRMS (ESI) $\mathrm{m} / \mathrm{z} 293.1649\left([\mathrm{M}+\mathrm{H}]^{+}\right.$, exact mass calcd for $\mathrm{C}_{17} \mathrm{H}_{26} \mathrm{O}_{2} \mathrm{P} 293.1665$ ).

Preparation of 2-[2-((2S,5S)-diethyl-phospholan-1-yl)-phenyl]-(4R,5R)-dimethyl$[1,3]$ dioxolane $(1 \mathrm{~b})^{8}$ : 


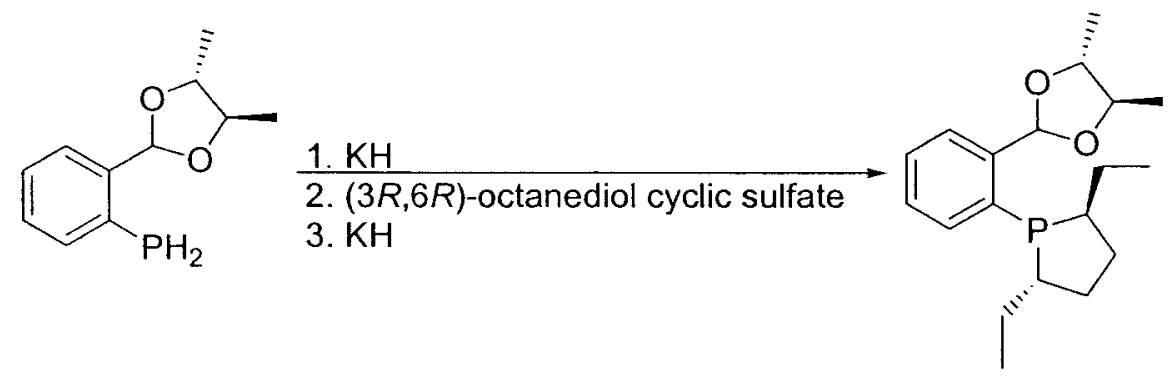

The procedure is the same as the above. Starting from 2-[(4R,5R)-dimethyl[1,3]dioxolan-2-yl]-phenyl-phosphane $(58 \mathrm{mg}, 0.28 \mathrm{mmol})$ and $(3 R, 6 R)$-octanediol cyclic sulfate $(57.4 \mathrm{mg}, 0.28 \mathrm{mmol})$ to get $40 \mathrm{mg}(45 \%)$ of 2-[2-((2S,5S)-diethyl-phospholan-1yl)-phenyl]-(4R,5R)-dimethyl-[1,3]dioxolane. ${ }^{1} \mathrm{H}$ NMR $\left(\mathrm{C}_{6} \mathrm{D}_{6}, 250 \mathrm{MHz}\right) \delta 8.0-7.9(\mathrm{~m}$, $1 \mathrm{H}), 7.39(\mathrm{dt}, J=6.0,1.5 \mathrm{~Hz}, 1 \mathrm{H}), 7.30-7.05(\mathrm{~m}, 3 \mathrm{H}), 3.75-3.45(\mathrm{~m}, 2 \mathrm{H}), 2.40-2.25(\mathrm{~m}$, 1H), 2.15-1.80 (m, 3H), 1.75-1.35 (m, ca. $5 \mathrm{H}), 1.25-1.10(\mathrm{~m}, \mathrm{ca} .1 \mathrm{H}), 1.07$ (d, $J=6.0 \mathrm{~Hz}$, $3 \mathrm{H}), 1.03(\mathrm{~d}, J=6.0 \mathrm{~Hz}, 3 \mathrm{H}), 0.91(\mathrm{t}, J=7.25 \mathrm{~Hz}, 3 \mathrm{H}), 0.80(\mathrm{t}, J=7.13 \mathrm{~Hz}, 3 \mathrm{H}) ;{ }^{13} \mathrm{C}$ $\operatorname{NMR}\left(\mathrm{C}_{6} \mathrm{D}_{6}, 62.9 \mathrm{MHz}\right) \delta 14.35\left(\mathrm{~d}, J_{\mathrm{P}, \mathrm{C}}=1.7 \mathrm{~Hz}\right), 14.50\left(\mathrm{~d}, J_{\mathrm{P}, \mathrm{C}}=5.4 \mathrm{~Hz}\right), 16.93,17.51$, $24.50,29.33\left(\mathrm{~d}, J_{\mathrm{P}, \mathrm{C}}=33.1 \mathrm{~Hz}\right), 34.35\left(\mathrm{~d}, J_{\mathrm{P}, \mathrm{C}}=1.5 \mathrm{~Hz}\right), 34.52\left(\mathrm{~d}, J_{\mathrm{P}, \mathrm{C}}=3.2 \mathrm{~Hz}\right), 43.06$ $\left(\mathrm{d}, J_{\mathrm{P}, \mathrm{C}}=12.6 \mathrm{~Hz}\right), 45.25\left(\mathrm{~d}, J_{\mathrm{P}, \mathrm{C}}=11.3 \mathrm{~Hz}\right), 79.06,80.52,100.65\left(\mathrm{~d}, J_{\mathrm{P}, \mathrm{C}}=33.5 \mathrm{~Hz}\right)$, $127.19\left(\mathrm{~d}, J_{\mathrm{P}, \mathrm{C}}=5.8 \mathrm{~Hz}\right), 128.10,128.84,133.86\left(\mathrm{~d}, J_{\mathrm{P}, \mathrm{C}}=3.9 \mathrm{~Hz}\right), 137.49\left(\mathrm{~d}, J_{\mathrm{P}, \mathrm{C}}=35.4\right.$ $\mathrm{Hz}), 145.45\left(\mathrm{~d}, J_{\mathrm{P}, \mathrm{C}}=21.4 \mathrm{~Hz}\right) ;{ }^{31} \mathrm{P}$ NMR $\left(\mathrm{C}_{6} \mathrm{D}_{6}, 101.3 \mathrm{MHz}\right) \delta-14.81 ; \mathrm{HRMS}(\mathrm{ESI}) \mathrm{m} / \mathrm{z}$ $359.1736\left([\mathrm{M}+\mathrm{O}+\mathrm{Na}]^{+}\right.$, exact mass calcd for $\left.\mathrm{C}_{19} \mathrm{H}_{29} \mathrm{O}_{3} \mathrm{PNa} 359.1747\right)$.

Preparation of 2-(2-bromophenyl)-(4S,5S)-dimethyl-[1,3]dioxolane ${ }^{5}$ :<smiles>O=Cc1ccccc1Br</smiles><smiles>CC(O)C(C)O</smiles>

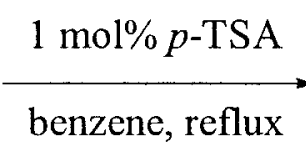<smiles>CC1OC(c2ccccc2Br)O[C@@H]1C</smiles>

The procedure is the same as the above. Starting from 2-bromobenzaldehyde $(0.59 \mathrm{~mL}, 5$ $\mathrm{mmol})$ and $(2 S, 3 S)$-butanediol $(0.5 \mathrm{~g}, 5.58 \mathrm{mmol})$ to get $1.11 \mathrm{~g}(85 \%)$ of 2-(2bromophenyl)-(4S,5S)-dimethyl-[1,3]dioxolane. ${ }^{1} \mathrm{H}$ NMR $\left(\mathrm{CDCl}_{3}, 400 \mathrm{MHz}\right) \delta 7.63$ (dd, $J=7.72,1.74 \mathrm{~Hz}, 1 \mathrm{H}), 7.54(\mathrm{dd} J=7.96,1.12 \mathrm{~Hz}, 1 \mathrm{H}), 7.34(\mathrm{td}, J=7.60,0.96 \mathrm{~Hz}, 1 \mathrm{H})$, $7.20(\mathrm{td}, J=7.84,1.72 \mathrm{~Hz}, 1 \mathrm{H}), 6.24(\mathrm{~s}, 1 \mathrm{H}), 3.95-3.75(\mathrm{~m}, 2 \mathrm{H}), 1.39(\mathrm{~d}, J=5.80 \mathrm{~Hz}$, 
$3 \mathrm{H}), 1.34(\mathrm{~d}, J=5.80 \mathrm{~Hz}, 3 \mathrm{H}) ;{ }^{13} \mathrm{C} \mathrm{NMR}\left(\mathrm{CDCl}_{3}, 100.6 \mathrm{MHz}\right) \delta 17.19,17.51,79.07$, $80.84,101.89,123.35,127.87,128.55,130.90,133.32,137.76$.

Preparation of [2-((4S,5S)-dimethyl-[1,3]dioxolan-2-yl)-phenyl]-phosphonic acid diethyl ester ${ }^{6}$ :

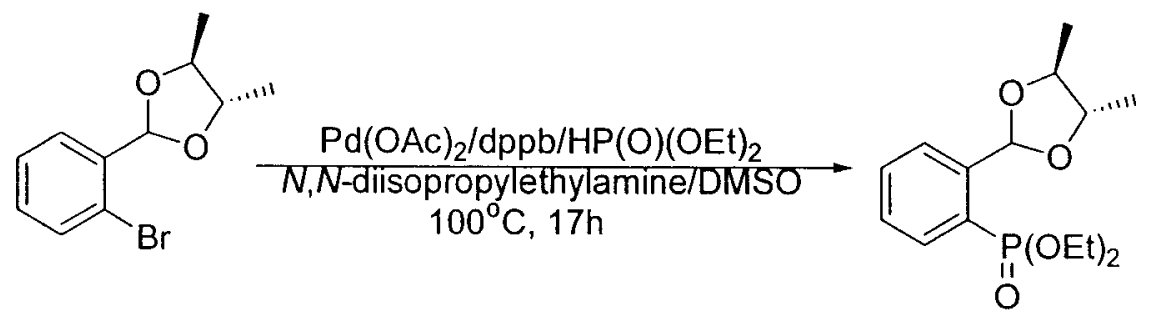

The procedure is the same as the above. Starting from 2-(2-bromophenyl)-(4S,5S)dimethyl-[1,3]dioxolane $(1.765 \mathrm{~g}, 6.87 \mathrm{mmol})$, diethylphosphite $(2.0 \mathrm{~mL}, 15.8 \mathrm{mmol})$, $\mathrm{Pd}(\mathrm{OAc})_{2} \quad(76 \mathrm{mg}, \quad 0.34 \mathrm{mmol}), \quad \mathrm{dppb} \quad(145 \mathrm{mg}, \quad 0.34 \mathrm{mmol})$ and $\mathrm{N}, \mathrm{N}-$ diisopropylethylamine $(4.8 \mathrm{~mL}, 28 \mathrm{mmol})$ to get $1.2 \mathrm{~g} \mathrm{(58 \% )}$ of [2-((4S,5S)-dimethyl[1,3]dioxolan-2-yl)-phenyl]-phosphonic acid diethyl ester and $500 \mathrm{mg}(28 \%)$ of 2-(2bromophenyl)-(4S,5S)-dimethyl-[1,3]dioxolane was recovered. ${ }^{1} \mathrm{H}$ NMR $\left(\mathrm{CDCl}_{3}, 400\right.$ MHz) $\delta 7.90(\mathrm{ddd}, J=14.0,6.50,1.20 \mathrm{~Hz}, 1 \mathrm{H}), 7.76(\mathrm{td}, J=5.50,1.20 \mathrm{~Hz}, 1 \mathrm{H}), 7.54(\mathrm{t}$, $J=7.50 \mathrm{~Hz}, 1 \mathrm{H}), 7.45-7.30(\mathrm{~m}, 1 \mathrm{H}), 6.50(\mathrm{~s}, 1 \mathrm{H}), 4.20-3.90(\mathrm{~m}, 4 \mathrm{H}), 3.85-3.70(\mathrm{~m}, 2 \mathrm{H})$, 1.40-1.15 (m,12H); ${ }^{13} \mathrm{C} \mathrm{NMR}\left(\mathrm{CDCl}_{3}, 100.6 \mathrm{MHz}\right) \delta 16.47,17.14,17.58,62.36,79.11$, 80.63, 99.48, 126.25, $127.90\left(\mathrm{t}, J_{\mathrm{P}, \mathrm{C}}=14.1 \mathrm{~Hz}\right), 128.92\left(\mathrm{~d}, J_{\mathrm{P}, \mathrm{C}}=14.1 \mathrm{~Hz}\right), 132.93$, $133.93\left(\mathrm{~d}, J_{\mathrm{P}, \mathrm{C}}=8.1 \mathrm{~Hz}\right), 142.22\left(\mathrm{~d}, J_{\mathrm{P}, \mathrm{C}}=10.1 \mathrm{~Hz}\right) ;{ }^{31} \mathrm{P} \mathrm{NMR}\left(\mathrm{CDCl}_{3}, 101.3 \mathrm{MHz}\right) \delta$ 19.02; HRMS (ESI) $\mathrm{m} / \mathrm{z} 337.1188\left([\mathrm{M}+\mathrm{Na}]^{+}\right.$, exact mass calcd for $\mathrm{C}_{15} \mathrm{H}_{23} \mathrm{O}_{5} \mathrm{PNa}$ 337.1175).

Preparation of 2-[(4S,5S)-dimethyl-[1,3]dioxolan-2-yl]-phenyl-phosphane ${ }^{7}$ :<smiles>CCOC(=O)c1ccccc1C1OC(C)[C@@H](C)O1</smiles>

$\mathrm{LiAlH}_{4}(2.5$ equiv.) THF, r.t., $0.5 \mathrm{~h}$<smiles>CC1OC(c2ccccc2P)O[C@@H]1C</smiles> 
The procedure is the same as the above. Starting from [2-((4S,5S)-dimethyl[1,3]dioxolan-2-yl)-phenyl]-phosphonic acid diethyl ester (315 $\mathrm{mg}, 1 \mathrm{mmol})$ to get $53 \mathrm{mg}$ (25\%) of 2-[(4S,5S)-dimethyl-[1,3]dioxolan-2-yl]-phenyl-phosphane. ${ }^{1} \mathrm{H}$ NMR $\left(\mathrm{CDCl}_{3}\right.$, $250 \mathrm{MHz}) \delta 7.65-7.45(\mathrm{~m}, 2 \mathrm{H}), 7.40-7.10(\mathrm{~m}, 2 \mathrm{H}), 6.09\left(\mathrm{~d}, J_{\mathrm{P}, \mathrm{H}}=2.0 \mathrm{~Hz}, 1 \mathrm{H}\right), 3.96(\mathrm{~d}$, $\left.J_{\mathrm{P}, \mathrm{H}}=205 \mathrm{~Hz}, 2 \mathrm{H}\right), 3.90-3.70(\mathrm{~m}, 2 \mathrm{H}), 1.35(\mathrm{~d}, J=5.80 \mathrm{~Hz}, 3 \mathrm{H}), 1.27(\mathrm{~d}, J=5.80 \mathrm{~Hz}$, $3 \mathrm{H}) ;{ }^{13} \mathrm{C} \mathrm{NMR}\left(\mathrm{CDCl}_{3}, 62.9 \mathrm{MHz}\right) \delta 17.22,17.78,79.02,81.04,102.23,127.44\left(\mathrm{~d}, J_{\mathrm{P}, \mathrm{C}}\right.$ $=3.4 \mathrm{~Hz}), 128.70,129.16\left(\mathrm{~d}, J_{\mathrm{P}, \mathrm{C}}=3.2 \mathrm{~Hz}\right), 129.35,136.82\left(\mathrm{~d}, J_{\mathrm{P}, \mathrm{C}}=6.3 \mathrm{~Hz}\right), 141.34(\mathrm{~d}$, $\left.J_{\mathrm{P}, \mathrm{C}}=10.7 \mathrm{~Hz}\right) ;{ }^{31} \mathrm{P} \mathrm{NMR}\left(\mathrm{CDCl}_{3}, 101.3 \mathrm{MHz}\right) \delta-126.05 ; \mathrm{HRMS}(\mathrm{ESI}) \mathrm{m} / \mathrm{z} 249.0645$ $\left([\mathrm{M}+\mathrm{O}+\mathrm{Na}]^{+}\right.$, exact mass calcd for $\left.\mathrm{C}_{11} \mathrm{H}_{15} \mathrm{O}_{3} \mathrm{PNa} 249.0651\right)$.

Preparation of 2-[2-((2S,5S)-dimethyl-phospholan-1-yl)-phenyl]-(4S,5S)-dimethyl$[1,3]$ dioxolane $(2)^{8}$ :

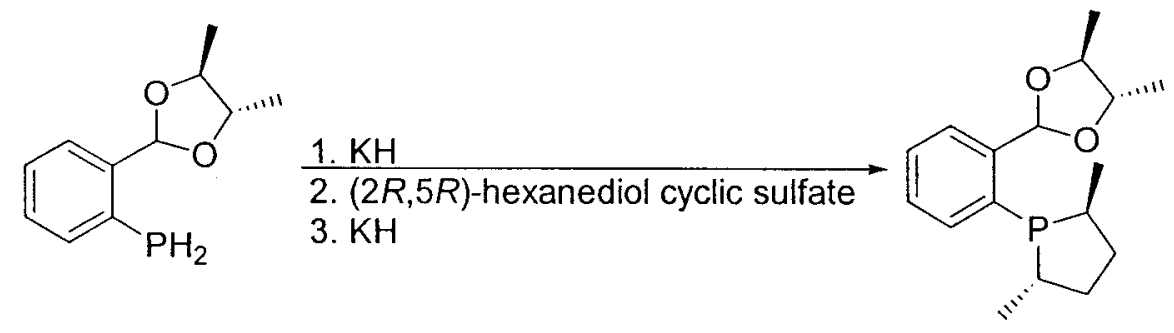

The procedure is the same as the above. Starting from 2-[(4S,5S)-dimethyl-[1,3]dioxolan2-yl]-phenyl-phosphane $(52 \mathrm{mg}, 0.25 \mathrm{mmol})$ and $(2 R, 5 R)$-hexanediol cyclic sulfate (45 $\mathrm{mg}, 0.25 \mathrm{mmol})$ to get $34 \mathrm{mg}(47 \%)$ of 2-[2-((2S,5S)-dimethyl-phospholan-1-yl)-phenyl](4S,5S)-dimethyl-[1,3]dioxolane. ${ }^{1} \mathrm{H}$ NMR $\left(\mathrm{C}_{6} \mathrm{D}_{6}, 250 \mathrm{MHz}\right) \delta 7.97$ (ddd, $J=7.75,3.25$, $1.50 \mathrm{~Hz}, 1 \mathrm{H}), 7.33(\mathrm{dt}, J=7.50,1.50 \mathrm{~Hz}, 1 \mathrm{H}), 7.22(\mathrm{td}, J=7.50,1.25 \mathrm{~Hz}, 1 \mathrm{H}), 7.15-7.05$ (m, 2H), 3.70-3.45 (m, 2H), 2.60-2.40 (m, 1H), 2.40-2.15 (m, 1H), 2.10-1.92 (m, 1H), $1.90-1.72(\mathrm{~m}, 1 \mathrm{H}), 1.53-1.30(\mathrm{~m}, 1 \mathrm{H}), 1.30-1.12(\mathrm{~m}, 4 \mathrm{H}), 1.09(\mathrm{~d}, J=5.75 \mathrm{~Hz}, 3 \mathrm{H}), 1.05$ $(\mathrm{d}, J=6.75 \mathrm{~Hz}, 3 \mathrm{H}), 0.89(\mathrm{dd}, J=9.75,7.0 \mathrm{~Hz}, 3 \mathrm{H}) ;{ }^{13} \mathrm{C} \mathrm{NMR}\left(\mathrm{C}_{6} \mathrm{D}_{6}, 62.9 \mathrm{MHz}\right) \delta$ $16.94\left(\mathrm{~d}, J_{\mathrm{P}, \mathrm{C}}=5.25 \mathrm{~Hz}\right), 17.42,17.89,21.45\left(\mathrm{~d}, J_{\mathrm{P}, \mathrm{C}}=36.5 \mathrm{~Hz}\right), 35.52\left(\mathrm{~d}, J_{\mathrm{P}, \mathrm{C}}=16.25\right.$ $\mathrm{Hz}), 35.71\left(\mathrm{~d}, J_{\mathrm{P}, \mathrm{C}}=6.25 \mathrm{~Hz}\right), 37.05\left(\mathrm{~d}, J_{\mathrm{P}, \mathrm{C}}=9.3 \mathrm{~Hz}\right), 37.50\left(\mathrm{~d}, J_{\mathrm{P}, \mathrm{C}}=10.0 \mathrm{~Hz}\right), 79.08$, $80.80,100.97\left(\mathrm{~d}, J_{\mathrm{P}, \mathrm{C}}=31.1 \mathrm{~Hz}\right), 127.66\left(\mathrm{~d}, J_{\mathrm{P}, \mathrm{C}}=5.7 \mathrm{~Hz}\right), 128.61,129.04,133.14(\mathrm{~d}$, $\left.J_{\mathrm{P}, \mathrm{C}}=3.6 \mathrm{~Hz}\right), 137.15\left(\mathrm{~d}, J_{\mathrm{P}, \mathrm{C}}=28.9 \mathrm{~Hz}\right), 145.60\left(\mathrm{~d}, J_{\mathrm{P}, \mathrm{C}}=20.4 \mathrm{~Hz}\right) ;{ }^{31} \mathrm{P} \mathrm{NMR}\left(\mathrm{C}_{6} \mathrm{D}_{6}\right.$, 
101.3 MHz) $\delta$-5.03; HRMS (ESI) $\mathrm{m} / \mathrm{z} 293.1681\left([\mathrm{M}+\mathrm{H}]^{+}\right.$, exact mass calcd for $\left.\mathrm{C}_{17} \mathrm{H}_{26} \mathrm{O}_{2} \mathrm{P} 293.1665\right)$.

Preparation of 2-(2-bromophenyl)-[1,3]dioxolane ${ }^{5}$ :

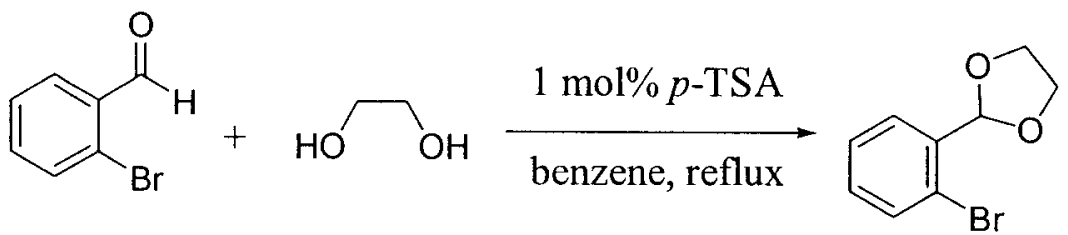

The procedure is the same as the above. Starting from 2-bromobenzaldehyde $(3.7 \mathrm{~g}, 20$ $\mathrm{mmol})$ and ethylene glycol $(1.36 \mathrm{~g}, 22 \mathrm{mmol})$ to get $4.2 \mathrm{~g}(92 \%)$ of 2-(2-bromophenyl)$[1,3]$ dioxolane. ${ }^{1} \mathrm{H}$ NMR $\left(\mathrm{CDCl}_{3}, 250 \mathrm{MHz}\right) \delta 7.47(\mathrm{td}, J=7.75,1.75 \mathrm{~Hz}, 2 \mathrm{H}), 7.22(\mathrm{t}, J$ $=7.5 \mathrm{~Hz}, 1 \mathrm{H}), 7.12(\mathrm{td}, J=5.25,1.55 \mathrm{~Hz}, 1 \mathrm{H}), 5.99(\mathrm{~s}, 1 \mathrm{H}), 4.15-3.90(\mathrm{~m}, 4 \mathrm{H}) ;{ }^{13} \mathrm{C}$ NMR $\left(\mathrm{CDCl}_{3}, 62.9 \mathrm{MHz}\right) \delta 65.68,103.52,127.23,128.66,129.23,136.83,140.66$.

Preparation of (2 -[1,3]dioxolan-2-yl-phenyl)-phosphonic acid diethyl ester ${ }^{6}$ :

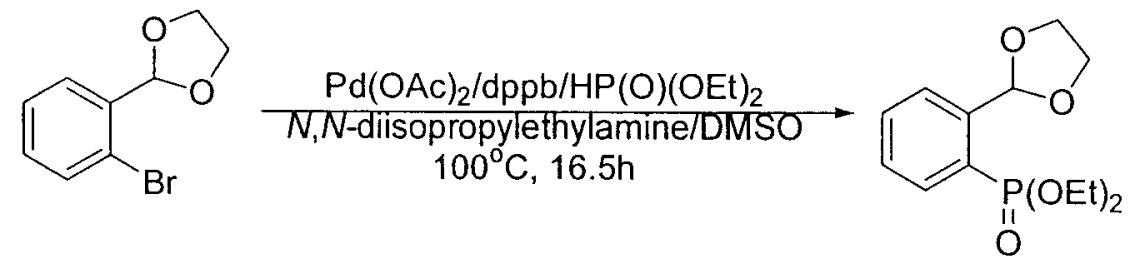

The procedure is the same as the above. Starting from 2-(2-bromophenyl)-[1,3]dioxolane (4.04 g, $17.6 \mathrm{mmol}$ ), diethylphosphite (5.2 mL, $40.5 \mathrm{mmol}), \mathrm{Pd}(\mathrm{OAc})_{2}$ (198 mg, 0.88 mmol), dppb (375 mg, $0.88 \mathrm{mmol})$ and $N, N$-diisopropylethylamine (12.3 $\mathrm{mL}, 70.4 \mathrm{mmol})$ in DMSO (20 mL) to get $2.82 \mathrm{~g}(56 \%)$ of (2 -[1,3]dioxolan-2-yl-phenyl)-phosphonic acid diethyl ester and $1.45 \mathrm{~g}$ (36\%) of 2-(2-bromophenyl)-[1,3]dioxolane was recovered. ${ }^{1} \mathrm{H}$ NMR $\left(\mathrm{CDCl}_{3}, 250 \mathrm{MHz}\right) \delta 7.94(\mathrm{dd}, J=14.0,7.75 \mathrm{~Hz}, 1 \mathrm{H}), 7.76(\mathrm{t}, J=6.50 \mathrm{~Hz}, 1 \mathrm{H})$, $7.56(\mathrm{t}, J=7.50 \mathrm{~Hz}, 1 \mathrm{H}), 7.50-7.35(\mathrm{~m}, 1 \mathrm{H}), 6.40(\mathrm{~s}, 1 \mathrm{H}), 4.30-3.95(\mathrm{~m}, 8 \mathrm{H}), 1.30(\mathrm{t}, J=$ $7.0 \mathrm{~Hz}, 6 \mathrm{H}) ;{ }^{31} \mathrm{P} \mathrm{NMR}\left(\mathrm{CDCl}_{3}, 101.3 \mathrm{MHz}\right) \delta 18.94$.

Preparation of 2 -[1,3]dioxolan-2-yl-phenyl-phosphane ${ }^{7}$ : 


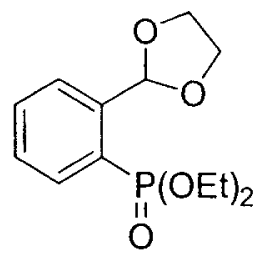

$\mathrm{LiAlH}_{4}(2.5$ equiv.)

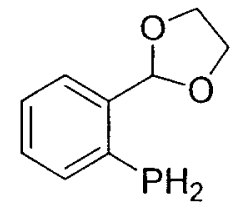

The procedure is the same as the above. Starting from (2 -[1,3]dioxolan-2-yl-phenyl)phosphonic acid diethyl ester (572 $\mathrm{mg}, 2 \mathrm{mmol})$ to get $56 \mathrm{mg}(15.4 \%)$ of 2 [1,3]dioxolan-2-yl-phenyl-phosphane. ' $\mathrm{H} \mathrm{NMR}\left(\mathrm{CDCl}_{3}, 250 \mathrm{MHz}\right) \delta 7.55-7.35(\mathrm{~m}, 2 \mathrm{H})$, 7.30-7.05 (m, 2H), $5.90(\mathrm{~s}, 1 \mathrm{H}), 3.89\left(\mathrm{~d}, J_{\mathrm{P}, \mathrm{H}}=205 \mathrm{~Hz}, 2 \mathrm{H}\right), 4.20-3.90(\mathrm{~m}, 4 \mathrm{H}) ;{ }^{31} \mathrm{P}$ NMR $\left(\mathrm{CDCl}_{3}, 101.3 \mathrm{MHz}\right) \delta-126.04$.

\section{Preparation of 2-[2-((2S,5S)-dimethyl-phospholan-1-yl)-phenyl]-[1,3]dioxolane $(3)^{8}$ :}<smiles>COC(O)c1ccccc1P</smiles>

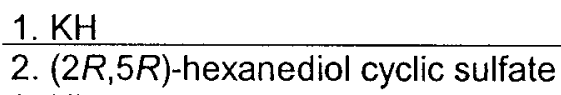
3. $\mathrm{KH}$

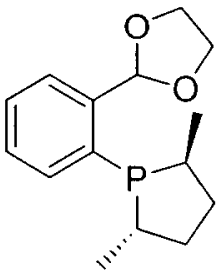

The procedure is the same as the above. Starting from 2 -[1,3]dioxolan-2-yl-phenylphosphane $(56 \mathrm{mg}, 0.31 \mathrm{mmol})$ and $(2 R, 5 R)$-hexanediol cyclic sulfate $(55.4 \mathrm{mg}$, $0.31 \mathrm{mmol})$ to get $37 \mathrm{mg}(45 \%)$ of 2-[2-((2S,5S)-dimethyl-phospholan-1-yl)-phenyl]$[1,3]$ dioxolane. ${ }^{1} \mathrm{H}$ NMR $\left(\mathrm{CDCl}_{3}, 250 \mathrm{MHz}\right) \delta 7.75-7.60(\mathrm{~m}, 1 \mathrm{H}), 7.50-7.40(\mathrm{~m}, 1 \mathrm{H})$, 7.40-7.28 (m, 2H), $6.60\left(\mathrm{~d}, J_{\mathrm{P}, \mathrm{H}}=6.7 \mathrm{~Hz}, 1 \mathrm{H}\right), 4.25-4.00(\mathrm{~m}, 4 \mathrm{H}), 2.80-2.60(\mathrm{~m}, 1 \mathrm{H})$, 2.50-2.20 (m, 2H), 2.15-1.95 (m, 1H), 1.70-1.45 (m, 1H), 1.45-1.25 (m, 4H), $0.76(\mathrm{dd}, \mathrm{J}$ $=10.1,7.25 \mathrm{~Hz}, 3 \mathrm{H}) ;{ }^{13} \mathrm{C} \mathrm{NMR}\left(\mathrm{CDCl}_{3}, 62.9 \mathrm{MHz}\right) \delta 15.99\left(\mathrm{~d}, J_{\mathrm{P}, \mathrm{C}}=1.3 \mathrm{~Hz}\right), 20.84(\mathrm{~d}$, $\left.J_{\mathrm{P}, \mathrm{C}}=35.2 \mathrm{~Hz}\right), 34.80\left(\mathrm{~d}, J_{\mathrm{P}, \mathrm{C}}=7.6 \mathrm{~Hz}\right), 34.96\left(\mathrm{~d}, J_{\mathrm{P}, \mathrm{C}}=5.4 \mathrm{~Hz}\right), 36.39\left(\mathrm{~d}, J_{\mathrm{P}, \mathrm{C}}=2.3 \mathrm{~Hz}\right)$, $36.83\left(\mathrm{~d}, J_{\mathrm{P}, \mathrm{C}}=2.7 \mathrm{~Hz}\right), 65.42\left(\mathrm{~d}, J_{\mathrm{P}, \mathrm{C}}=5.7 \mathrm{~Hz}\right), 101.31\left(\mathrm{~d}, J_{\mathrm{P}, \mathrm{C}}=30.2 \mathrm{~Hz}\right), 126.10(\mathrm{~d}$, $\left.J_{\mathrm{P}, \mathrm{C}}=5.7 \mathrm{~Hz}\right), 128.29,128.68,132.50\left(\mathrm{~d}, J_{\mathrm{P}, \mathrm{C}}=3.5 \mathrm{~Hz}\right), 136.06\left(\mathrm{~d}, J_{\mathrm{P}, \mathrm{C}}=33.9 \mathrm{~Hz}\right)$, $143.08\left(\mathrm{~d}, J_{\mathrm{P}, \mathrm{C}}=20.1 \mathrm{~Hz}\right) ;{ }^{31} \mathrm{P} \mathrm{NMR}\left(\mathrm{CDCl}_{3}, 101.3 \mathrm{MHz}\right) \delta-4.64 ; \mathrm{HRMS}(\mathrm{ESI}) \mathrm{m} / \mathrm{z}$ $303.1126\left([\mathrm{M}+\mathrm{O}+\mathrm{Na}]^{+}\right.$, exact mass calcd for $\left.\mathrm{C}_{15} \mathrm{H}_{21} \mathrm{O}_{3} \mathrm{PNa} 303.1121\right)$.

\section{Preparation of 2-(2-bromophenyl)-(4S,6S)-dimethyl-[1,3]-dioxane : $^{5}$}




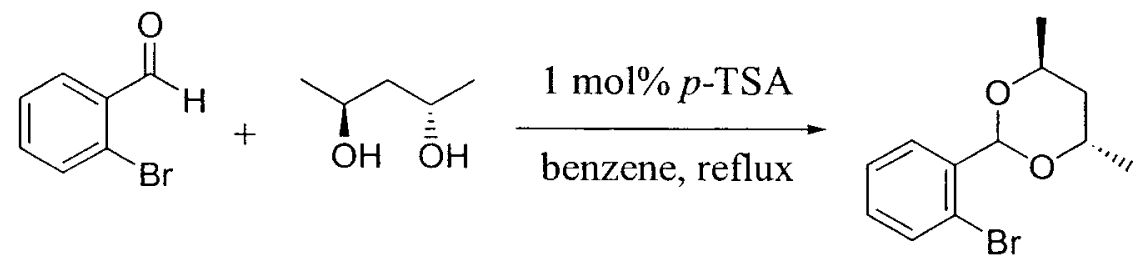

The procedure is the same as the above. Starting from 2-bromobenzaldehyde $(0.80 \mathrm{~g}$, $4.36 \mathrm{mmol} \mathrm{mmol})$ and $(2 S, 4 S)$-pentanediol $(0.5 \mathrm{~g}, 4.8 \mathrm{mmol})$ to get $1.05 \mathrm{~g}(90 \%)$ of 2-(2bromophenyl)-(4S,6S)-dimethyl-[1,3]-dioxane. ${ }^{1} \mathrm{H}$ NMR $\left(\mathrm{CDCl}_{3}, 400 \mathrm{MHz}\right) \delta 7.73(\mathrm{dd}, J$ $=7.96,1.68 \mathrm{~Hz}, 1 \mathrm{H}), 7.51(\mathrm{dd}, J=7.96,0.99 \mathrm{~Hz}, 1 \mathrm{H}), 7.32(\mathrm{td}, J=7.96,0.99 \mathrm{~Hz}, 1 \mathrm{H})$, $7.16(\mathrm{td}, J=7.96,1.68 \mathrm{~Hz}, 1 \mathrm{H}), 6.11(\mathrm{~s}, 1 \mathrm{H}), 4.53-4.40(\mathrm{~m}, 1 \mathrm{H}), 4.28-4.15(\mathrm{~m}, 1 \mathrm{H}), 2.05-$ $1.90(\mathrm{~m}, 1 \mathrm{H}), 1.52(\mathrm{~d}, J=7.0 \mathrm{~Hz}, 3 \mathrm{H}), 1.42(\mathrm{~d}, J=13.6 \mathrm{~Hz}, 1 \mathrm{H}), 1.28(\mathrm{~d}, J=6.4 \mathrm{~Hz}, 3 \mathrm{H})$; ${ }^{13} \mathrm{C} \mathrm{NMR}\left(\mathrm{CDCl}_{3}, 100.6 \mathrm{MHz}\right) \delta 16.94,21.83,36.66,68.33,69.04,93.26,122.38$, 127.48, 128.20, 130.05, 132.47, 137.76 .

Preparation of $[2-((4 S, 6 S)$-dimethyl-[1,3]dioxan-2-yl)phenyl]-phosphonic acid diethyl ester ${ }^{6}$ :
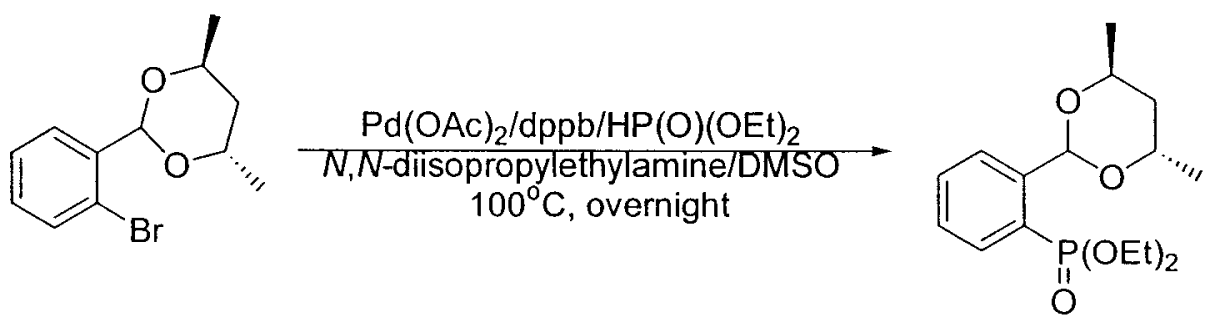

The procedure is the same as the above. Starting from 2-(2-bromophenyl)-(4S,6S)dimethyl-[1,3]-dioxane $(0.79 \mathrm{~g}, 2.9 \mathrm{mmol})$, diethylphosphite $(0.86 \mathrm{~mL}, 6.7 \mathrm{mmol})$, $\mathrm{Pd}(\mathrm{OAc})_{2}$ (33 mg, $0.15 \mathrm{mmol}$ ), dppb (62 mg, $0.15 \mathrm{mmol}$ ) and $N, N$-diisopropylethylamine $(2.0 \mathrm{~mL}, 11.6 \mathrm{mmol})$ in DMSO $(6 \mathrm{~mL})$ to get $0.69 \mathrm{~g}(73 \%)$ of $[2-((4 S, 6 S)$-dimethyl[1,3]dioxan-2-yl)phenyl]-phosphonic acid diethyl ester. ' $\mathrm{H}$ NMR $\left(\mathrm{CDCl}_{3}, 250 \mathrm{MHz}\right) \delta$ 8.00-7.85 (m, 2H), $7.57(\mathrm{t}, J=7.50 \mathrm{~Hz}, 1 \mathrm{H}), 7.45-7.30(\mathrm{~m}, 1 \mathrm{H}), 6.54(\mathrm{~s}, 1 \mathrm{H}), 4.55-4.40$ $(\mathrm{m}, 1 \mathrm{H}), 4.30-3.90(\mathrm{~m}, 5 \mathrm{H}), 2.10-1.90(\mathrm{~m}, 1 \mathrm{H}), 1.50(\mathrm{~d}, J=7.0 \mathrm{~Hz}, 3 \mathrm{H}), 1.42(\mathrm{t}, J=13.5$ $\mathrm{Hz}, 1 \mathrm{H}), 1.35-1.20(\mathrm{~m}, 9 \mathrm{H}) ;{ }^{13} \mathrm{C} \mathrm{NMR}\left(\mathrm{CDCl}_{3}, 100.6 \mathrm{MHz}\right) \delta 16.65\left(\mathrm{t}, J_{\mathrm{P}, \mathrm{C}}=6.5 \mathrm{~Hz}\right)$, $17.63,22.33,37.34,62.46\left(\mathrm{t}, J_{\mathrm{P}, \mathrm{C}}=5.2 \mathrm{~Hz}\right), 68.73,69.50,91.91\left(\mathrm{~d}, J_{\mathrm{P}, \mathrm{C}}=3.9 \mathrm{~Hz}\right), 125.80$ $\left(\mathrm{d}, J_{\mathrm{P}, \mathrm{C}}=181 \mathrm{~Hz}\right), 128.13\left(\mathrm{~d}, J_{\mathrm{P}, \mathrm{C}}=13.6 \mathrm{~Hz}\right), 128.75\left(\mathrm{~d}, J_{\mathrm{P}, \mathrm{C}}=14.1 \mathrm{~Hz}\right), 133.34\left(\mathrm{~d}, J_{\mathrm{P}, \mathrm{C}}=\right.$ 
$2.5 \mathrm{~Hz}), 133.95\left(\mathrm{~d}, J_{\mathrm{P}, \mathrm{C}}=9.1 \mathrm{~Hz}\right), 142.78\left(\mathrm{~d}, J_{\mathrm{P}, \mathrm{C}}=10.1 \mathrm{~Hz}\right) ;{ }^{31} \mathrm{P}$ NMR $\left(\mathrm{CDCl}_{3}, 101.3\right.$ $\mathrm{MHz}) \delta 19.47$.

Preparation of [2-((4S,6S)-dimethyl-[1,3]dioxan-2-yl)phenyl]-phosphane ${ }^{7}$ :

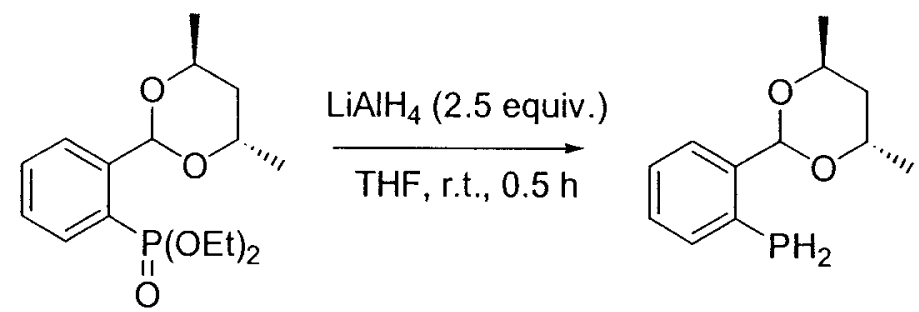

The procedure is the same as the above. Starting from [2 -( $(4 S, 6 S)$-dimethyl-[1,3]dioxan2-yl)phenyl]-phosphonic acid diethyl ester (328 mg, $1 \mathrm{mmol}$ ) to get $73 \mathrm{mg}(32.6 \%)$ of [2 -((4S,6S)-dimethyl-[1,3]dioxan-2-yl)phenyl]-phosphane. ${ }^{1} \mathrm{H}$ NMR $\left(\mathrm{C}_{6} \mathrm{D}_{6}, 400 \mathrm{MHz}\right) \delta$ 8.00-7.85 (m, 1H), $7.30(\mathrm{t}, \mathrm{J}=6.60 \mathrm{~Hz}, 1 \mathrm{H}), 7.10(\mathrm{t}, J=7.40 \mathrm{~Hz}, 1 \mathrm{H}), 6.93(\mathrm{t}, J=7.40$ $\mathrm{Hz}, 1 \mathrm{H}), 6.01(\mathrm{~s}, 1 \mathrm{H}), 4.35-4.15(\mathrm{~m}, 2 \mathrm{H}), 3.90-3.75(\mathrm{~m}, 1 \mathrm{H}), 3.74(\mathrm{~s}, 1 \mathrm{H}), 1.80-1.65(\mathrm{~m}$, $1 \mathrm{H}), 1.18(\mathrm{~d}, J=6.96 \mathrm{~Hz}, 3 \mathrm{H}), 1.09(\mathrm{~d}, J=6.2 \mathrm{~Hz}, 3 \mathrm{H}), 0.86(\mathrm{~d}, J=13.2 \mathrm{~Hz}, 3 \mathrm{H}) ;{ }^{13} \mathrm{C}$ NMR $\left(\mathrm{C}_{6} \mathrm{D}_{6}, 62.9 \mathrm{MHz}\right) \delta 16.95,21.94,36.76,67.87,68.76,93.26\left(\mathrm{~d}, J_{\mathrm{P}, \mathrm{C}}=9.7 \mathrm{~Hz}\right)$, $126.81\left(\mathrm{~d}, J_{\mathrm{P}, \mathrm{C}}=3.4 \mathrm{~Hz}\right), 128.22\left(\mathrm{~d}, J_{\mathrm{P}, \mathrm{C}}=2.0 \mathrm{~Hz}\right), 128.28,128.83,136.21\left(\mathrm{~d}, J_{\mathrm{P}, \mathrm{C}}=4.7\right.$ $\mathrm{Hz}), 143.01\left(\mathrm{~d}, J_{\mathrm{P}, \mathrm{C}}=12.3 \mathrm{~Hz}\right) ;{ }^{31} \mathrm{P}$ NMR $\left(\mathrm{C}_{6} \mathrm{D}_{6}, 101.3 \mathrm{MHz}\right) \delta-112.92$.

Preparation of 2-[2-((2S,5S)-dimethyl-phospholan-1-yl)-phenyl]-(4S,6S)-dimethyl$[1,3]$ dioxane $(4)^{8}$ :

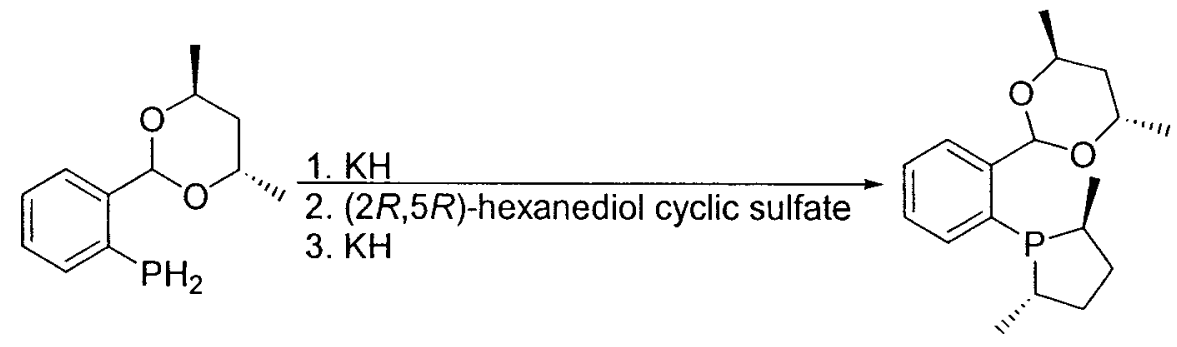

The procedure is the same as the above. Starting from [2 -((4S,6S)-dimethyl-[1,3]dioxan2-yl)phenyl]-phosphane (39 mg, $0.17 \mathrm{mmol})$ and (2R,5R)-hexanediol cyclic sulfate ( 31.3 $\mathrm{mg}, 0.17 \mathrm{mmol})$ to get $20 \mathrm{mg}(38 \%)$ of 2-[2-((2S,5S)-dimethyl-phospholan-1-yl)-phenyl]- 
(4S,6S)-dimethyl-[1,3]dioxane. ${ }^{1} \mathrm{H}$ NMR $\left(\mathrm{C}_{6} \mathrm{D}_{6}, 400 \mathrm{MHz}\right) \delta 8.18$ (ddd, $J=7.8,3.3,1.4$ $\mathrm{Hz}, 1 \mathrm{H}), 7.32(\mathrm{dt}, J=7.5,1.6 \mathrm{~Hz}, 1 \mathrm{H}), 7.22(\mathrm{td}, J=7.5,1.4 \mathrm{~Hz}, 1 \mathrm{H}), 7.11(\mathrm{td}, J=7.5,1.6$ $\mathrm{Hz}, 1 \mathrm{H}), 6.96\left(\mathrm{~d}, J_{\mathrm{P}, \mathrm{H}}=7.4 \mathrm{~Hz}, 1 \mathrm{H}\right), 4.30-4.20(\mathrm{~m}, 1 \mathrm{H}), 4.00-3.90(\mathrm{~m}, 1 \mathrm{H}), 2.60-2.45(\mathrm{~m}$, $1 \mathrm{H}), 2.40-2.25(\mathrm{~m}, 1 \mathrm{H}), 2.10-1.95(\mathrm{~m}, 1 \mathrm{H}), 1.90-1.70(\mathrm{~m}, 2 \mathrm{H}), 1.53-1.40(\mathrm{~m}, 1 \mathrm{H}), 1.39(\mathrm{~d}$, $J=7.0 \mathrm{~Hz}, 3 \mathrm{H}), 1.30-1.15(\mathrm{~m}, 4 \mathrm{H}), 1.12(\mathrm{~d}, J=6.1 \mathrm{~Hz}, 3 \mathrm{H}), 0.95-0.85(\mathrm{~m}, 4 \mathrm{H}) ;{ }^{13} \mathrm{C}$ NMR $\left(\mathrm{C}_{6} \mathrm{D}_{6}, 100.6 \mathrm{MHz}\right) \delta 16.70,17.57\left(\mathrm{~d}, J_{\mathrm{P}, \mathrm{C}}=2.0 \mathrm{~Hz}\right), 20.98\left(\mathrm{~d}, J_{\mathrm{P}, \mathrm{C}}=36.2 \mathrm{~Hz}\right)$, $22.18,35.02\left(\mathrm{~d}, J_{\mathrm{P}, \mathrm{C}}=12.6 \mathrm{~Hz}\right), 35.16\left(\mathrm{~d}, J_{\mathrm{P}, \mathrm{C}}=10.1 \mathrm{~Hz}\right), 36.66\left(\mathrm{~d}, J_{\mathrm{P}, \mathrm{C}}=2.8 \mathrm{~Hz}\right), 37.02$, $37.13\left(\mathrm{~d}, J_{\mathrm{P}, \mathrm{C}}=2.5 \mathrm{~Hz}\right), 67.98,69.14,92.36\left(\mathrm{~d}, J_{\mathrm{P}, \mathrm{C}}=31.2 \mathrm{~Hz}\right), 127.46\left(\mathrm{~d}, J_{\mathrm{P}, \mathrm{C}}=5.4 \mathrm{~Hz}\right)$, $127.85,128.74,132.36\left(\mathrm{~d}, J_{\mathrm{P}, \mathrm{C}}=3.7 \mathrm{~Hz}\right), 135.10\left(\mathrm{~d}, J_{\mathrm{P}, \mathrm{C}}=33.2 \mathrm{~Hz}\right), 146.02\left(\mathrm{~d}, J_{\mathrm{P}, \mathrm{C}}=\right.$ $21.1 \mathrm{~Hz}) ;{ }^{3 !} \mathrm{P}$ NMR $\left(\mathrm{C}_{6} \mathrm{D}_{6}, 101.3 \mathrm{MHz}\right) \delta 8.28$; HRMS (ESI) $\mathrm{m} / \mathrm{z} 345.1588\left([\mathrm{M}+\mathrm{O}+\mathrm{Na}]^{+}\right.$, exact mass calcd for $\mathrm{C}_{18} \mathrm{H}_{27} \mathrm{O}_{3} \mathrm{PNa} 345.1590$ ).

Preparation of 2-[2-((2R,5R)-dimethyl-phospholan-1-yl)-phenyl]-(4S,6S)-dimethyl$[1,3]$ dioxane $(5)^{8}$ :

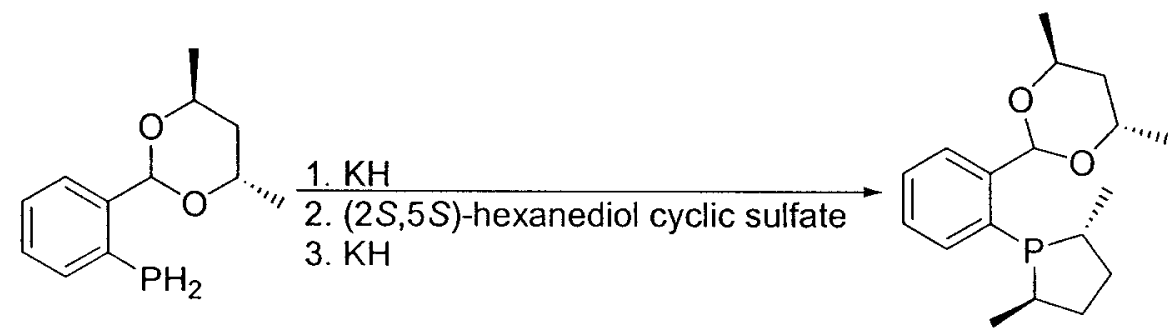

The procedure is the same as the above. Starting from [2 -((4S,6S)-dimethyl-[1,3]dioxan2-yl)phenyl]-phosphane (53 mg, $0.24 \mathrm{mmol})$ and (2S,5S)-hexanediol cyclic sulfate (43.2 $\mathrm{mg}, 0.24 \mathrm{mmol})$ to get $27 \mathrm{mg}(36 \%)$ of 2-[2-((2R,5R)-dimethyl-phospholan-1-yl)-phenyl](4S,6S)-dimethyl-[1,3]dioxane. ${ }^{1} \mathrm{H}$ NMR $\left(\mathrm{C}_{6} \mathrm{D}_{6}, 400 \mathrm{MHz}\right) \delta 8.18$ (ddd, $J=7.8,3.3,1.4$ $\mathrm{Hz}, 1 \mathrm{H}), 7.34(\mathrm{dt}, J=7.5,1.6 \mathrm{~Hz}, 1 \mathrm{H}), 7.22(\mathrm{td}, J=7.5,1.4 \mathrm{~Hz}, 1 \mathrm{H}), 7.11(\mathrm{td}, J=7.5,1.6$ $\mathrm{Hz}, 1 \mathrm{H}), 6.85\left(\mathrm{~d}, J_{\mathrm{P}, \mathrm{H}}=7.2 \mathrm{~Hz}, 1 \mathrm{H}\right), 4.35-4.25(\mathrm{~m}, 1 \mathrm{H}), 4.20-4.05(\mathrm{~m}, 1 \mathrm{H}), 2.60-2.45(\mathrm{~m}$, $1 \mathrm{H}), 2.45-2.30(\mathrm{~m}, 1 \mathrm{H}), 2.10-1.95(\mathrm{~m}, 1 \mathrm{H}), 1.95-1.80(\mathrm{~m}, 1 \mathrm{H}), 1.80-1.70(\mathrm{~m}, 1 \mathrm{H}), 1.55-$ $1.40(\mathrm{~m}, 1 \mathrm{H}), 1.35-1.15(\mathrm{~m}, 7 \mathrm{H}), 1.11(\mathrm{~d}, J=6.2 \mathrm{~Hz}, 3 \mathrm{H}), 0.98-0.85(\mathrm{~m}, 4 \mathrm{H}) ;{ }^{13} \mathrm{C}$ NMR $\left(\mathrm{C}_{6} \mathrm{D}_{6}, 100.6 \mathrm{MHz}\right) \delta 16.86\left(\mathrm{~d}, J_{\mathrm{P}, \mathrm{C}}=2.0 \mathrm{~Hz}\right), 17.14,20.93\left(\mathrm{~d}, J_{\mathrm{P}, \mathrm{C}}=36.2 \mathrm{~Hz}\right), 22.13$, $35.01\left(\mathrm{~d}, J_{\mathrm{P}, \mathrm{C}}=10.4 \mathrm{~Hz}\right), 35.12\left(\mathrm{~d}, J_{\mathrm{P}, \mathrm{C}}=7.3 \mathrm{~Hz}\right), 36.54\left(\mathrm{~d}, J_{\mathrm{P}, \mathrm{C}}=2.9 \mathrm{~Hz}\right), 36.99,37.14$ $\left(\mathrm{d}, J_{\mathrm{P}, \mathrm{C}}=2.4 \mathrm{~Hz}\right), 67.74,68.77,92.26\left(\mathrm{~d}, J_{\mathrm{P}, \mathrm{C}}=29.9 \mathrm{~Hz}\right), 126.99\left(\mathrm{~d}, J_{\mathrm{P}, \mathrm{C}}=5.5 \mathrm{~Hz}\right)$, 
$128.63,132.49\left(\mathrm{~d}, J_{\mathrm{P}, \mathrm{C}}=3.6 \mathrm{~Hz}\right), 135.58\left(\mathrm{~d}, J_{\mathrm{P}, \mathrm{C}}=33.6 \mathrm{~Hz}\right), 145.88\left(\mathrm{~d}, J_{\mathrm{P}, \mathrm{C}}=21.1 \mathrm{~Hz}\right)$;

${ }^{31} \mathrm{P}$ NMR $\left(\mathrm{C}_{6} \mathrm{D}_{6}, 101.3 \mathrm{MHz}\right) \delta 9.42$; HRMS (ESI) $\mathrm{m} / \mathrm{z} 345.1582\left([\mathrm{M}+\mathrm{O}+\mathrm{Na}]^{+}\right.$, exact mass calcd for $\mathrm{C}_{18} \mathrm{H}_{27} \mathrm{O}_{3} \mathrm{PNa} 345.1590$ ).

\section{Asymmetric Hydrovinylation Reactions of Vinylarene Compounds}

\section{General procedure for asymmetric hydrovinylation reaction of Vinylarene} compounds using [(allyl) $\mathrm{NiBr}]_{2}$ and ligands in the presence of $\mathrm{NaBARF}$ in $\mathrm{CH}_{2} \mathrm{Cl}_{2}$ :

To a solution of $[(\text { allyl }) \mathrm{NiBr}]_{2}$ in $\mathrm{CH}_{2} \mathrm{Cl}_{2}(1.5 \mathrm{~mL})$ at room temperature was added a solution of ligand in $\mathrm{CH}_{2} \mathrm{Cl}_{2}(1.5 \mathrm{~mL})$ in drybox. The resulting solution was added to a suspension of $\mathrm{NaBARF}$ in $\mathrm{CH}_{2} \mathrm{Cl}_{2}(1 \mathrm{~mL})$. After stirring at room temperature for $1.5 \mathrm{~h}$, the mixture was filtered through a small plug of celite and the precipitate was rinsed with $\mathrm{CH}_{2} \mathrm{Cl}_{2}(1 \mathrm{~mL})$. The filtrate was collected in a Schlenk flask and was taken out of drybox. The catalyst solution was cooled to the desigated temperature in the table. Under one atmosphere of ethylene, the solution of vinylarene compounds in $\mathrm{CH}_{2} \mathrm{Cl}_{2}(3 \mathrm{~mL})$ was added dropwise to the catalyst solution. After stirring for $2 \mathrm{~h}$ at this temperature, the mixture was quenched with half-saturated aqueous $\mathrm{NH}_{4} \mathrm{Cl}$ solution and extracted three times with $10 \mathrm{~mL}$ portions of $\mathrm{CH}_{2} \mathrm{Cl}_{2}$. The combined organic layers were dried over anhydrous $\mathrm{MgSO}_{4}$ and passed through a small plug of silica gel. The filtrate was concentrated to afford the crude products which were analyzed by GC. The enantiomeric excess of the alkene products were determined by HPLC on Chiracel OJ column (hexane/isopropanol system).

Asymmetric hydrovinylation reaction of 4-isobutylstyrene using [(allyl) $\mathrm{NiBr}_{2}$, ligand in the presence of $\mathrm{NaBARF}$ in $\mathrm{CH}_{2} \mathrm{Cl}_{2}$ :

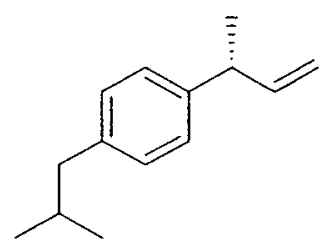


1-Isobutyl-4-[(R)-1-methylallyl]benzene: catalyst amount: $0.35 \mathrm{~mol} \% ;[\alpha]_{\mathrm{D}}=-7.63$ (91\%ee) (c $\left.2.19 \mathrm{CHCl}_{3}\right)\left[\right.$ lit.: $[\alpha]_{\mathrm{D}}=-5.84(83 \%$ ee, neat $\left.),{ }^{9}-6.80\left(\text { c } 2.09 \mathrm{CHCl}_{3}, 74 \% \text { ee }\right)^{10}\right]$; ${ }^{1} \mathrm{H}$ NMR $\left(400 \mathrm{MHz}, \mathrm{CDCl}_{3}\right) \delta 7.10(\mathrm{~d}, J=6.0 \mathrm{~Hz}, 2 \mathrm{H}), 7.05(\mathrm{~d}, J=6.0 \mathrm{~Hz}, 2 \mathrm{H}), 6.05-$ $5.95(\mathrm{~m}, 1 \mathrm{H}), 5.05-4.95(\mathrm{~m}, 2 \mathrm{H}), 3.50-3.35(\mathrm{~m}, 1 \mathrm{H}), 2.42(\mathrm{~d}, J=7.2 \mathrm{~Hz}, 2 \mathrm{H}), 1.90-1.75$ (m, 1H), $1.33(\mathrm{~d}, J=7.0 \mathrm{~Hz}, 3 \mathrm{H}), 0.88(\mathrm{~d}, J=6.6 \mathrm{~Hz}, 6 \mathrm{H}) ; \mathrm{GC}$ conditions: $1.0 \mathrm{~mL}$ helium $/ \mathrm{min}, 10 \mathrm{~min}$ at $100^{\circ} \mathrm{C}, 4^{\circ} \mathrm{C} / \mathrm{min}, 10 \mathrm{~min}$ at $200^{\circ} \mathrm{C}$; HPLC conditions: $100 \%$ hexane, $0.25 \mathrm{~mL} / \mathrm{min}$, retention time $(\mathrm{min}): 21.57(R), 24.08(S)$.

\begin{tabular}{|c|c|c|c|c|c|}
\hline Ligand & Temp. $\left({ }^{\circ} \mathrm{C}\right)$ & Conv. $(\%)$ & Regioselectivity (\%) & Ee (\%) & Configuration \\
\hline $\mathbf{1 a}$ & -55 & 99.9 & 99.6 & 91 & $R$ \\
\hline $\mathbf{1 a}$ & -70 & 100 & 97.5 & 88 & $R$ \\
\hline $\mathbf{1 a}$ & -40 & 100 & 99 & 90 & $R$ \\
\hline $\mathbf{1 b}$ & -55 & 83.4 & 100 & 88 & $R$ \\
\hline $\mathbf{2}$ & -55 & 99.2 & 89.8 & 71 & $R$ \\
\hline $\mathbf{3}$ & -55 & 100 & 100 & 85 & $R$ \\
\hline $\mathbf{4}$ & -55 & 100 & 100 & 85 & $R$ \\
\hline $\mathbf{5}$ & -55 & 100 & 100 & 90 & $S$ \\
\hline
\end{tabular}

Asymmetric hydrovinylation reaction of 4-bromostyrene using $\left[\left(\right.\right.$ allyl) $\mathrm{NiBr}_{2}$, ligand $1 \mathrm{a}$ in the presence of $\mathrm{NaBARF}$ in $\mathrm{CH}_{2} \mathrm{Cl}_{2}$ :<smiles>C=CC(C)c1ccc(Br)cc1</smiles>

1-Bromo-4-[(R)-1-methylallyl]benzene: catalyst amount: 0.35 mol\%; reaction temperature: $-55^{\circ} \mathrm{C}$, conversion: $100 \%$, regioselectivity: $100 \%, 71 \%$ ee, configuration: $R$; $[\alpha]_{\mathrm{D}}=-8.65\left(\right.$ c $\left.3.07 \mathrm{CHCl}_{3}\right)\left(\right.$ lit. $\left.^{10}:[\alpha]_{\mathrm{D}}=+9.9\left(89 \% \mathrm{ee}, \mathrm{c} 7.02 \mathrm{CHCl}_{3}\right)\right) ;{ }^{1} \mathrm{H}$ NMR $(400$ $\left.\mathrm{MHz}, \mathrm{CDCl}_{3}\right) \delta 7.39(\mathrm{~d}, J=9.2 \mathrm{~Hz}, 2 \mathrm{H}), 7.07(\mathrm{~d}, J=9.2 \mathrm{~Hz}, 2 \mathrm{H}), 6.00-5.90(\mathrm{~m}, 1 \mathrm{H})$, 5.05-4.95 (m, 2H), 3.45-3.35 (m, 1H), $1.32(\mathrm{~d}, J=7.0 \mathrm{~Hz}, 3 \mathrm{H})$; GC conditions: $1.0 \mathrm{~mL}$ helium $/ \mathrm{min}, 10 \mathrm{~min}$ at $100^{\circ} \mathrm{C}, 4^{\circ} \mathrm{C} / \mathrm{min}, 25 \mathrm{~min}$ at $200^{\circ} \mathrm{C}$; HPLC conditions: $100 \%$ hexane, $0.30 \mathrm{~mL} / \mathrm{min}$, retention time (min): $21.57(S), 27.70(R)$. 

1a in the presence of $\mathrm{NaBARF}$ in $\mathrm{CH}_{2} \mathrm{Cl}_{2}$ :

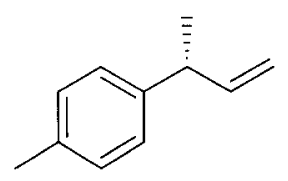

1-Methyl-4- $[(R)-1-$ methylallyl]benzene: catalyst amount: $0.35 \mathrm{~mol} \%$; reaction temperature: $-55^{\circ} \mathrm{C}$, conversion: $100 \%$, regioselectivity: $100 \%, 87 \%$ ee, configuration: $R$; $[\alpha]_{\mathrm{D}}=-9.11$ (c $\left.2.13 \mathrm{CHCl}_{3}\right)\left(\mathrm{lit}^{9}:[\alpha]_{\mathrm{D}}=+6.78(83 \% \mathrm{ee}\right.$, neat $\left.)\right) ;{ }^{1} \mathrm{H}$ NMR $(250 \mathrm{MHz}$, $\left.\mathrm{CDCl}_{3}\right) \delta 7.10(\mathrm{~s}, 4 \mathrm{H}), 6.10-5.90(\mathrm{~m}, 1 \mathrm{H}), 5.10-4.95(\mathrm{~m}, 2 \mathrm{H}), 3.50-3.35(\mathrm{~m}, 1 \mathrm{H}), 2.31(\mathrm{~s}$, $3 \mathrm{H}), 1.32(\mathrm{~d}, \mathrm{~J}=7.0 \mathrm{~Hz}, 3 \mathrm{H})$; $\mathrm{GC}$ conditions: $1.0 \mathrm{~mL}$ helium $/ \mathrm{min}, 5 \mathrm{~min}$ at $80^{\circ} \mathrm{C}$, $4^{\circ} \mathrm{C} / \mathrm{min}, 15 \mathrm{~min}$ at $180^{\circ} \mathrm{C}$; HPLC conditions: $100 \%$ hexane, $0.30 \mathrm{~mL} / \mathrm{min}$, retention time (min): $23.97(S), 25.03(R)$.

Asymmetric hydrovinylation reaction of styrene using [(allyl)NiBr $]_{2}$, ligand $1 \mathrm{a}$ in the presence of $\mathrm{NaBARF}$ in $\mathrm{CH}_{2} \mathrm{Cl}_{2}$ :

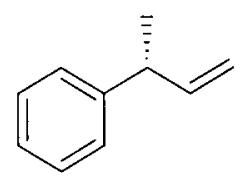

[( $R)$-1-methylallyl]benzene: catalyst amount: $0.35 \mathrm{~mol} \%$; reaction temperature: $-55^{\circ} \mathrm{C}$, conversion: $100 \%$, regioselectivity: $99.7 \%, 88 \%$ ee, configuration: $R ;[\alpha]_{\mathrm{D}}=-4.90(\mathrm{c} 2.06$ $\left.\mathrm{CHCl}_{3}\right)\left(\right.$ lit. ${ }^{9}{ }^{11}:[\alpha]_{\mathrm{D}}=-5.91$ (neat)); ${ }^{1} \mathrm{H} \mathrm{NMR}\left(400 \mathrm{MHz}, \mathrm{CDCl}_{3}\right) \delta 7.35-7.25(\mathrm{~m}, 2 \mathrm{H})$, 7.25-7.10 (m, 3H), 6.15-5.90 (m, 1H), 5.10-4.95 (m, 2H), 3.50-3.40 (m, 1H), $1.35(\mathrm{~d}, \mathrm{~J}=$ $7.0 \mathrm{~Hz}, 3 \mathrm{H}$ ); GC conditions: $1.0 \mathrm{~mL}$ helium $/ \mathrm{min}, 5 \mathrm{~min}$ at $80^{\circ} \mathrm{C}, 4^{\circ} \mathrm{C} / \mathrm{min}, 15 \mathrm{~min}$ at $180^{\circ} \mathrm{C}$; HPLC conditions: $100 \%$ hexane, $0.30 \mathrm{~mL} / \mathrm{min}$, retention time (min): $19.84(R)$, $22.62(S)$.

Asymmetric hydrovinylation reaction of 4-methoxystyrene using $\left[\left(\right.\right.$ allyl) $\mathrm{NiBr}_{2}$, ligand $1 \mathrm{a}$ in the presence of $\mathrm{NaBARF}$ in $\mathrm{CH}_{2} \mathrm{Cl}_{2}$ : 


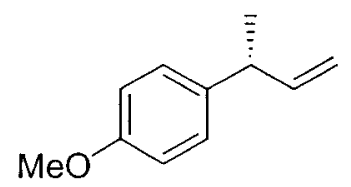

1-Methoxy-4-[(R)-1-methylallyl]benzene: catalyst amount:0.35 mol\%; reaction temperature: $-55^{\circ} \mathrm{C}$, conversion: $80.2 \%$, regioselectivity: $100 \%, 73 \%$ ee, configuration: $R$; GC conditions: $1.0 \mathrm{~mL}$ helium $/ \mathrm{min}, 15 \mathrm{~min}$ at $120^{\circ} \mathrm{C}, 4^{\circ} \mathrm{C} / \mathrm{min}, 30 \mathrm{~min}$ at $220^{\circ} \mathrm{C}$; $\mathrm{HPLC}$ conditions: hexane:isopropanol=95:5, $0.50 \mathrm{~mL} / \mathrm{min}$, retention time $(\mathrm{min}): 26.37(R)$, $27.90(S)$.

Asymmetric hydrovinylation reaction of 6-methoxy-2-vinylnaphthalene using [(allyl) $\mathrm{NiBr}]_{2}$, ligand $1 \mathrm{a}$ in the presence of $\mathrm{NaBARF}$ in $\mathrm{CH}_{2} \mathrm{Cl}_{2}$ :

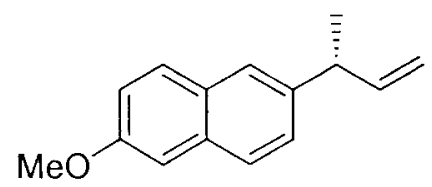

2-Methoxy-6-[(R)-1-methylallyl]naphthalene: catalyst amount:1.40 mol\%; reaction temperature: $-55^{\circ} \mathrm{C}$, conversion: $72.8 \%$, regioselectivity: $100 \%, 86 \%$ ee, configuration: $R$; GC conditions: $1.0 \mathrm{~mL}$ helium $/ \mathrm{min}, 15 \mathrm{~min}$ at $150^{\circ} \mathrm{C}, 4^{\circ} \mathrm{C} / \mathrm{min}, 15 \mathrm{~min}$ at $250^{\circ} \mathrm{C}$; HPLC conditions: hexane:isopropanol $=95: 5,0.50 \mathrm{~mL} / \mathrm{min}$, retention time $(\mathrm{min}): 29.74(R)$, $33.10(S)$.

Asymmetric hydrovinylation reaction of 2-vinylnaphthalene using $[(\text { allyl }) \mathrm{NiBr}]_{2}$, ligand $1 \mathrm{a}$ in the presence of $\mathrm{NaBARF}$ in $\mathrm{CH}_{2} \mathrm{Cl}_{2}$ :

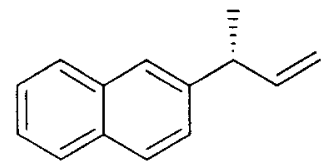

2-[(R)-1-methylallyl]naphthalene: catalyst amount:0.70 mol\%; reaction temperature: $55^{\circ} \mathrm{C}$, conversion: $21 \%$, regioselectivity: $100 \%, 86 \%$ ee, configuration: $R$; GC conditions: $1.0 \mathrm{~mL}$ Helium $/ \mathrm{min}, 5 \mathrm{~min}$ at $130^{\circ} \mathrm{C}, 4^{\circ} \mathrm{C} / \mathrm{min}, 15 \mathrm{~min}$ at $230^{\circ} \mathrm{C} ; \mathrm{HPLC}$ conditions: $100 \%$ hexane, $0.50 \mathrm{~mL} / \mathrm{min}$, retention time $(\mathrm{min}): 36.36(R), 39.58(S)$. 


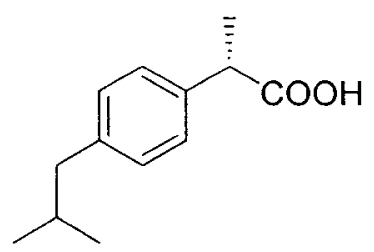

1-Isobutyl-4-[(R)-1-methylallyl]benzene (92 $\mathrm{mg}, 0.49 \mathrm{mmol})$ was dissolved in a mixture of $\mathrm{CH}_{2} \mathrm{Cl}_{2}$ and $\mathrm{CH}_{3} \mathrm{OH}(1: 1,8 \mathrm{~mL})$ and cooled to $-78^{\circ} \mathrm{C}$. Ozone was passed through the solution until it became blue. At that time ozone flow was stopped and nitrogen was bubbled for about $20 \mathrm{~min}$ to expel all the dissolved ozone from the solution. Excess of $\mathrm{Me}_{2} \mathrm{~S}(1 \mathrm{~mL})$ was added and the solution was warmed to room temperature and stirred for $30 \mathrm{~min}$. Excess of water was added and the mixture was extracted with diethyl ether. The combined organic layers were washed with brine and dried over anhydrous $\mathrm{MgSO}_{4}$. The solvent was removed under reduced pressure to get the crude aldehyde which was used for the next step without purification.

To the solution of the crude aldehyde in diethyl ether $(2 \mathrm{~mL})$ was added 2-methyl-2butene $(2 \mathrm{~mL})$ and cooled to $0^{\circ} \mathrm{C}$. Sodium chlorite $(93 \mathrm{mg}, 80 \%, 0.82 \mathrm{mmol})$ which has been powered well was added to the resulting mixture at $0^{\circ} \mathrm{C}$ and stirred vigorously. The reaction mixture was allowed to warm to room temperature for 10 minutes. $5 \mathrm{~mL}$ of water was added and stirred for 3 mintues, then $3 \mathrm{~mL}$ of $2 \mathrm{~N} \mathrm{HCl}$ was added and stirred for additional 5 minutes. The reaction mixture was extracted with diethyl ether. The combined extracts were washed with brine, dried over anhydrous $\mathrm{MgSO}_{4}$ and concentrated to get $95 \mathrm{mg}$ of crude (S)-(+)-ibuprofen. ${ }^{1} \mathrm{H}$ NMR $\left(\mathrm{CDCl}_{3}, 400 \mathrm{MHz}\right) \delta 7.20$ $(\mathrm{d}, J=7.80 \mathrm{~Hz}, 2 \mathrm{H}), 7.08(\mathrm{~d}, J=7.80 \mathrm{~Hz}, 2 \mathrm{H}), 3.69(\mathrm{q}, J=7.20 \mathrm{~Hz}, 1 \mathrm{H}), 2.43(\mathrm{~d}, J=$ $7.20 \mathrm{~Hz}, 2 \mathrm{H}), 1.90-1.75(\mathrm{~m}, 1 \mathrm{H}), 1.48(\mathrm{~d}, J=7.20 \mathrm{~Hz}, 3 \mathrm{H}), 0.88(\mathrm{~d}, J=6.6 \mathrm{~Hz}, 6 \mathrm{H})$.

Determination of enantiomeric excess of ibuprofen: To the solution of ibuprofen (1 mg) in $\mathrm{CH}_{2} \mathrm{Cl}_{2}(1 \mathrm{~mL})$ was added $40 \mathrm{uL}$ of esterification solution which was prepared by mixing (-)-menthol (350 mg), DCC (120 mg), DMAP (6 mg), $25 \mathrm{uL}$ of $1 \mathrm{M} \mathrm{HCl}$ and $\mathrm{CH}_{2} \mathrm{Cl}_{2}(1 \mathrm{~mL})$. The mixture was shaken for about $15 \mathrm{~min}$ and analyzed by Chirasil-LVal on WCOT fused silica ( $25 \mathrm{~m} \times 0.25 \mathrm{~mm}, 0.12 \mu \mathrm{m}$ film thickness) capillary GC 
column to give $89 \%$ ee. GC conditions: $1.0 \mathrm{~mL}$ helium $/ \mathrm{min}, 20 \mathrm{~min}$ at $150^{\circ} \mathrm{C}, 0.5^{\circ} \mathrm{C} / \mathrm{min}$, $30 \mathrm{~min}$ at $180^{\circ} \mathrm{C}$, retention time (min): $23.568(S), 24.307(R)$.

\section{Asymmetric hydrovinylation reaction of 4-isobutylstyrene using $0.035 \mathrm{~mol} \%$ of [(allyl)NiBr $]_{2}$ :}

To a solution of $[(\text { allyl }) \mathrm{NiBr}]_{2}(2.5 \mathrm{mg}, 0.007 \mathrm{~mol})$ in $\mathrm{CH}_{2} \mathrm{Cl}_{2}(1 \mathrm{~mL})$ at room temperature was added a solution of ligand $1 \mathrm{a}(4.3 \mathrm{mg}, 0.014 \mathrm{mmol})$ in $\mathrm{CH}_{2} \mathrm{Cl}_{2}(1 \mathrm{~mL})$ in drybox. The resulting solution was added to a suspension of NaBARF $(12.9 \mathrm{mg}, 0.0146 \mathrm{mmol})$ in $\mathrm{CH}_{2} \mathrm{Cl}_{2}(1 \mathrm{~mL}) .1 \mathrm{~mL}$ of $\mathrm{CH}_{2} \mathrm{Cl}_{2}$ was used to rinse the vial and combined with the above mixture and the resulting mixture was stirred for $1.5 \mathrm{~h}$ at room temperature. The above solution $(0.4 \mathrm{~mL})$ was transferred into a dry Schlenk flask and taken out of drybox. The catalyst solution was cooled to $-55^{\circ} \mathrm{C}$ and added dropwise the solution of 4isobutylstyrene $(320 \mathrm{mg}, 2 \mathrm{mmol})$ in $\mathrm{CH}_{2} \mathrm{Cl}_{2}(1 \mathrm{~mL})$ under 1 atm of ethylene. After stirring at $-55^{\circ} \mathrm{C}$ for $4 \mathrm{~h}$, the mixture was quenched with half-saturated aqueous $\mathrm{NH}_{4} \mathrm{Cl}$ solution and extracted three times with $10 \mathrm{~mL}$ portions of $\mathrm{CH}_{2} \mathrm{Cl}_{2}$. The combined organic layers were dried over anhydrous $\mathrm{MgSO}_{4}$ and passed through a small plug of silica gel. The filtrate was concentrated to afford the crude products which were analyzed by GC to get $86 \%$ conversion and $100 \%$ regioselectivity. The enantiomeric excess of product was determined by HPLC on Chiracel OJ column to be $76 \%$.

\section{References:}

1. Corey, E. J.; Semmelhack, M. F. J. Am. Chem. Soc. 1967, 89, 2755.

2. Brookhart, M.; Grant, B.; Volpe, A. F. Organometallics 1992, 11, 3920.

3. Nugent, W. A.; McKinney, R. J. J. Org. Chem. 1985, 50, 5370.

4. Burk, M. J.; Elaster, J. E.; Nugent, W. A.; Harlow, R. L. J. Am. Chem. Soc. 1993, 115, 10125 .

5. Harada, T.; Nakamura, T.; Kinugasa, M.; Oku, A. J. Org. Chem. 1999, 64, 7594.

6. Uozumi, Y.; Tanahashi, A.; Lee, S.-Y.; Hayashi, T. J. Org. Chem. 1993, 58, 1945.

7. Reduction of diethoxyphosphoryl compounds: (a) Kalbitz, J.; Leibring, E.; Schmidt, H. Z. Anorg. Allg. Chem. 1994, 620, 2041; (b) Kyba, E. P.; Liu, S. T.; Harris, R. L. Organometallics 1983, 2, 1877.

8. Nandi, M.; Jin, J.; RajanBabu, T. V. J. Am. Chem. Soc. 1999, 121, 9899. 
9. Hayashi, T.; Konishi, M.; Fukushima, M.; Kanehira, K.; Hioki, T.; Kumada, M. J. Org. Chem. 1983, 48, 2195.

10. Park, H. and RajanBabu, T. V. J. Am. Chem. Soc. 2002, 124, 734.

11. Hayashi, T.; Konishi, M.; Fukushima, M.; Mise, T.; Kagotani, M.; Tajika, M.; Kumada, M. J. Am. Chem. Soc. 1982, 104, 180. 


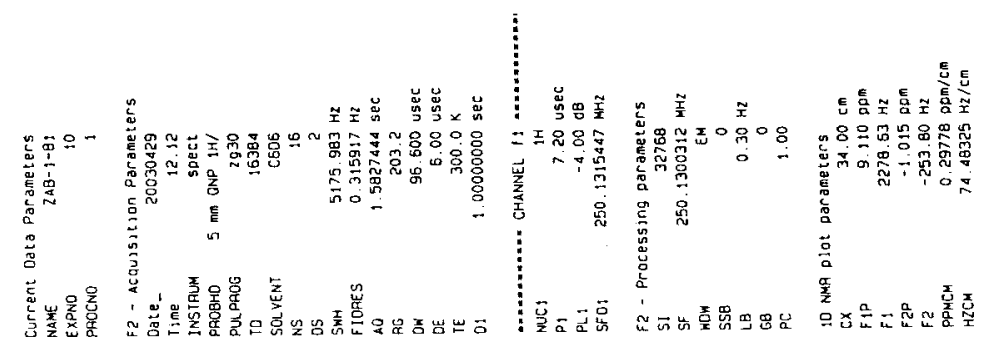

SELCO 0

;8106. 07

:8006.0

उBtE0.17

JEODO $t$

उ9590.1 17

$=0090^{\circ} \mathrm{T}-\mathrm{fl}$

5980 工

C $\angle 9 \angle 1$ : -

$9161: 1 /$

$\angle 6667: 1-$

$\angle \angle 652 \cdot 1-1$

उLPEC 1-

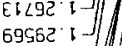

EEOEO [I]

625ta I -

$9[\angle 2 B$ I

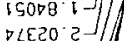

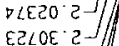

$158<0,5]$

$00205 \cdot 5-$

[8609 2$]$



8ट65S $\mathrm{E}$

E99 LS E-

L ᄃE85. $\varepsilon-$

ट्2629 E-।

E5IG9 $\varepsilon-$

$995 \angle 9 \cdot \varepsilon-$

$98189 \cdot \varepsilon-$

$99669 \cdot E-$
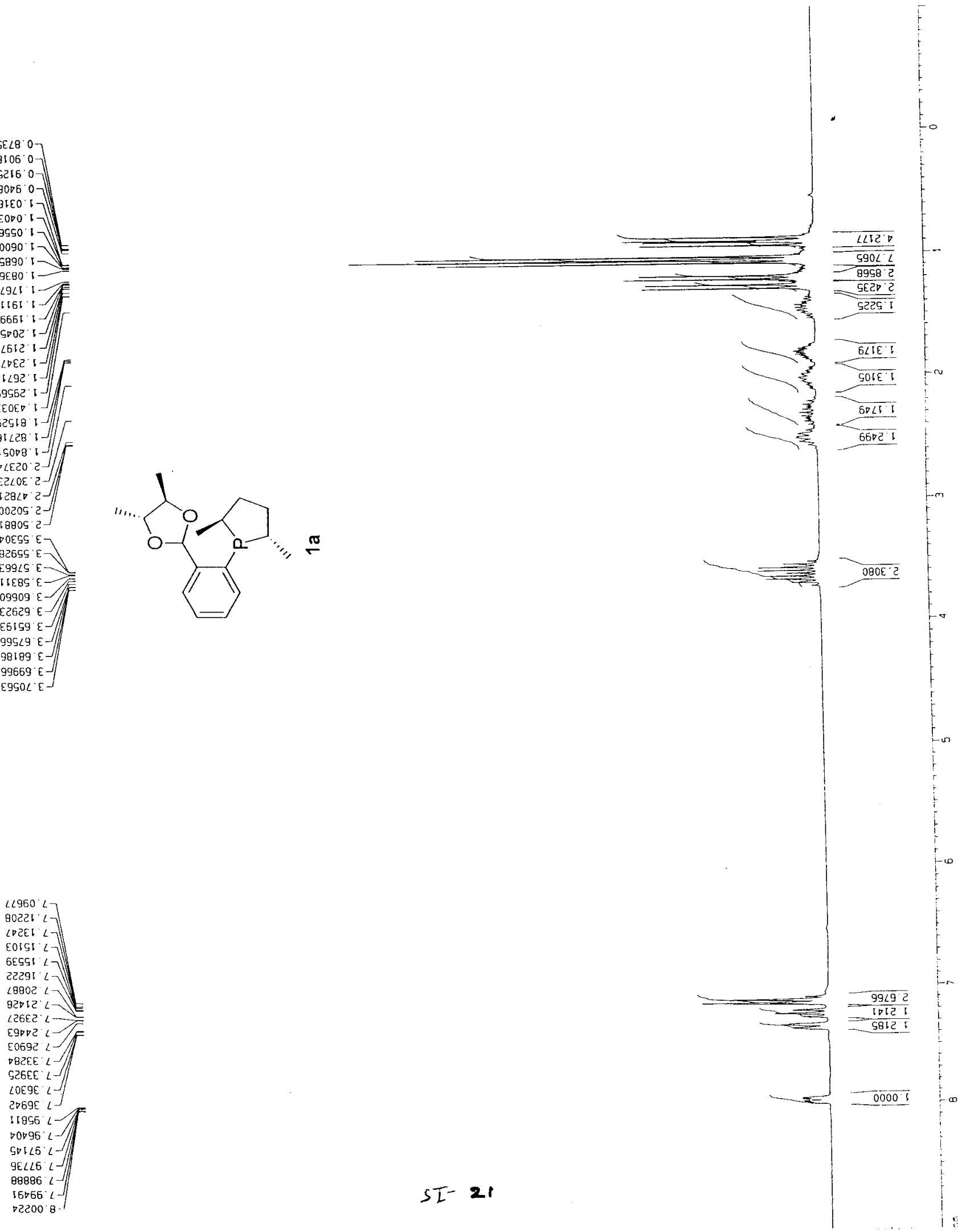

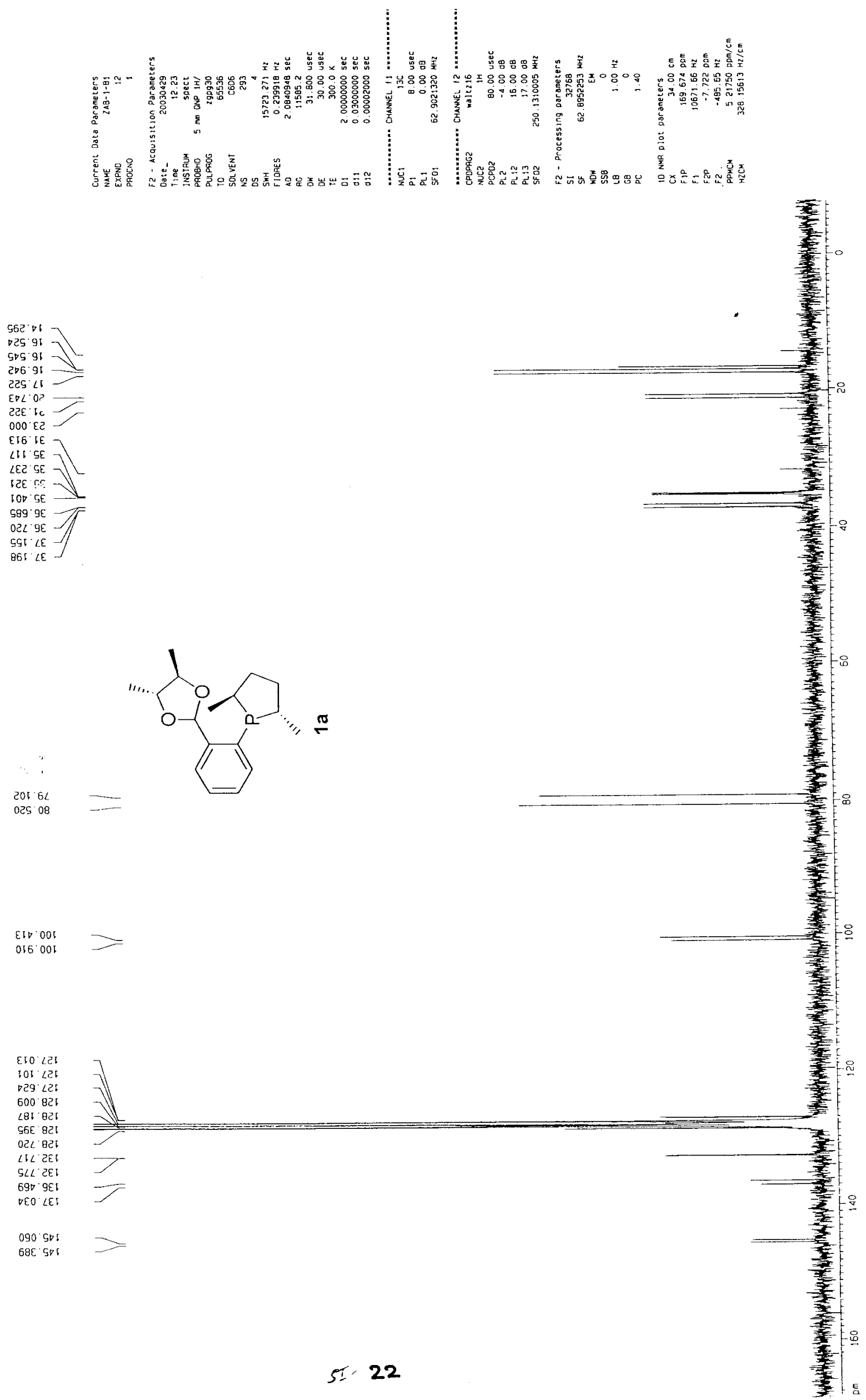


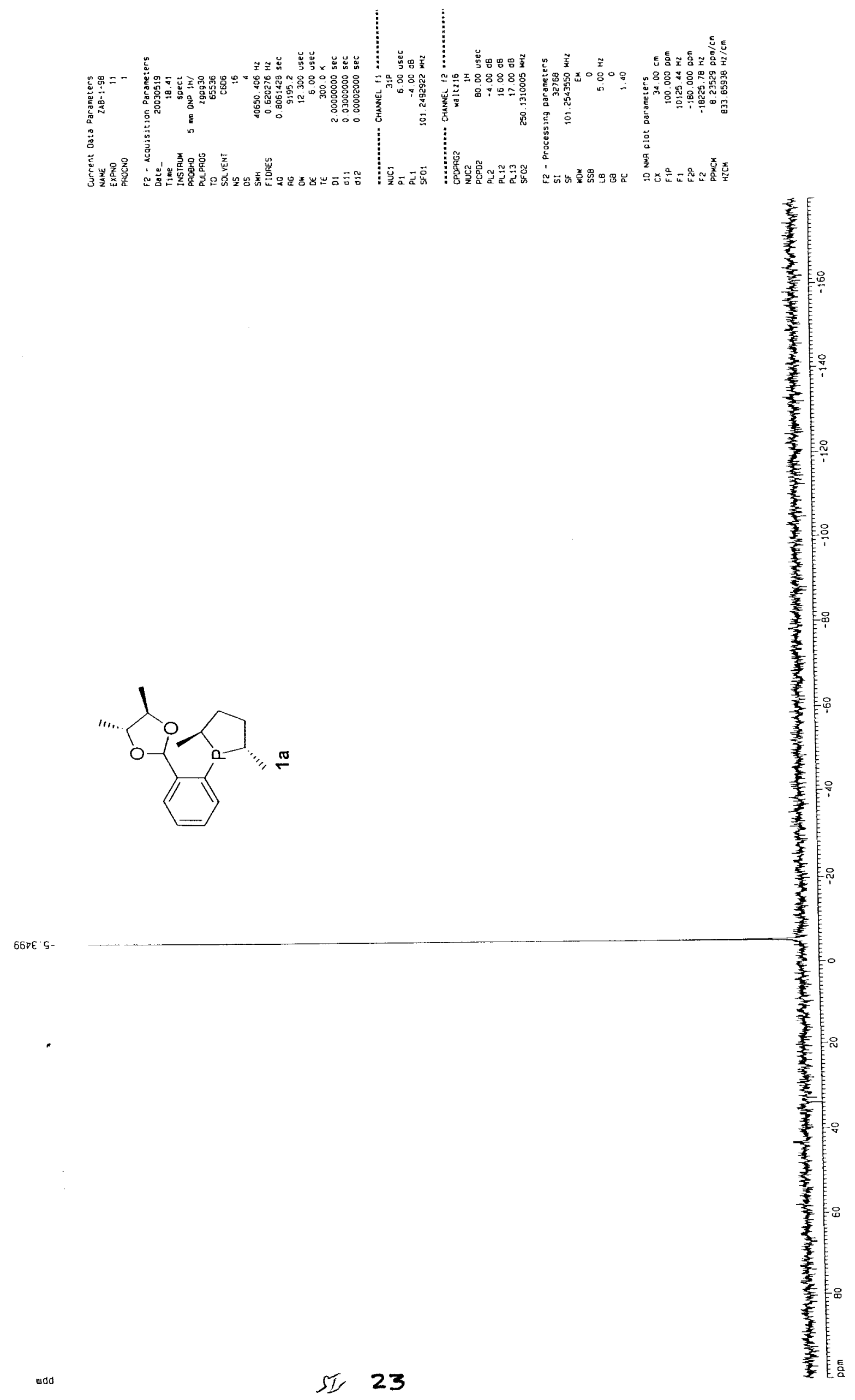



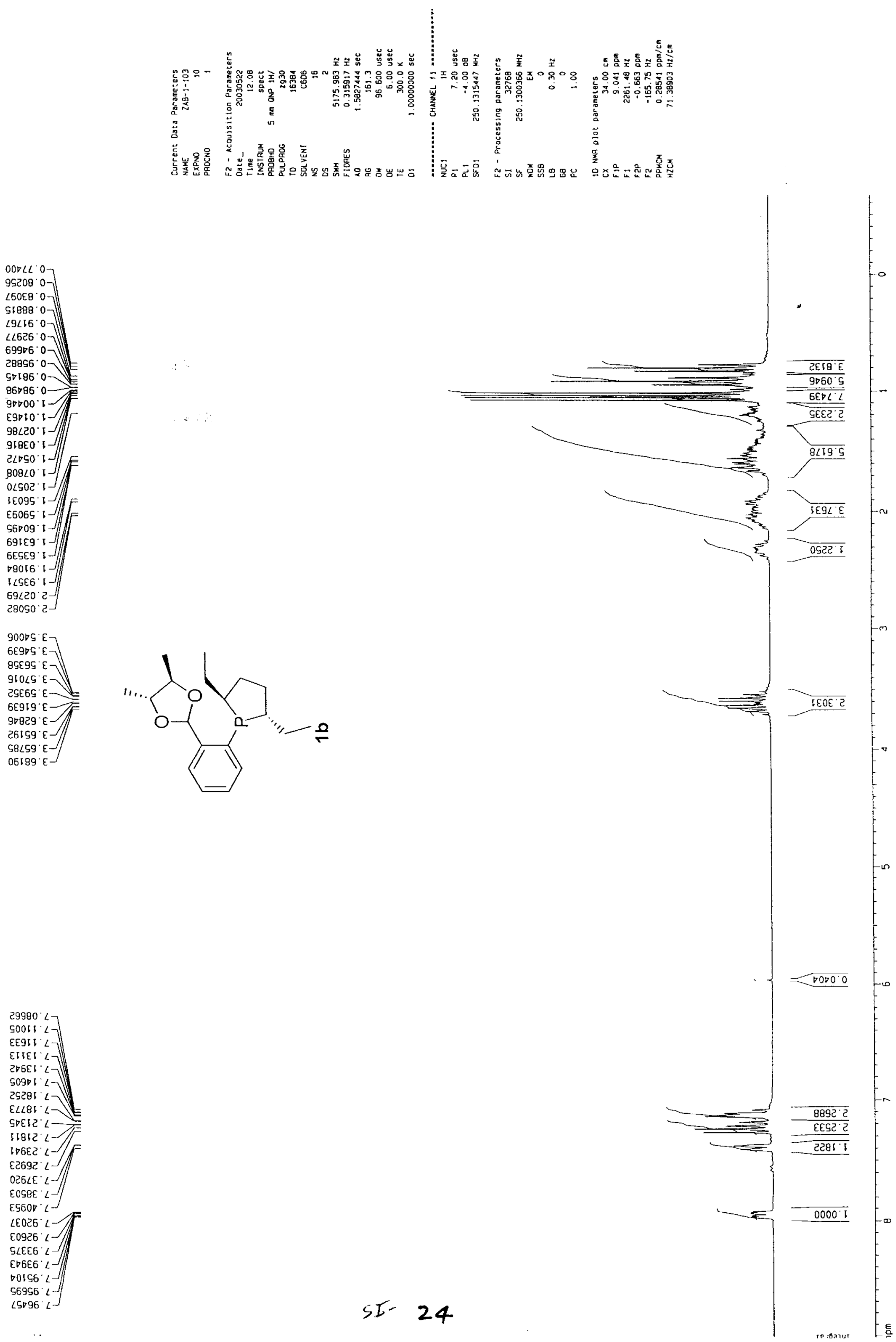

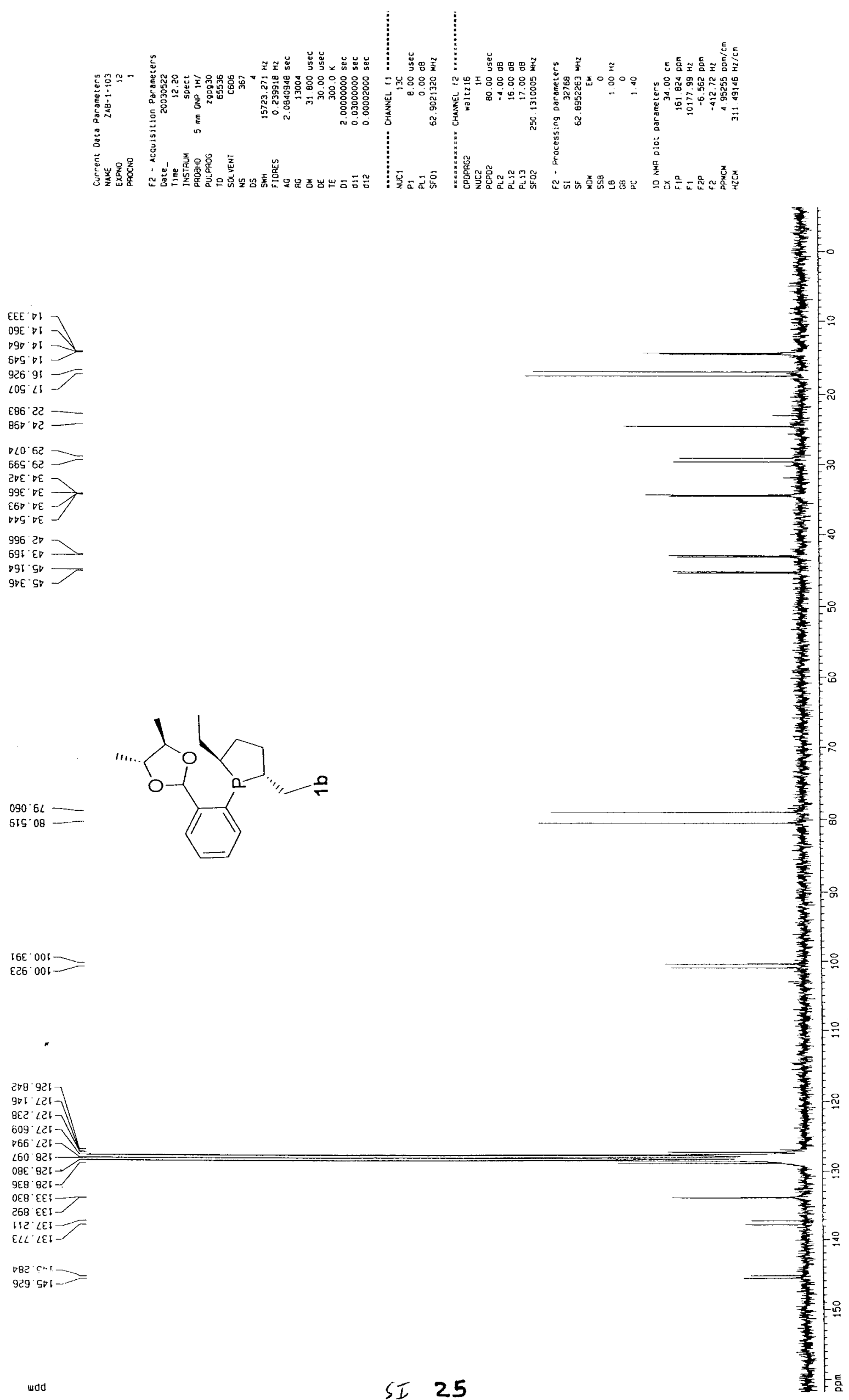

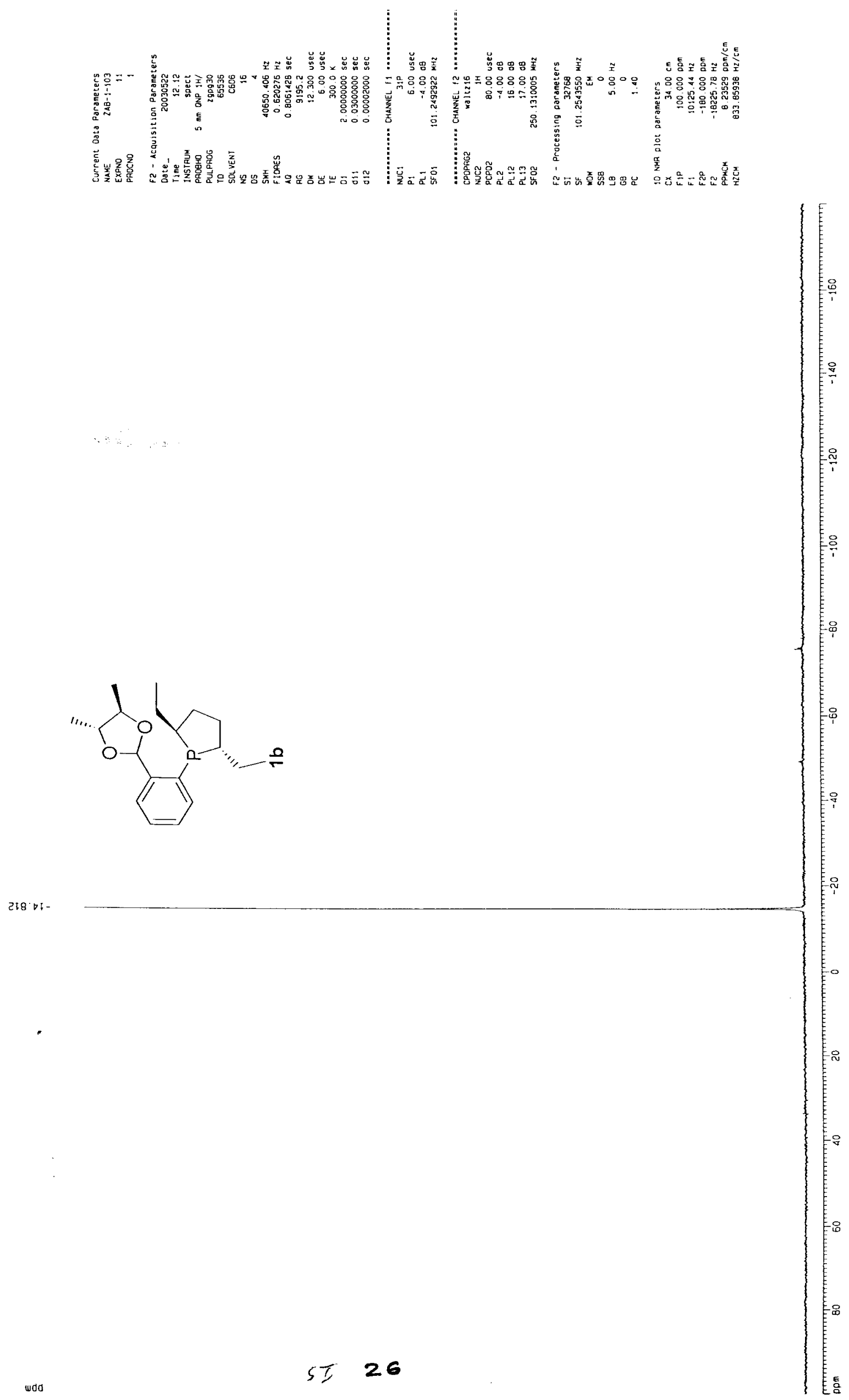

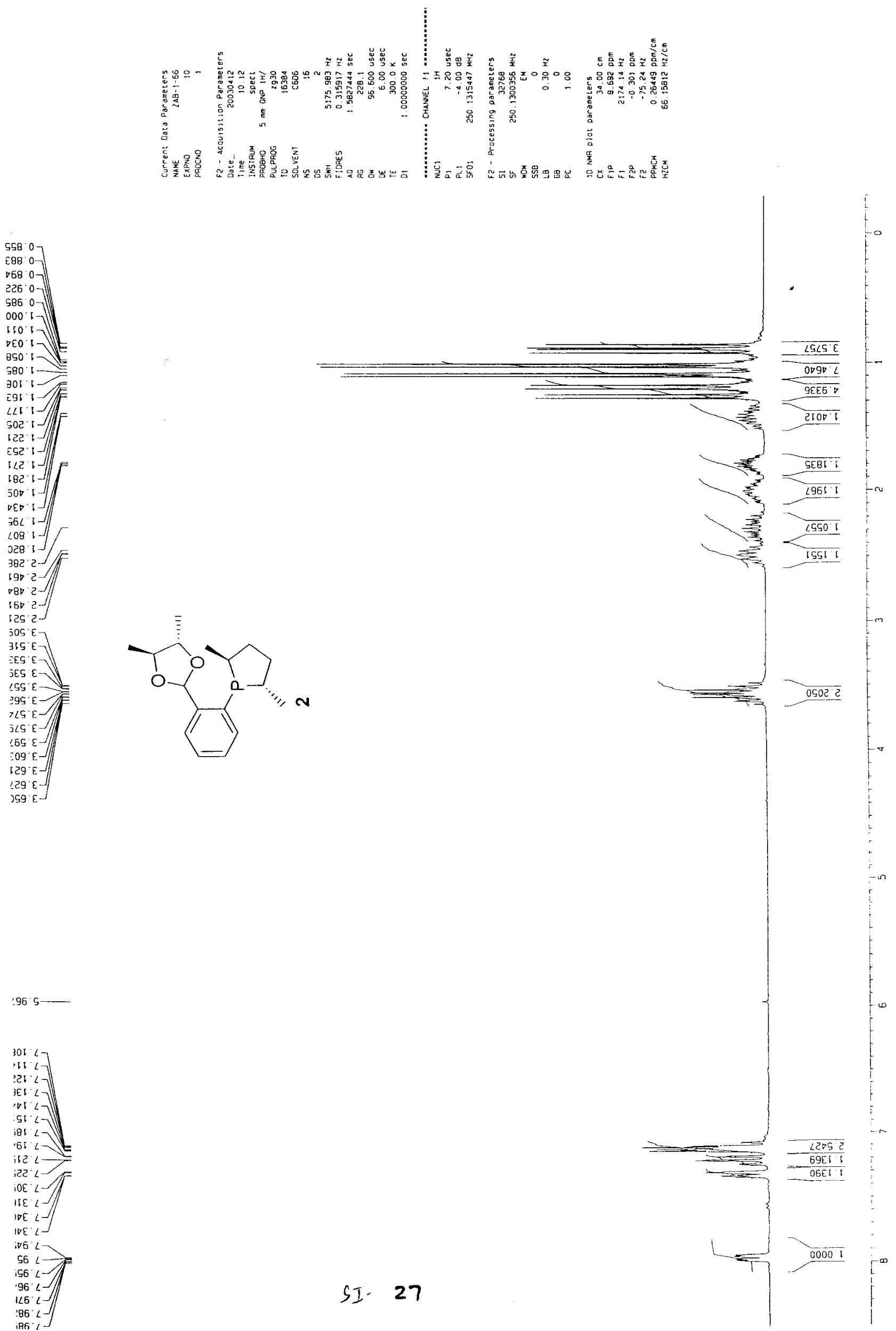

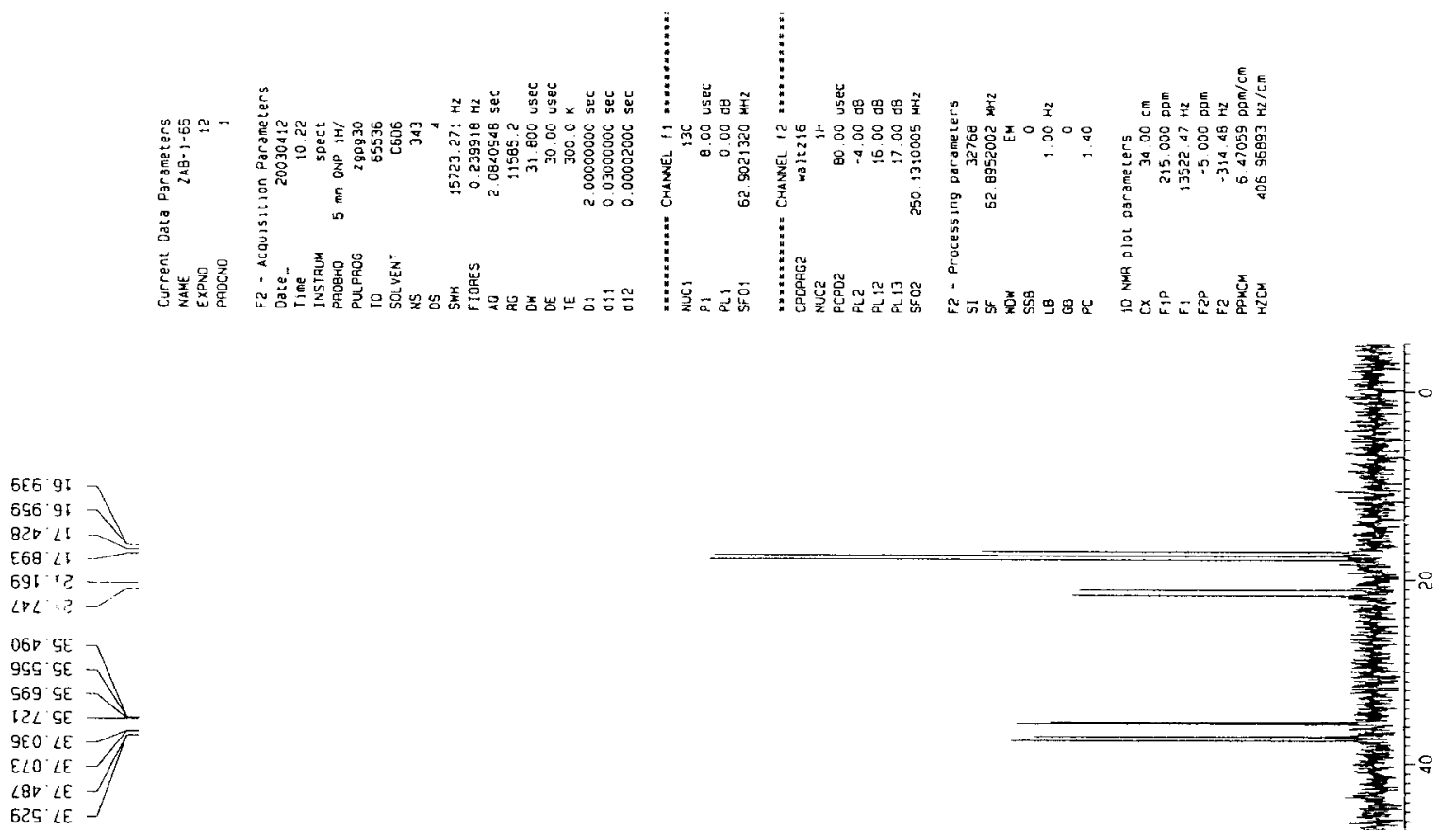

E80 62 20808
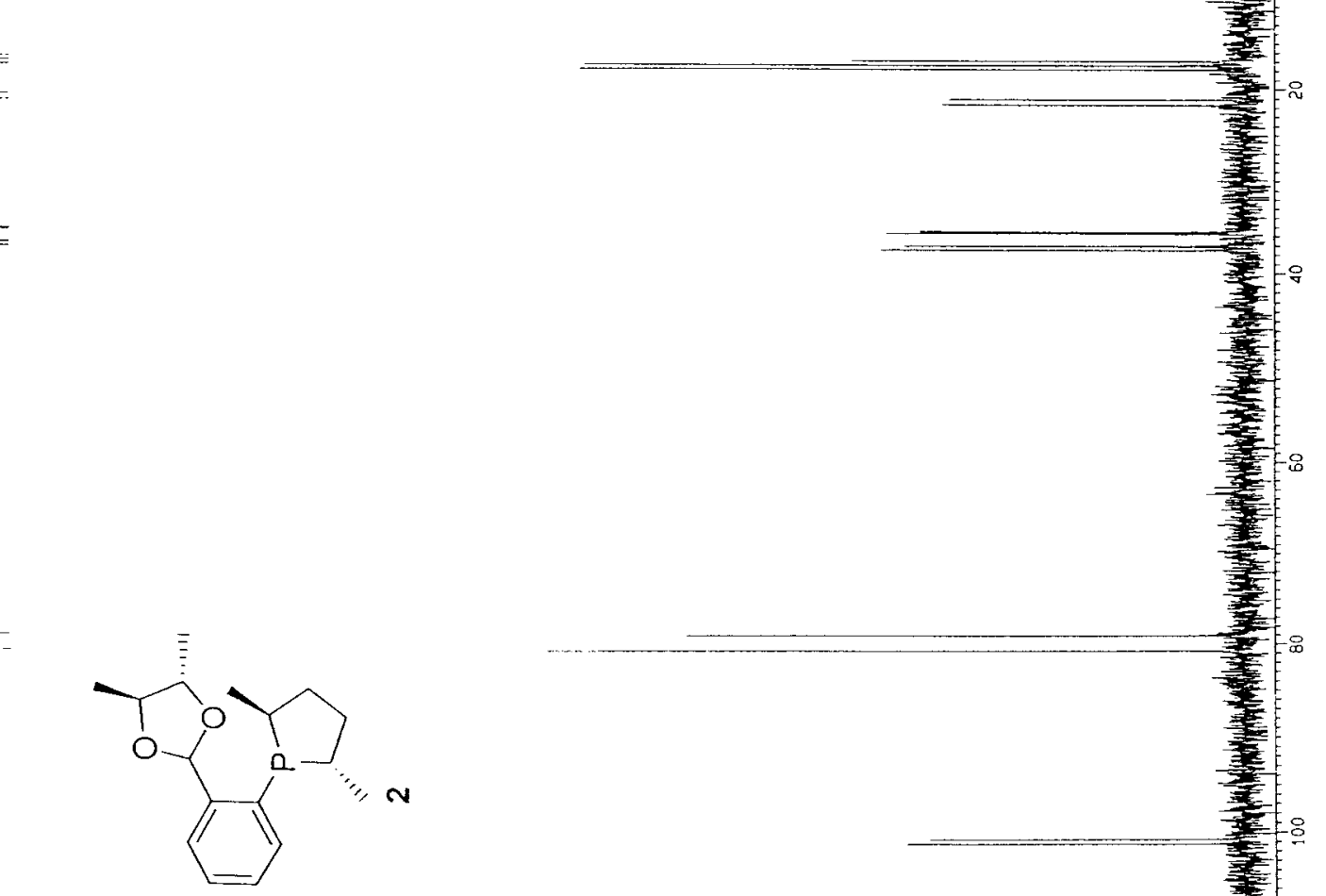

wdd 

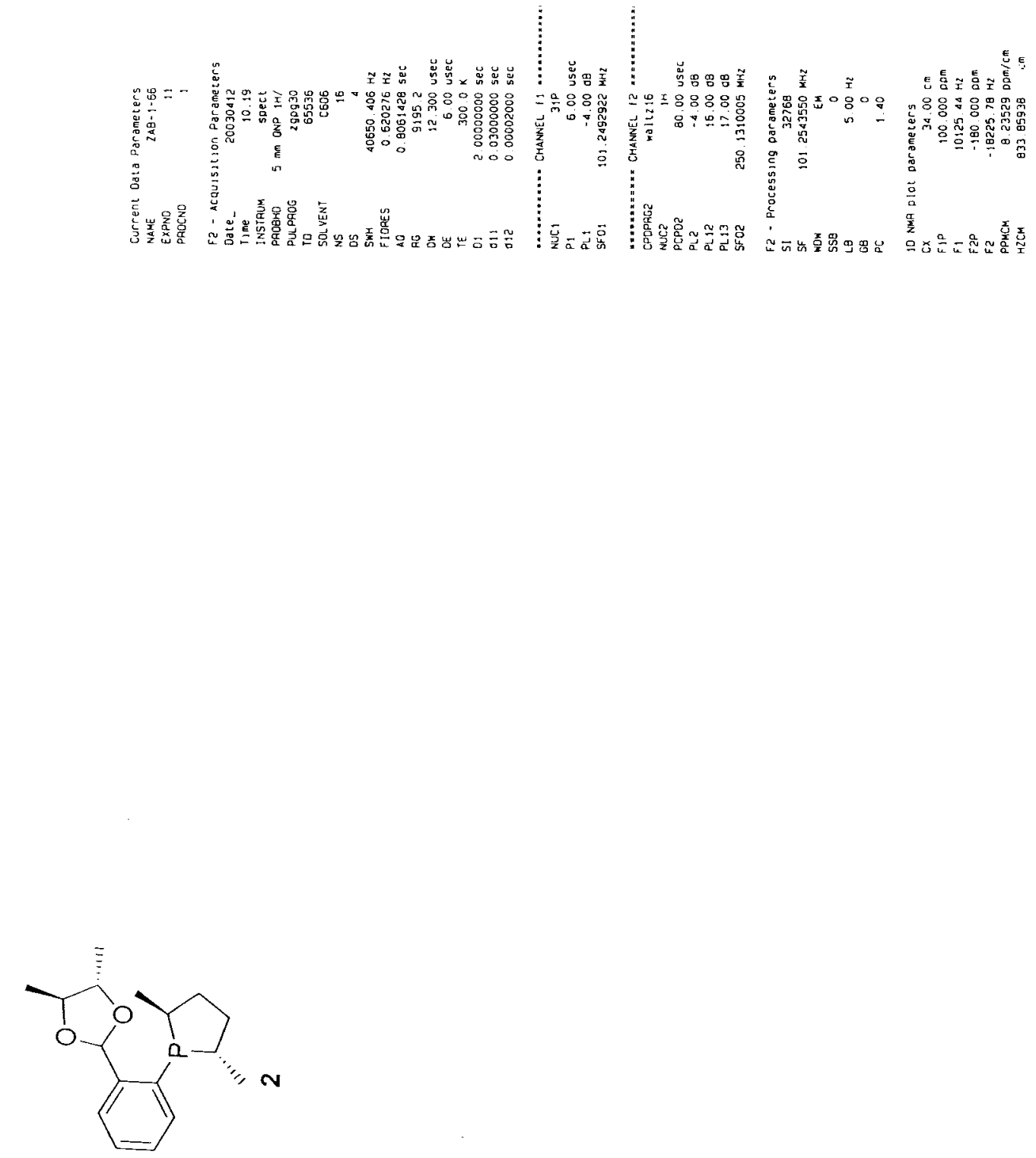


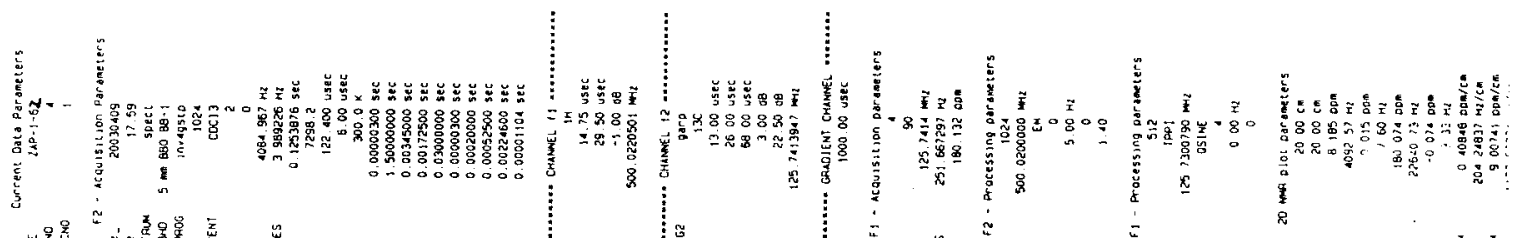

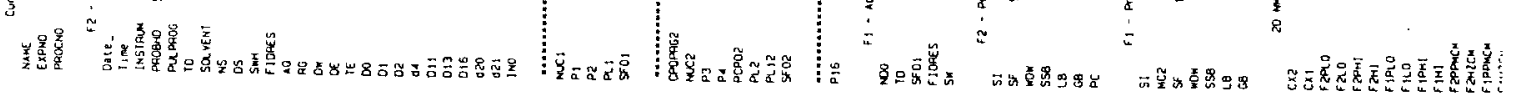
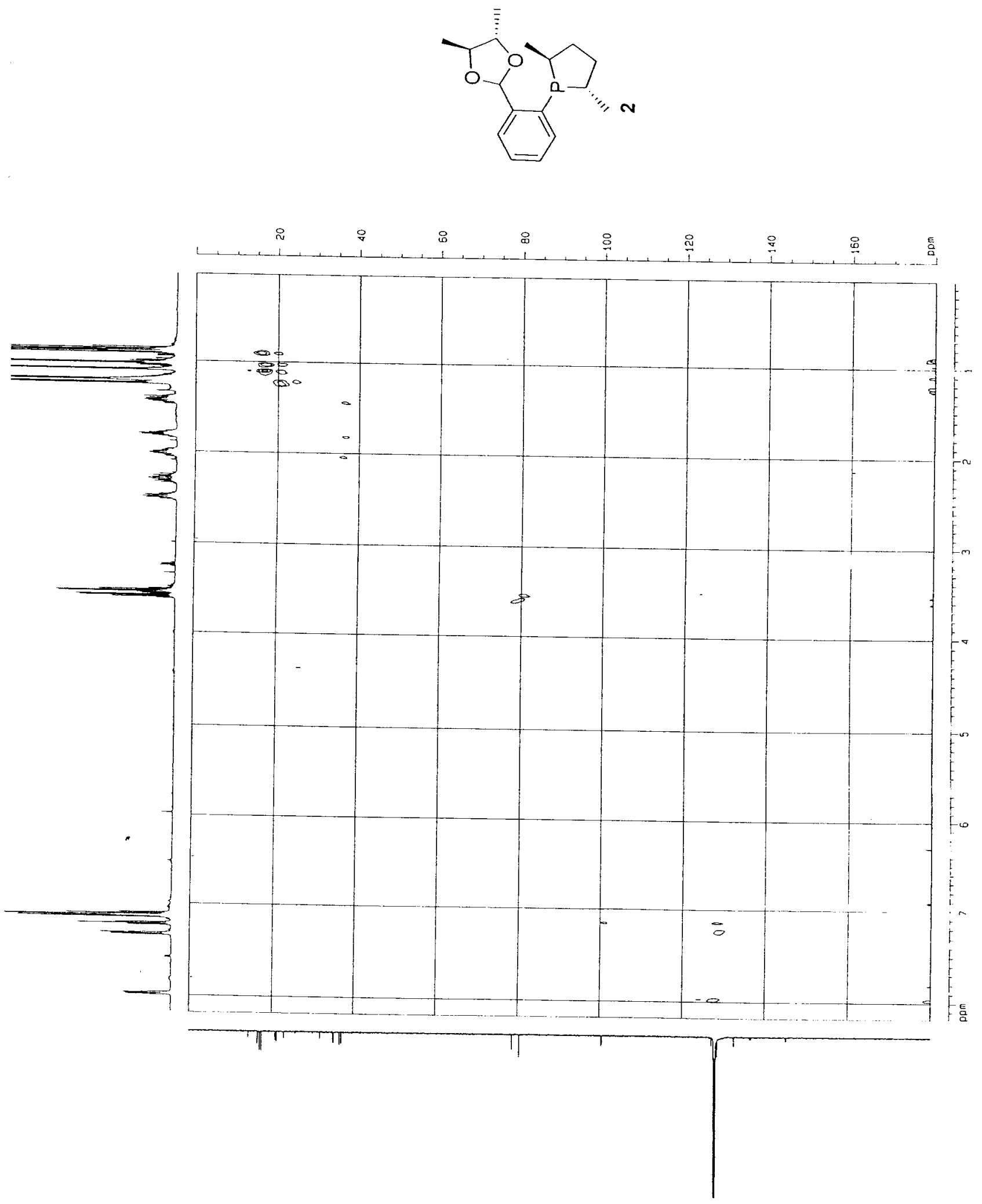

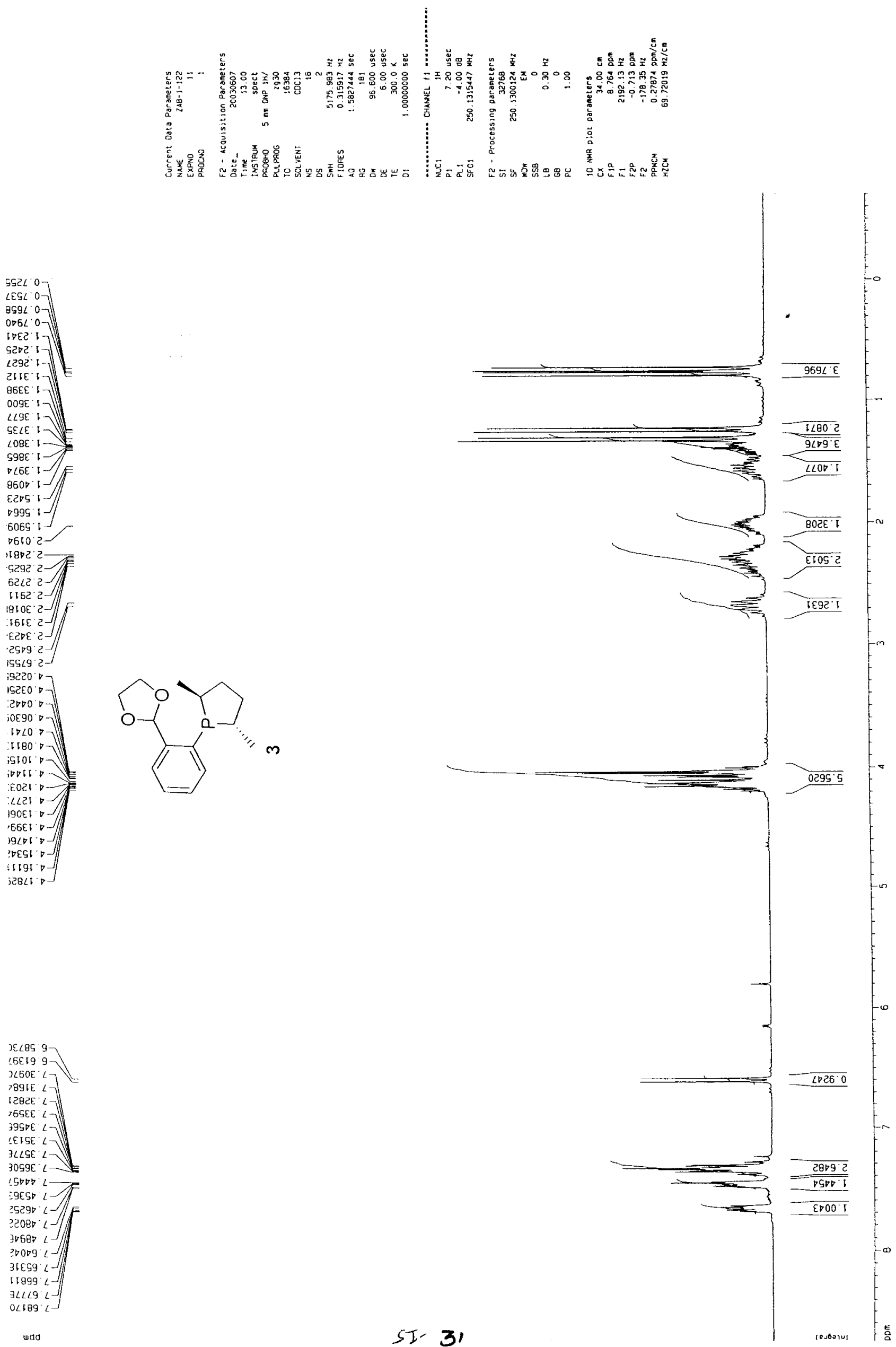

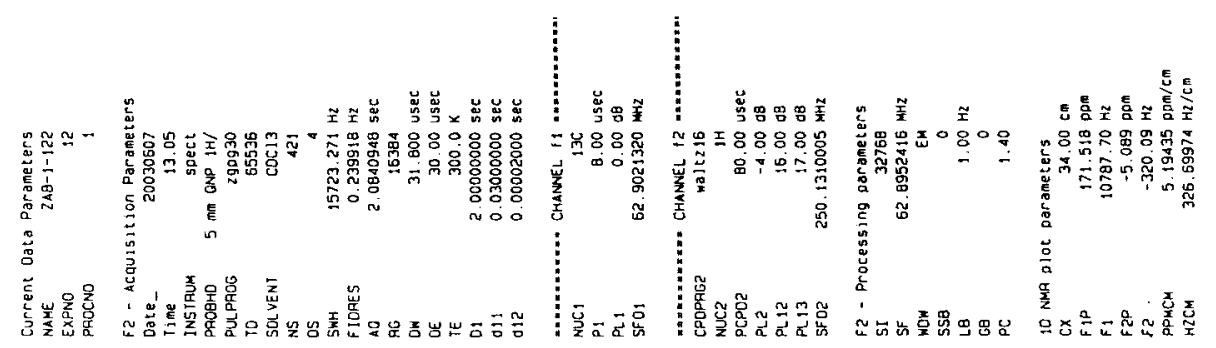

E96. $\mathrm{GI}$

$800.0 \%$

195.02

S21.12
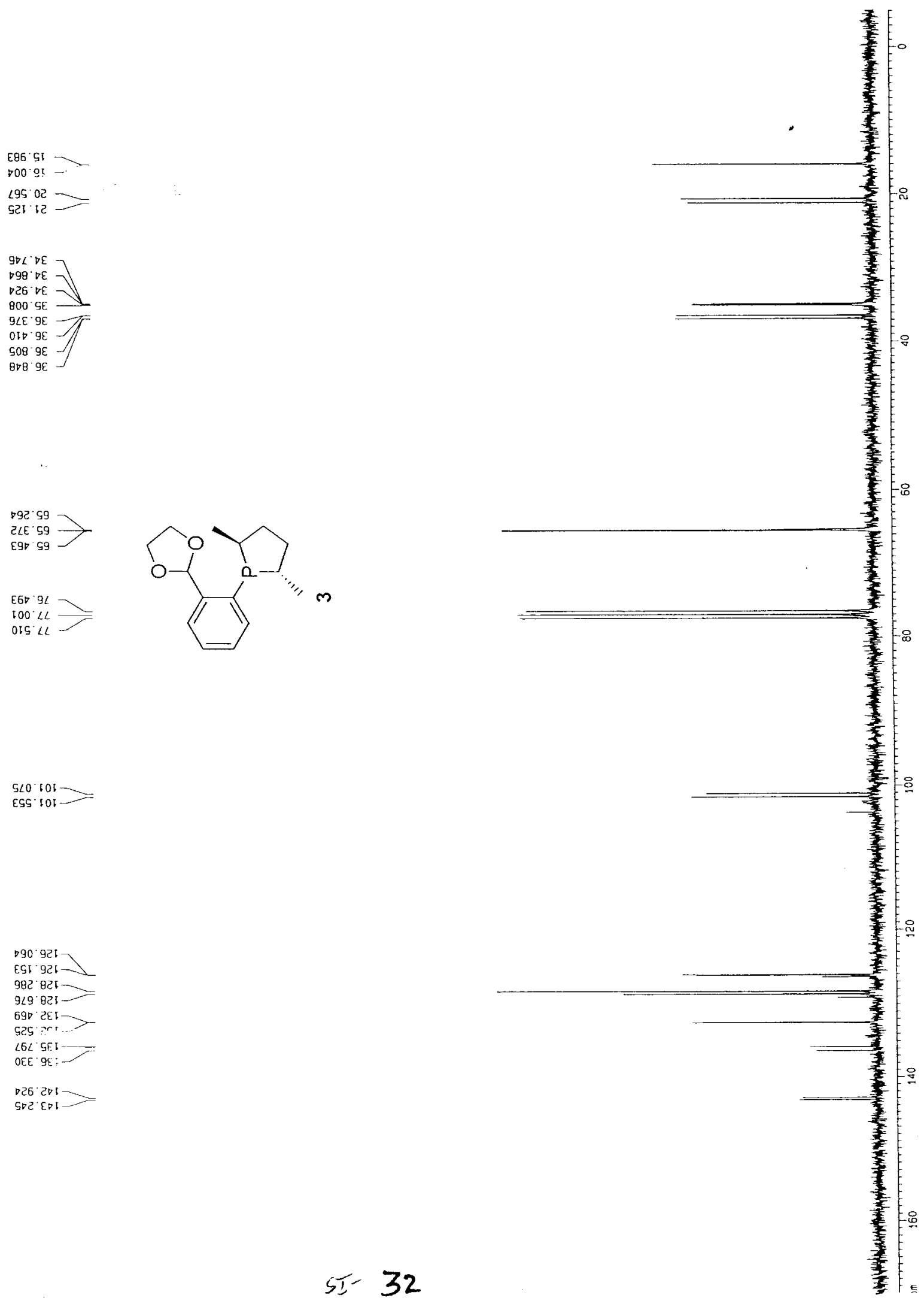

892.99
$2 L E \cdot 59$
E9D. 99

E6) $9 L$

$100 \angle L$

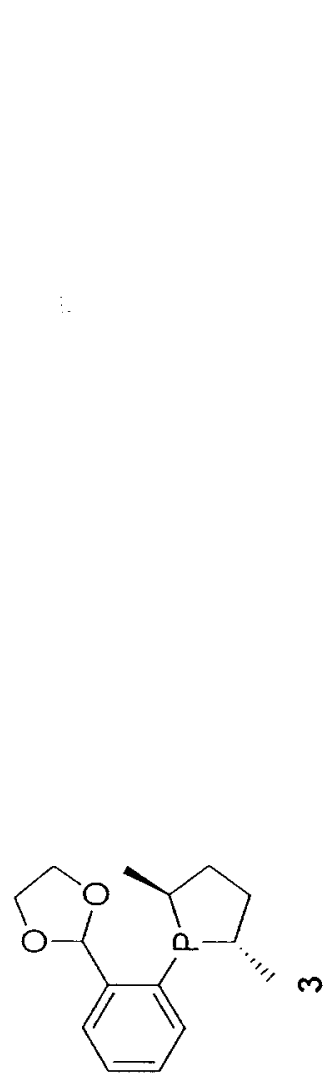

$5 \angle 0.601$

ESS.101

$790.92 t$

ESF $921-$

$982 \cdot 851-2$

$9 \angle 9.825$

69० $2 E$.

Ges ort ... $>$

OEE $9 E:-$

D56.501 

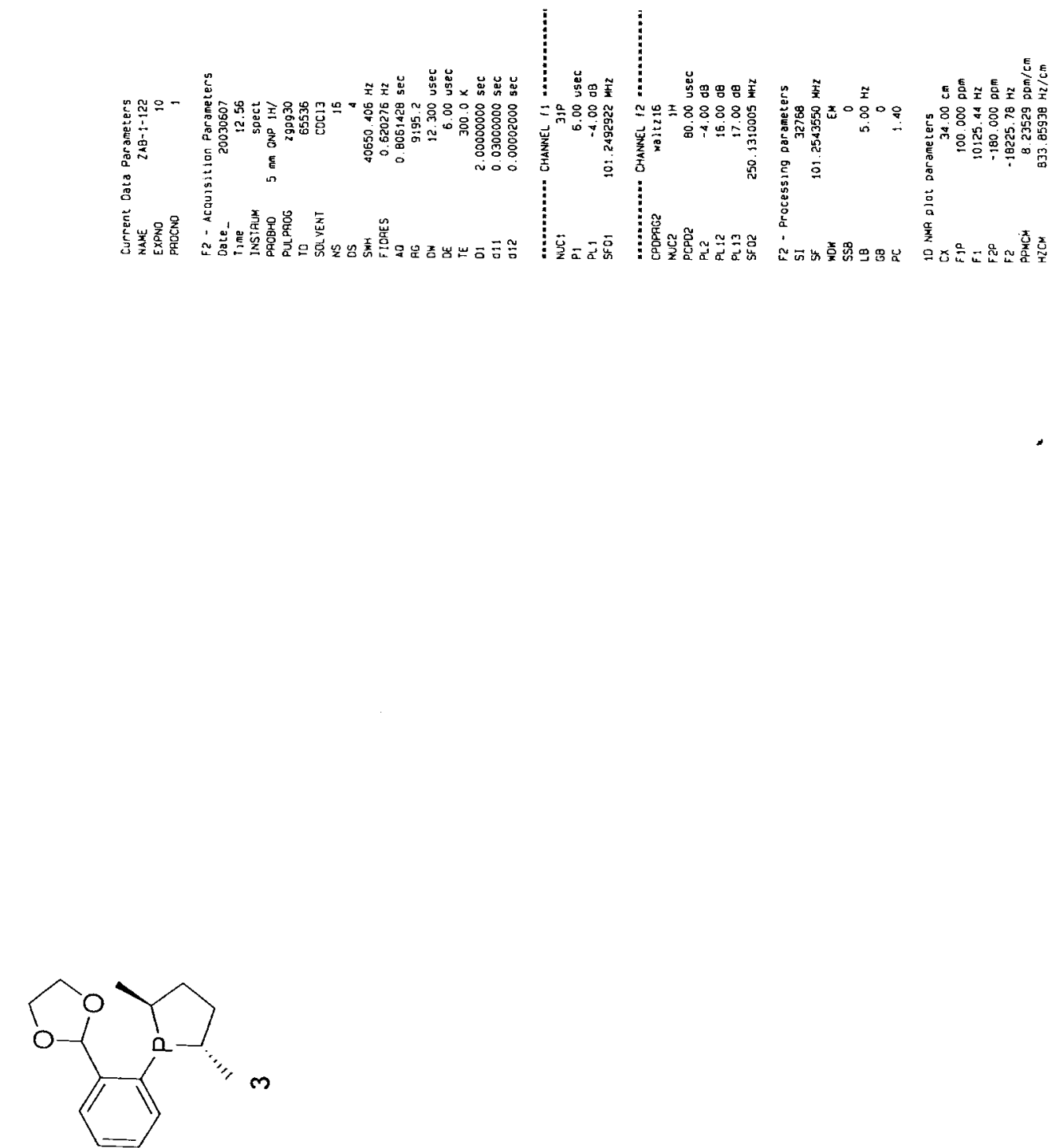

0969 r- 

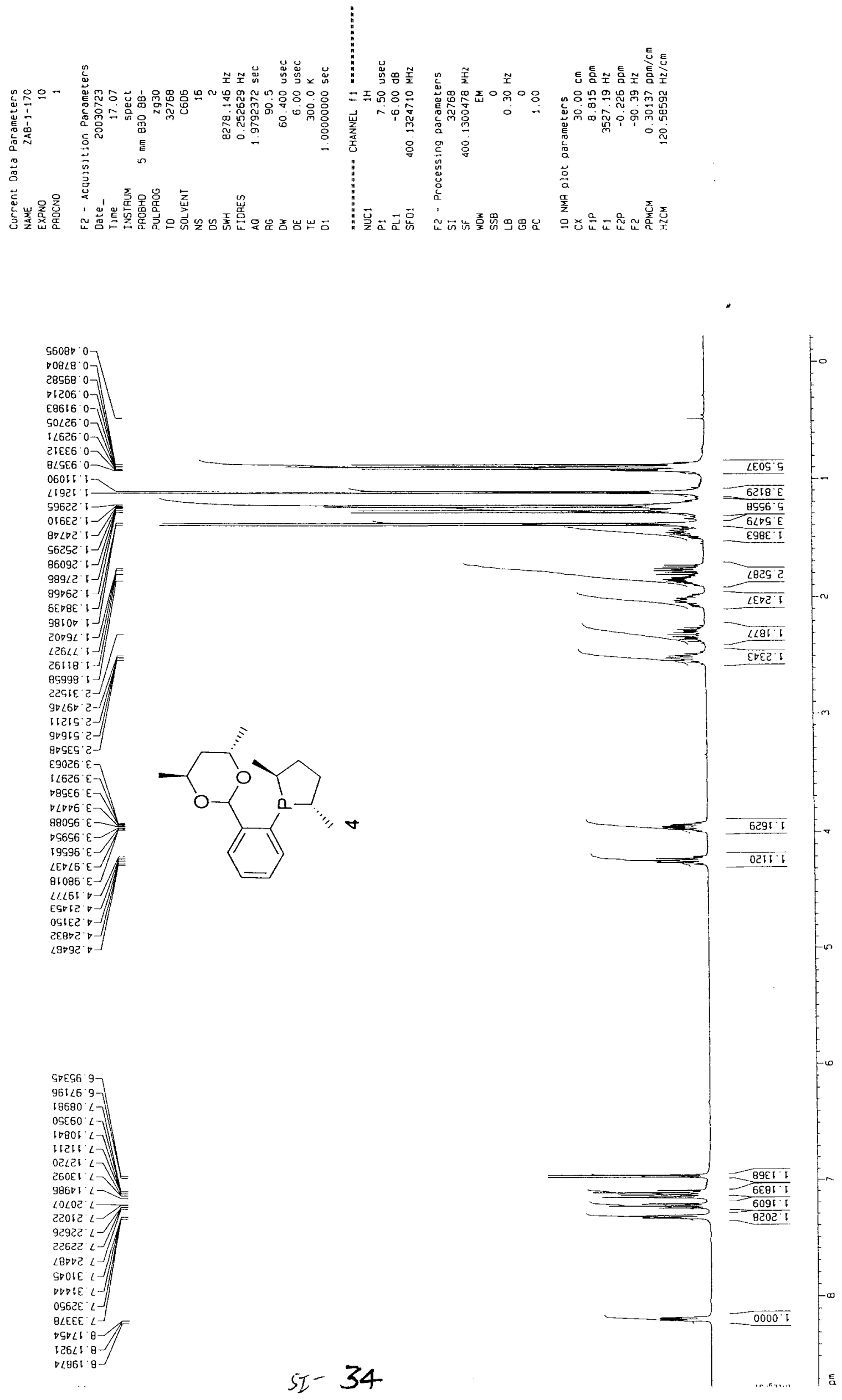


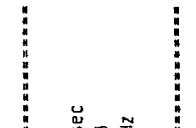

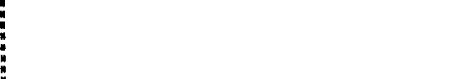
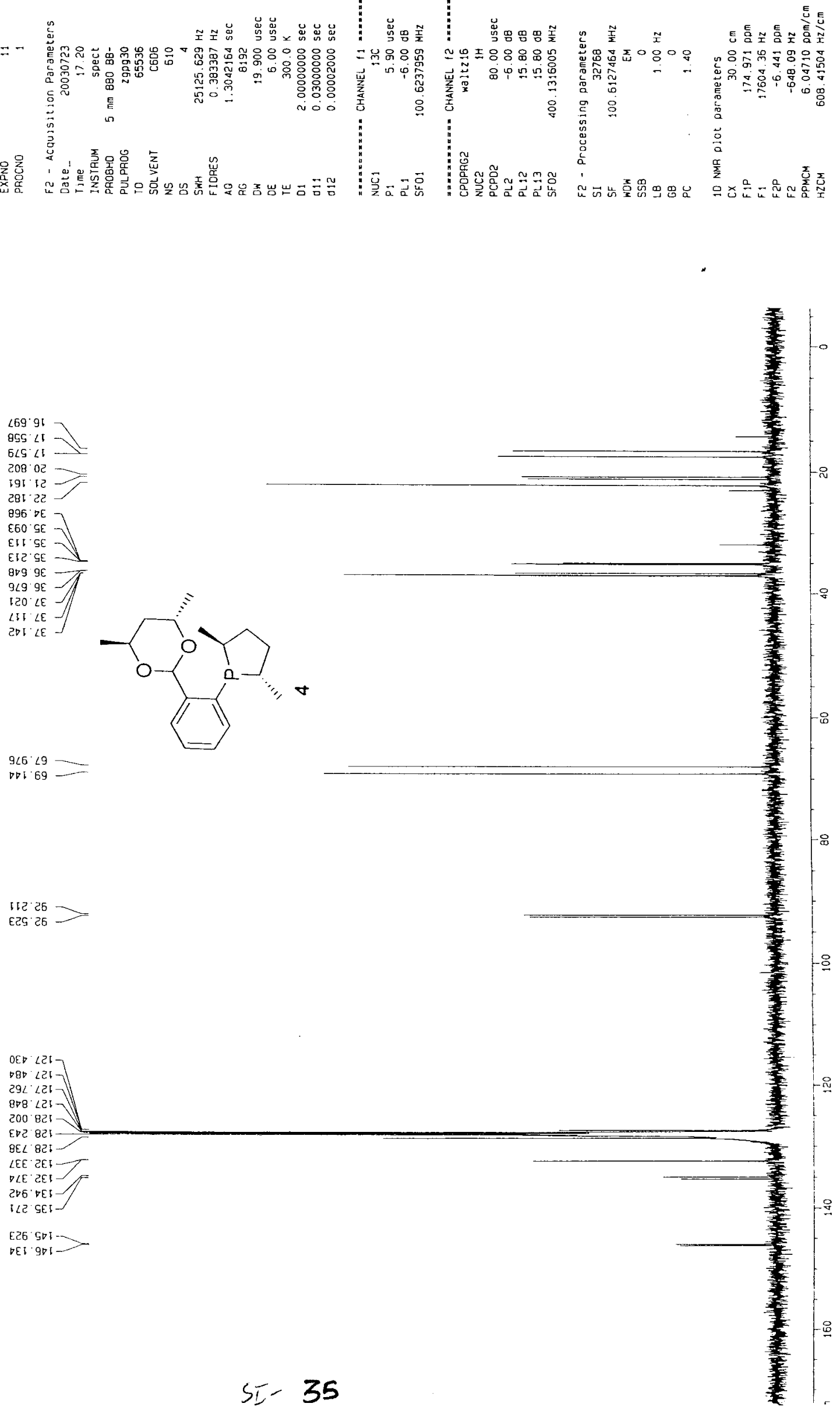


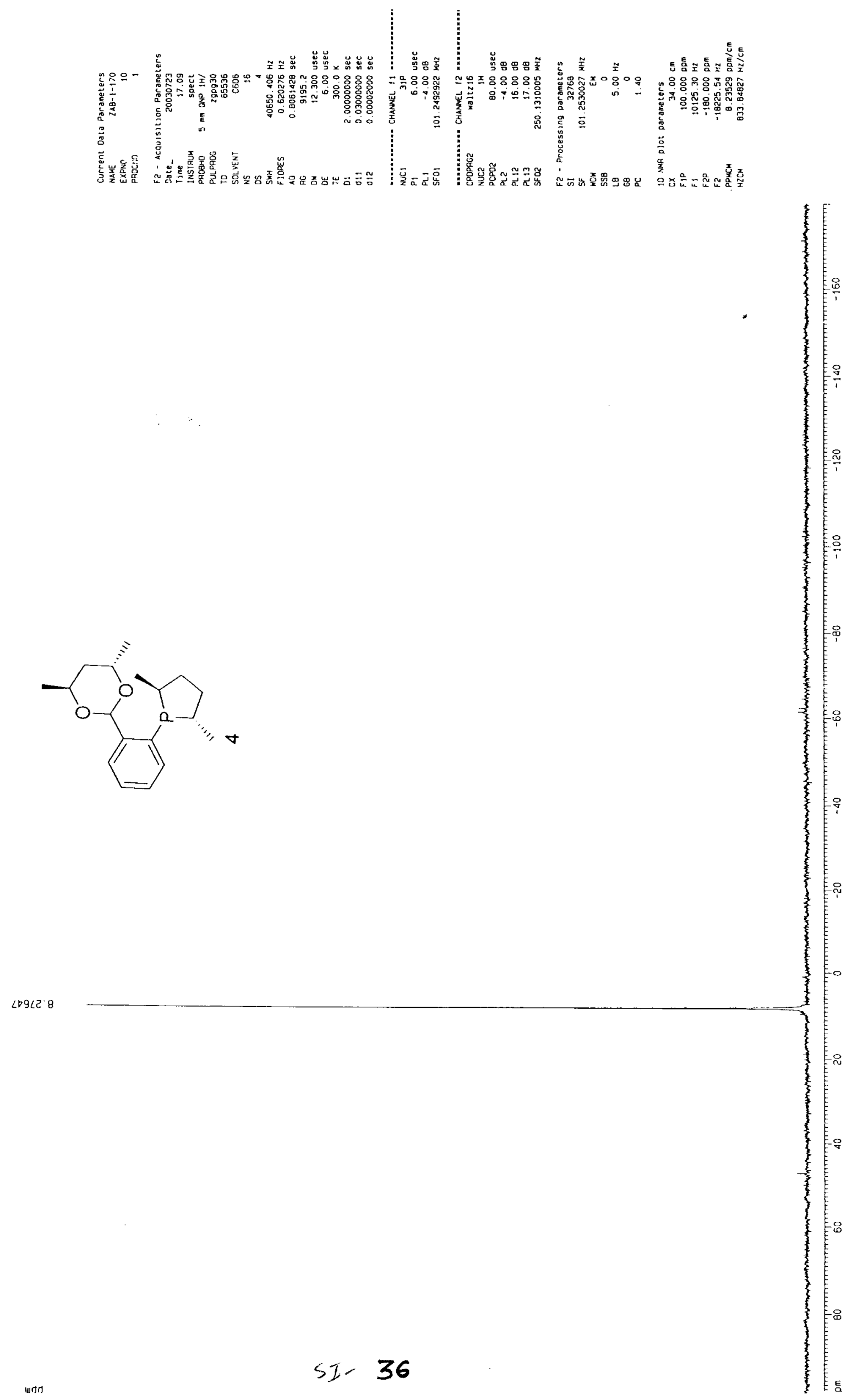



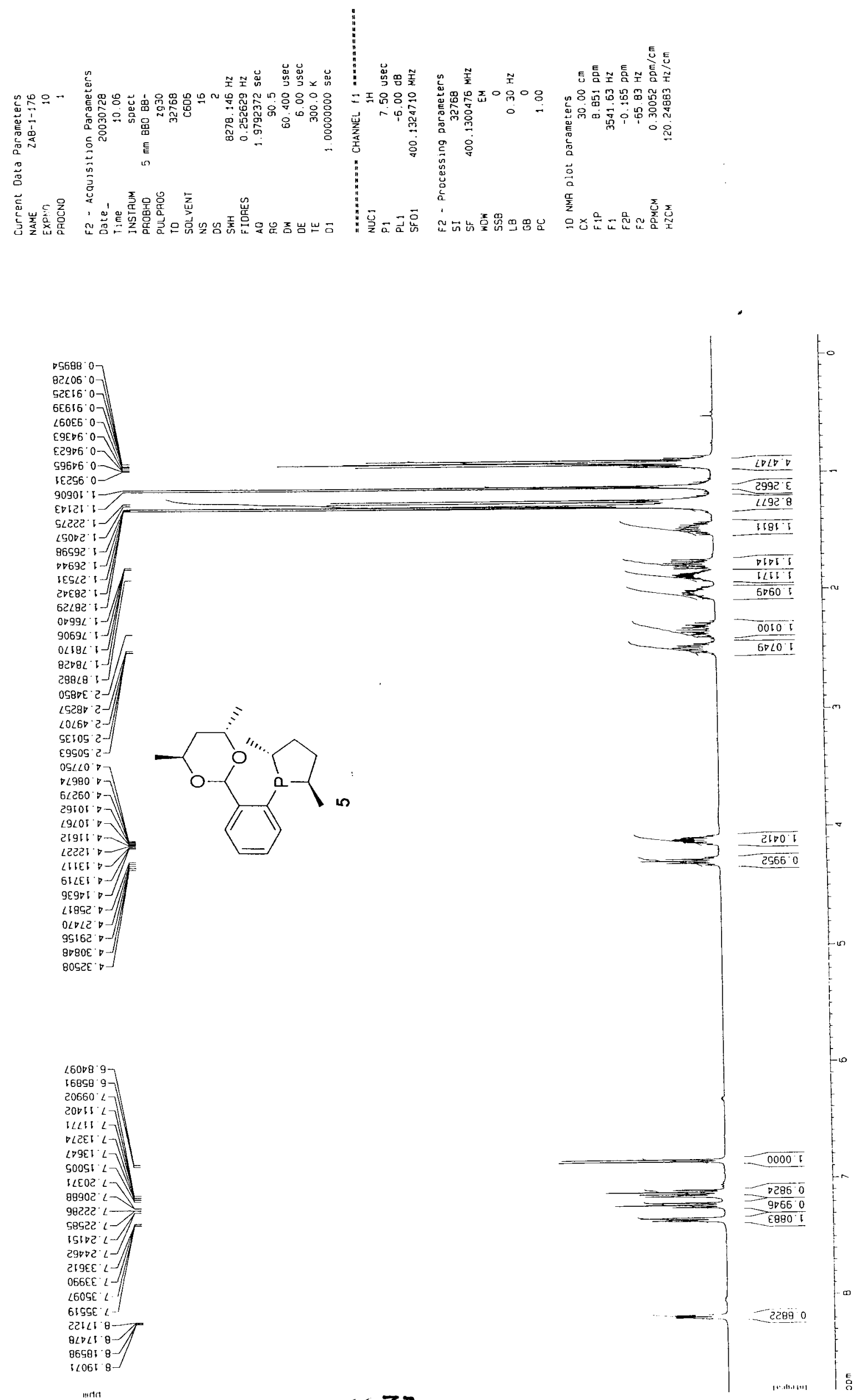

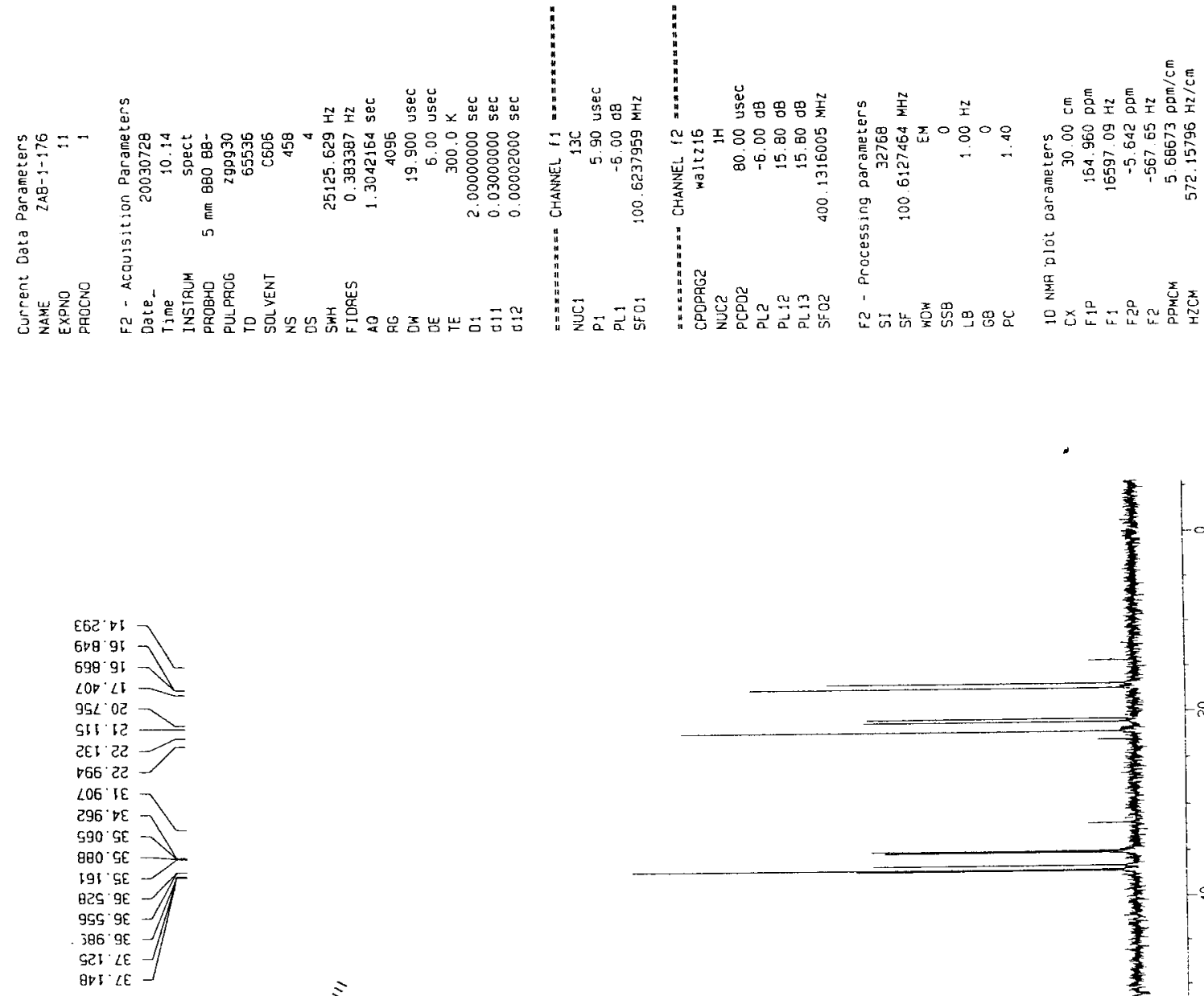

$00 \angle \angle 9-$
$69 \angle 89-$

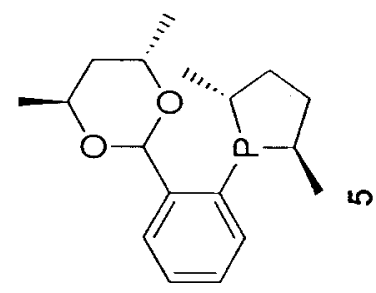

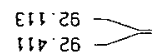

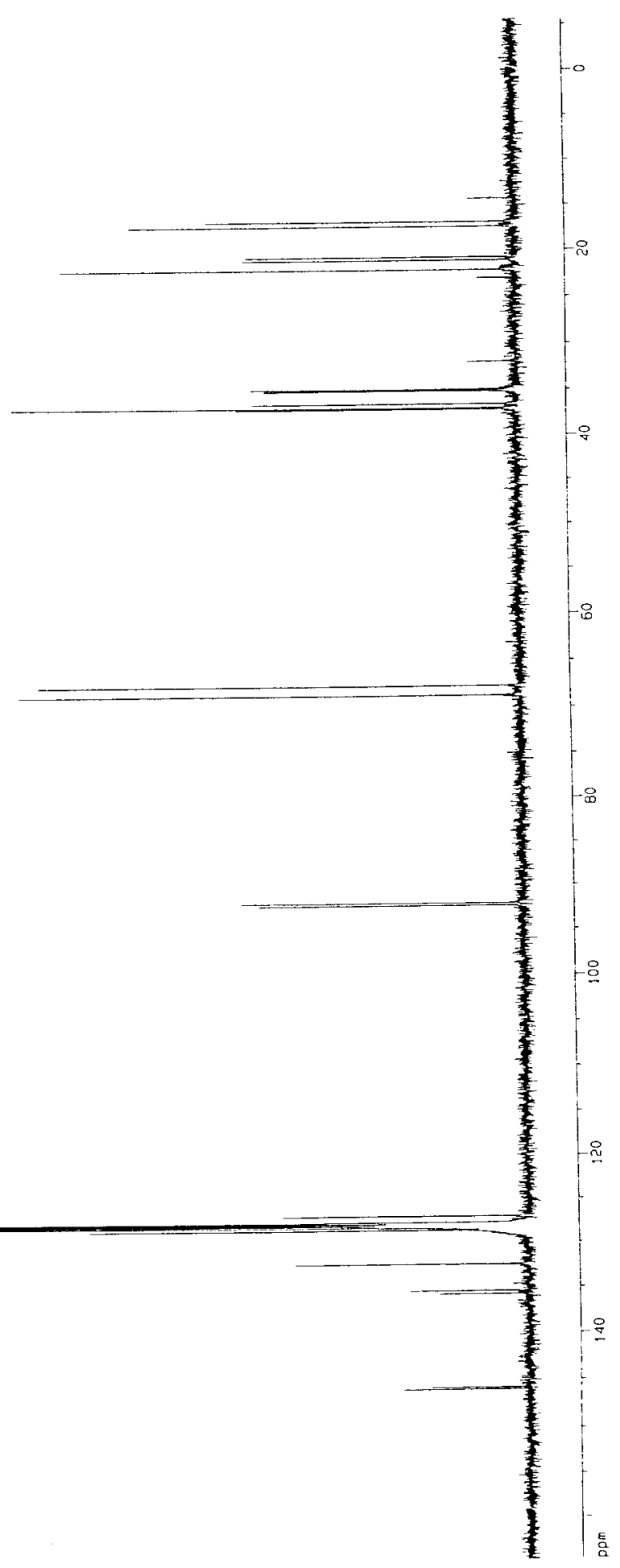



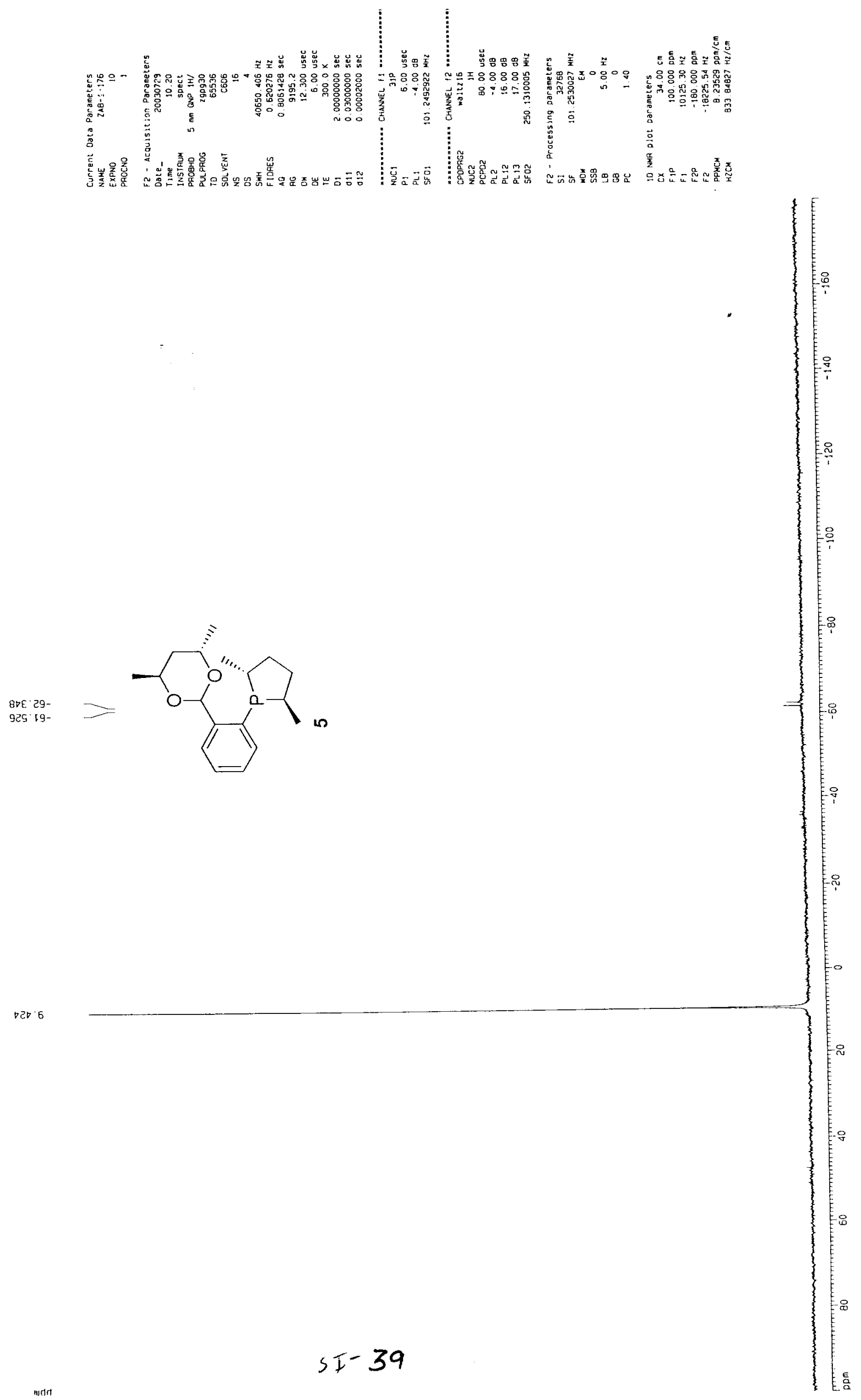

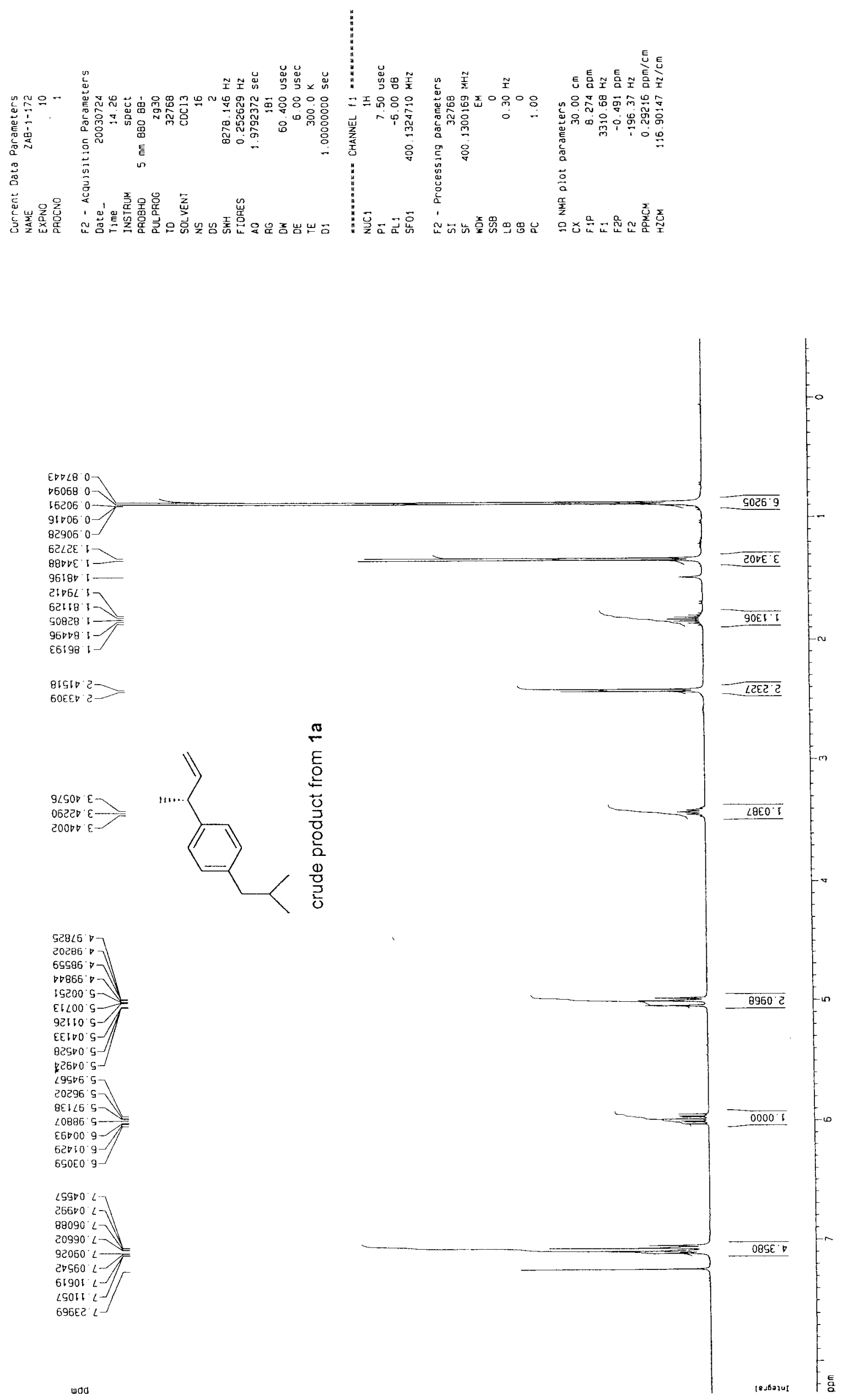


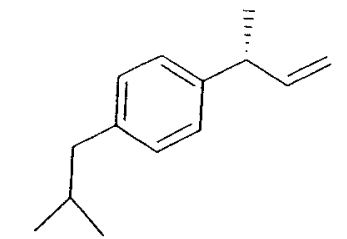

crude product from 1a

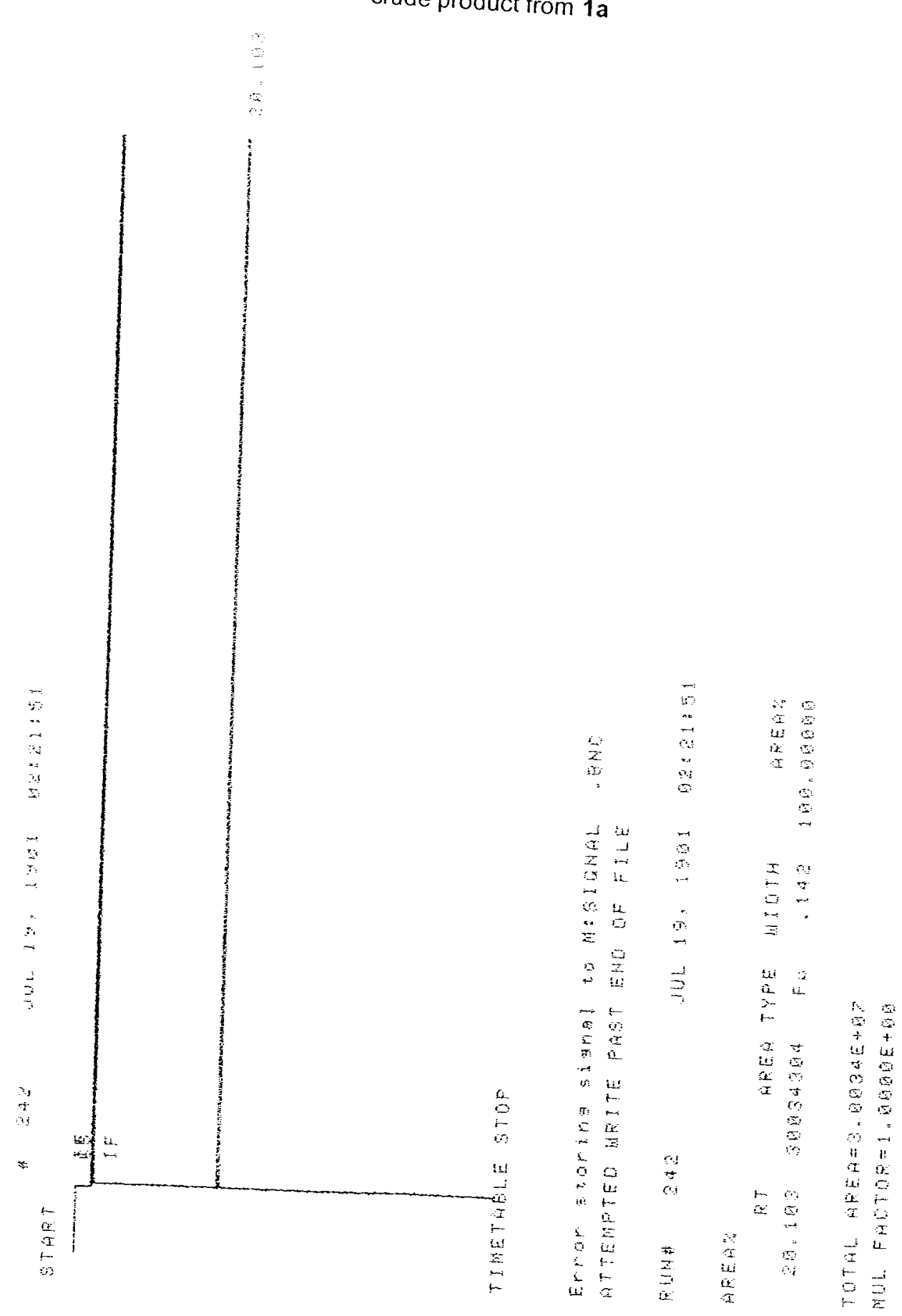

$1.0 \mathrm{~mL}$ helium $/ \mathrm{min}, 10 \mathrm{~min}$ at $100^{\circ} \mathrm{C}, 4^{\circ} \mathrm{C} / \mathrm{min}, 10 \mathrm{~min}$ at $200^{\circ} \mathrm{C}$ 

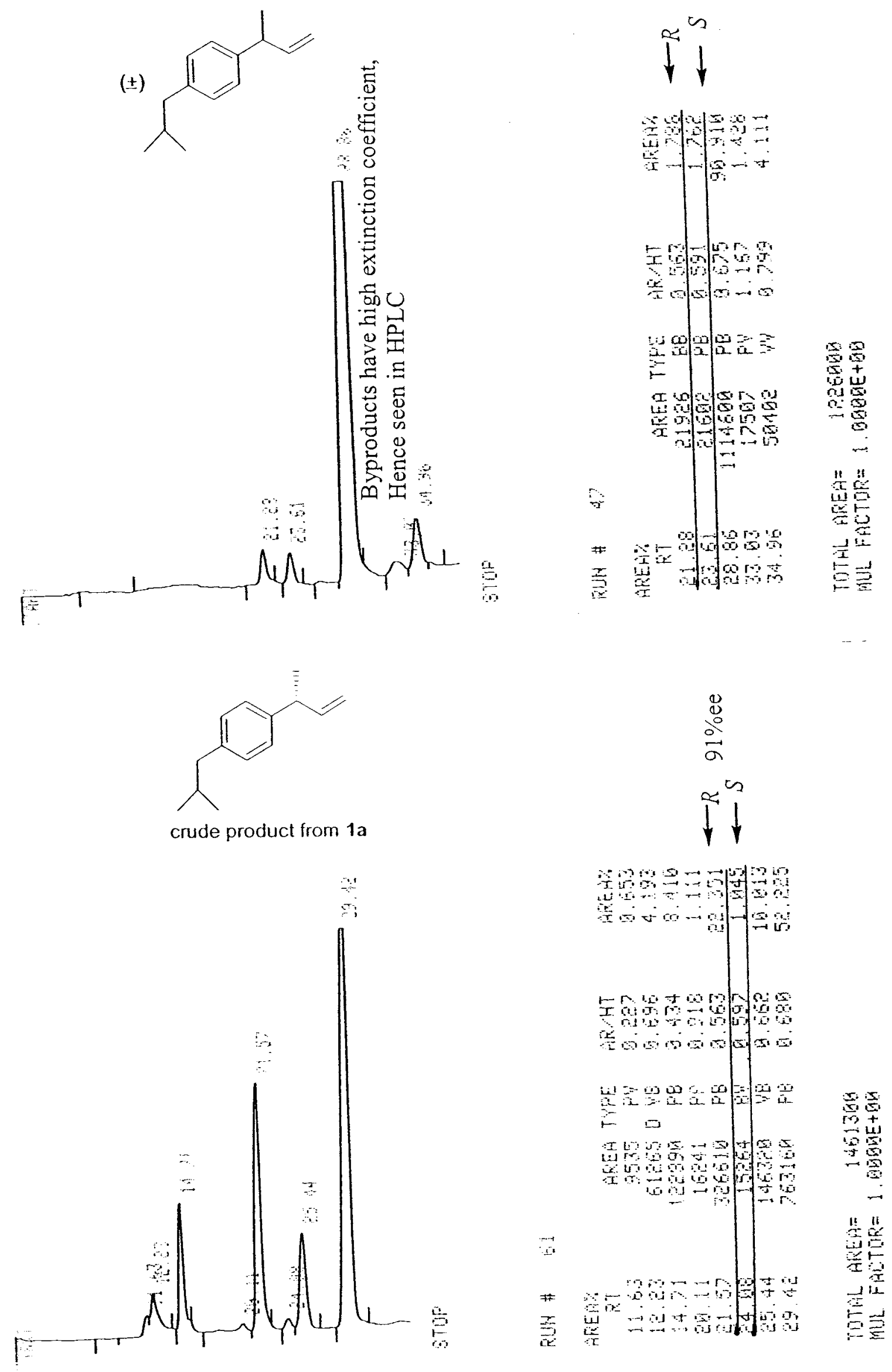

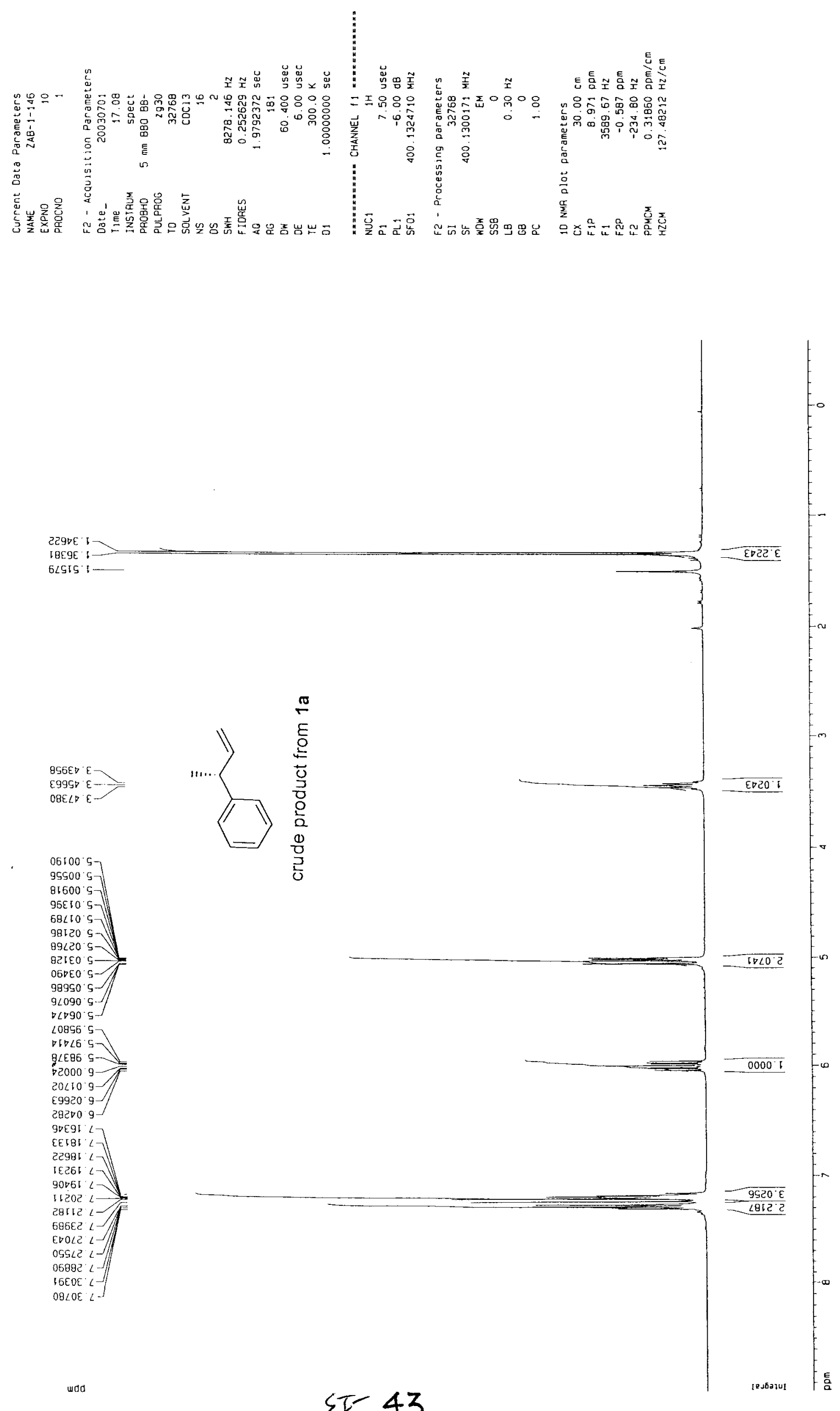
<smiles>C=C[C@H](C)c1ccccc1</smiles>

crude product from ia

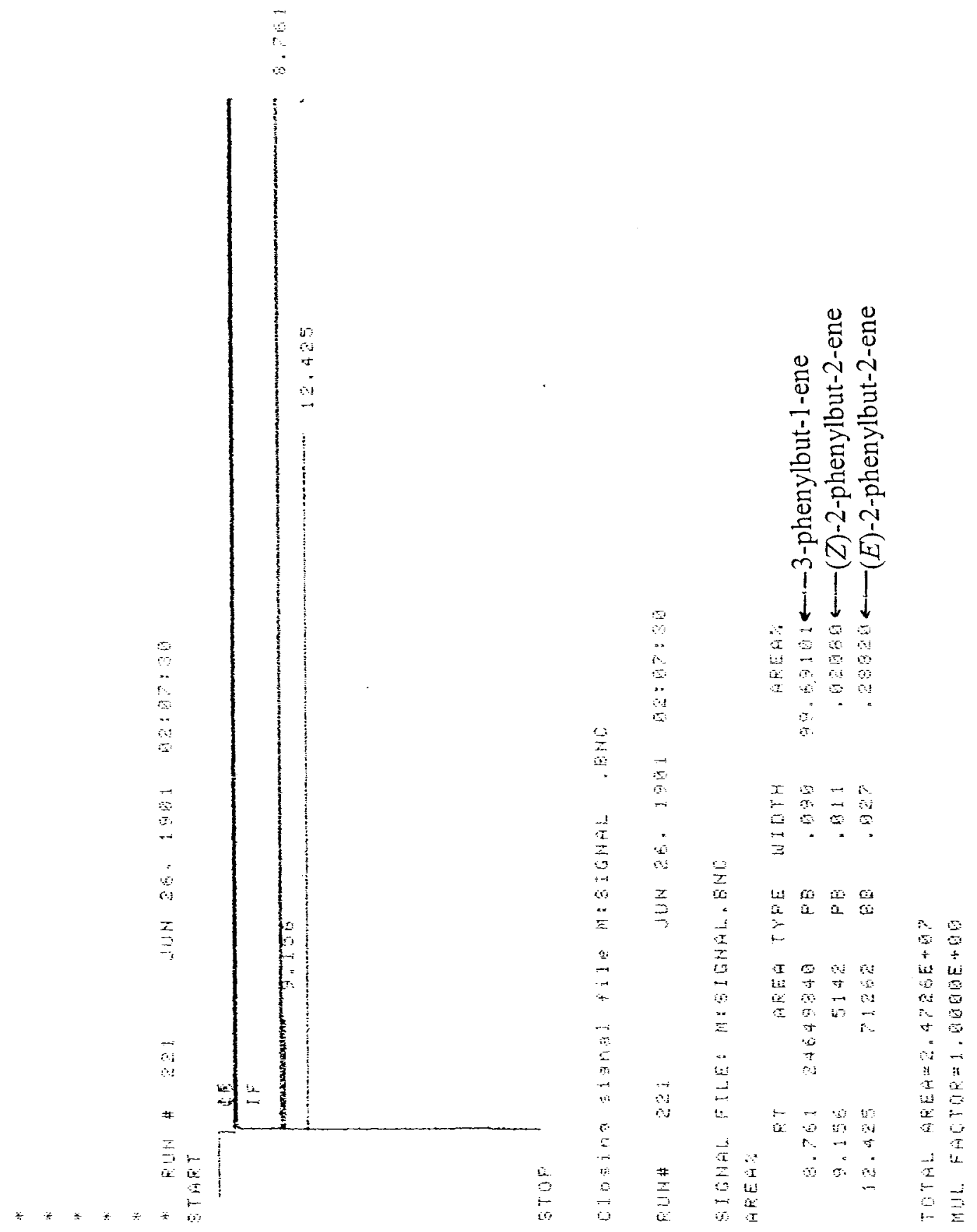

$1.0 \mathrm{~mL}$ helium $/ \mathrm{min}, 5 \mathrm{~min}$ at $80^{\circ} \mathrm{C}, 4^{\circ} \mathrm{C} / \mathrm{min}, 15 \mathrm{~min}$ at $180^{\circ} \mathrm{C}$

SI 44 

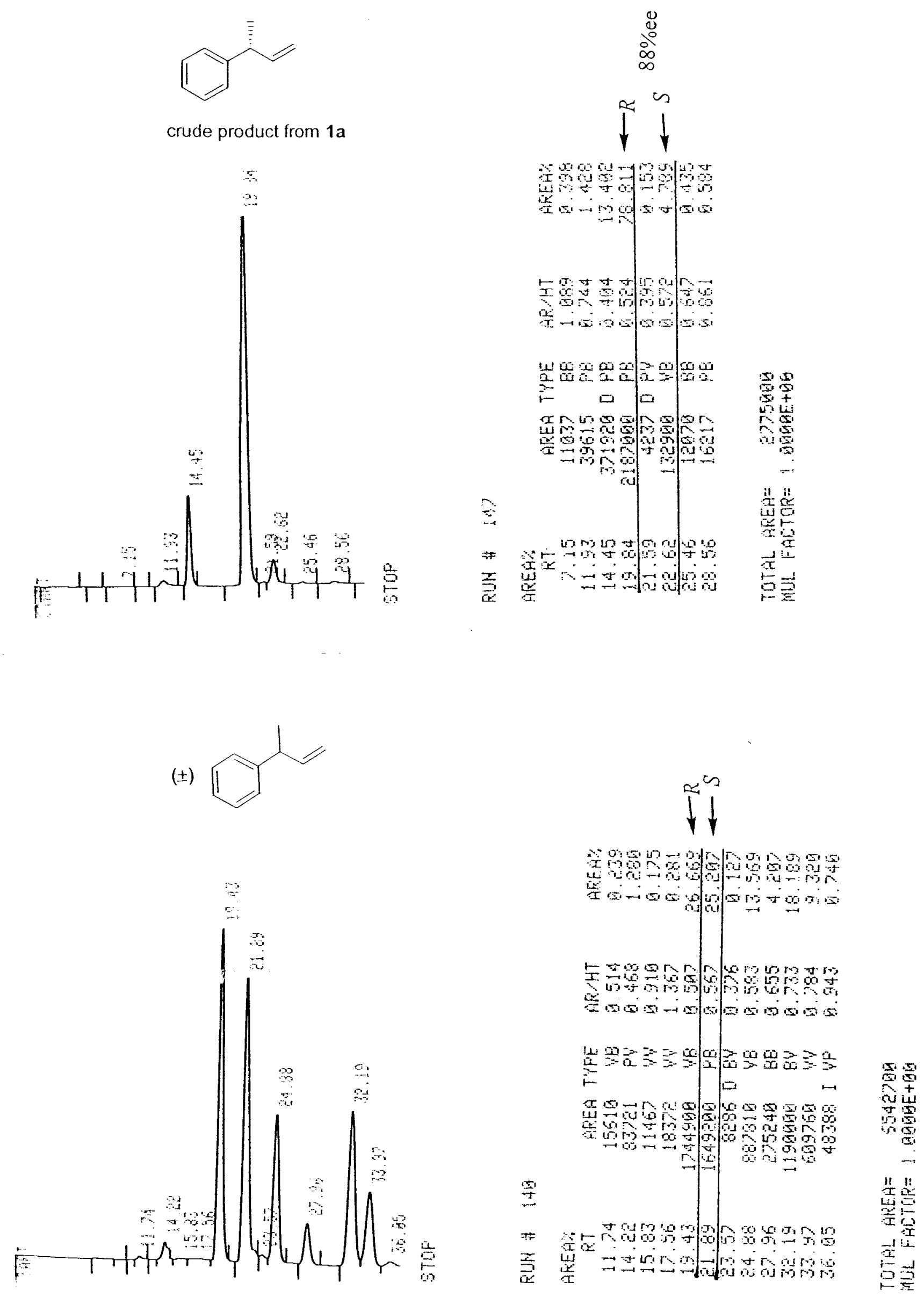

Daicel Chiralcel OJ Column, $100 \%$ hexane, $0.30 \mathrm{~mL} / \mathrm{min}$ 


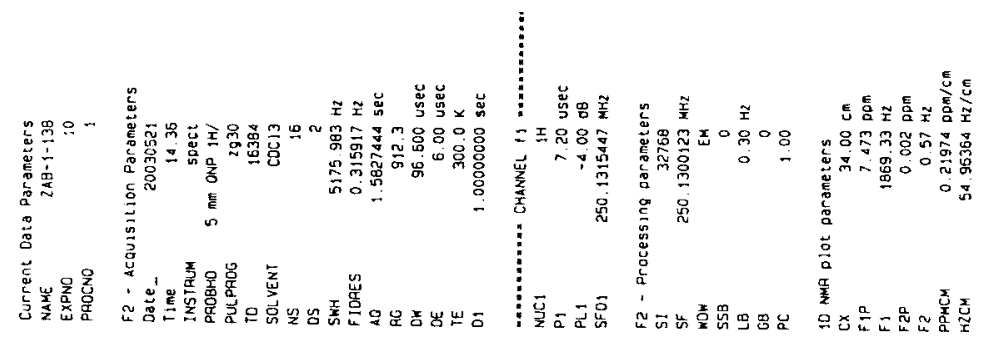

SIOLE' I-

DELEE I

ZGODE I

$60 \angle 051$

$96566^{\circ} 1-$

E0966 1

$921002-$

SO9OE: $2-$

5०GEE: 2

$0916 E^{-} \varepsilon-$

จ

$50900 \mathrm{E}-$

6L896

$605 \angle 6^{\circ} \circ 7$

$\left.6886^{\circ} \circ\right]$

$50566 \circ$

68600.5

$909105-$

L $\angle 20 \mathrm{~S}-$

$00 \angle 50 \mathrm{~s}-$

[ $\angle E 90^{\circ} \mathrm{s}$

$60016 \mathrm{~s}$

EE0v6 $\mathrm{S}$

19956.9

$92286 \div$

$98800^{\circ} 9-/$

02820.9

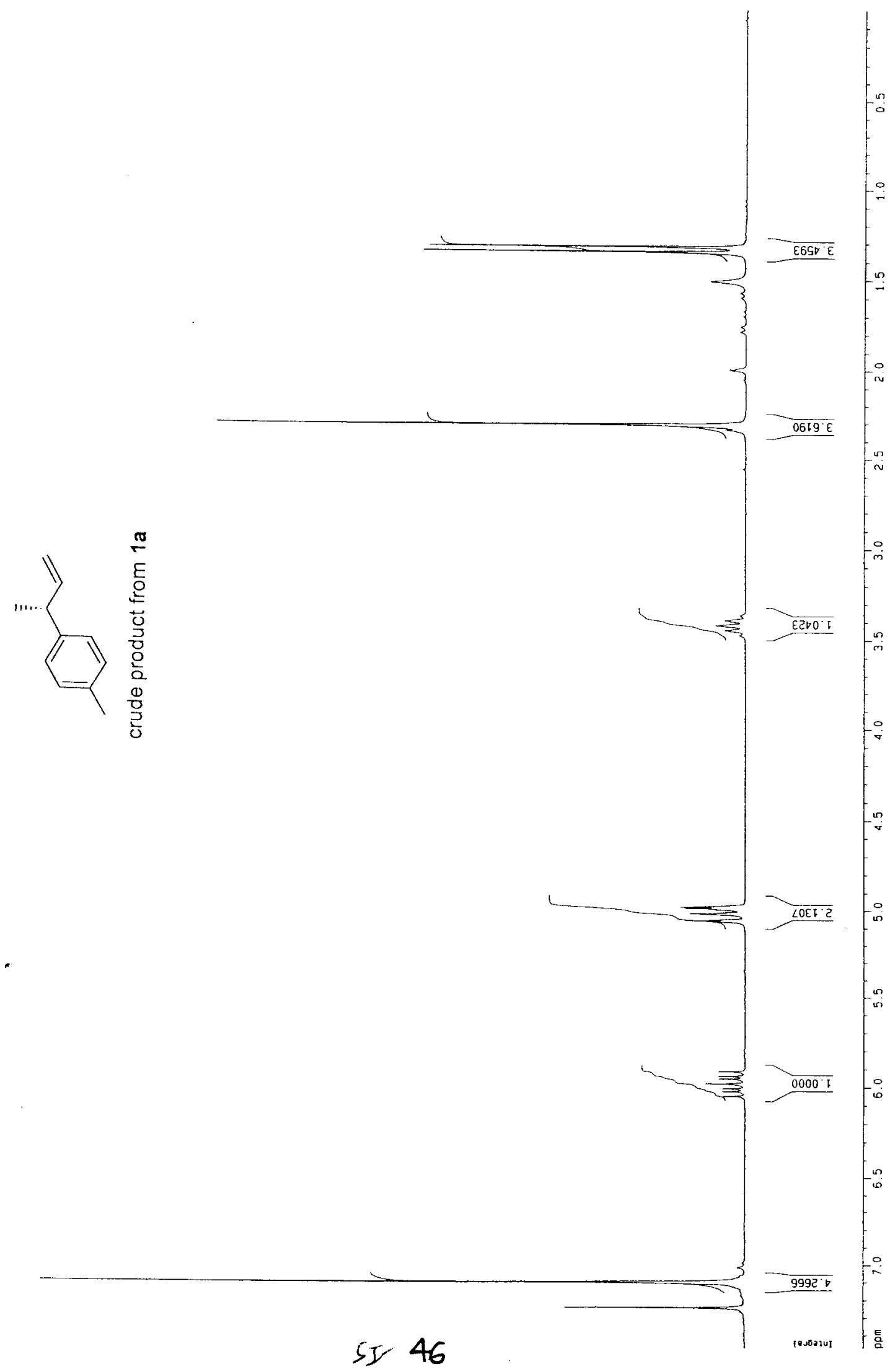



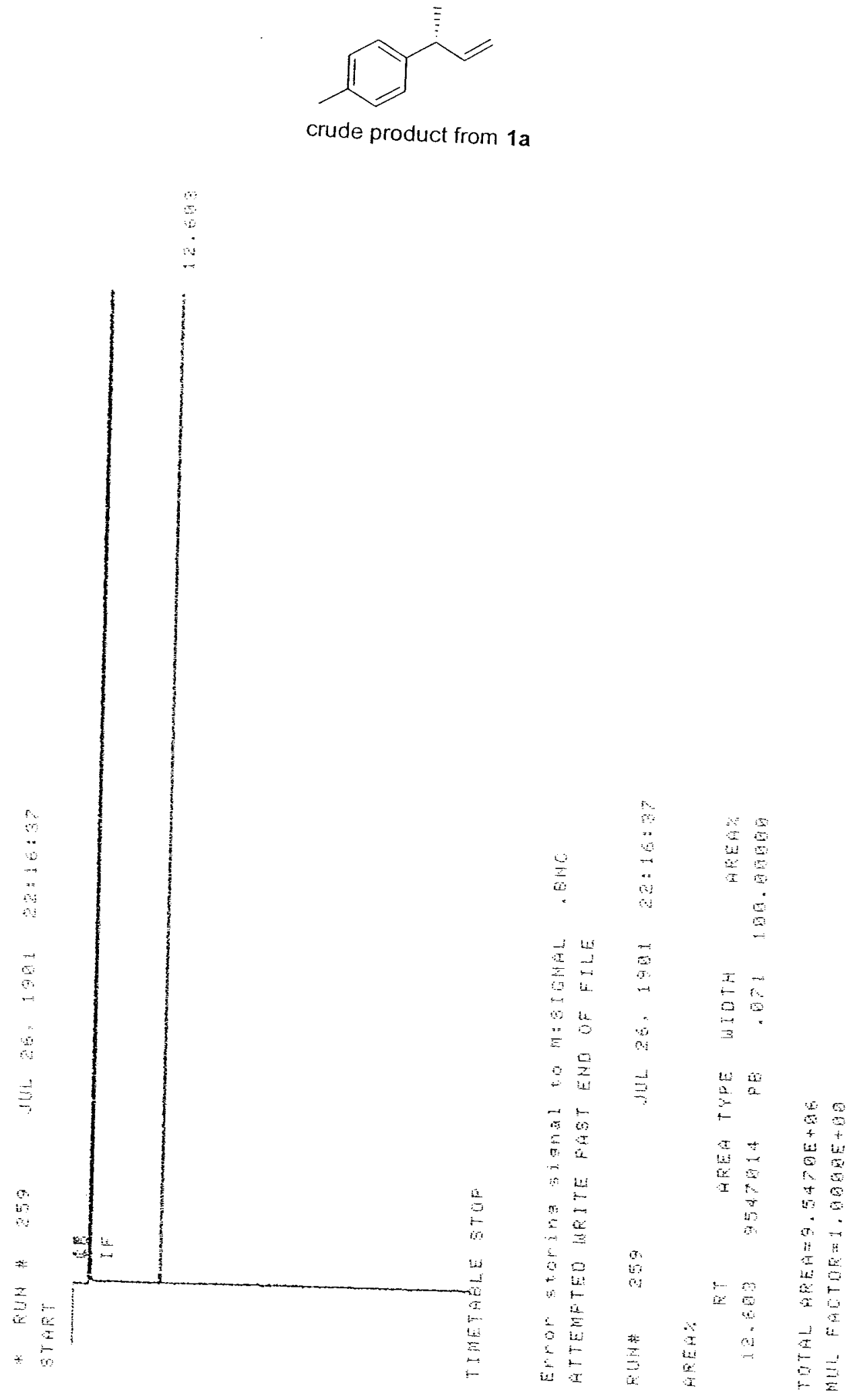

$1.0 \mathrm{~mL}$ helium $/ \mathrm{min}, 5 \mathrm{~min}$ at $80^{\circ} \mathrm{C}, 4^{\circ} \mathrm{C} / \mathrm{min}, 15 \mathrm{~min}$ at $180^{\circ} \mathrm{C}$ 

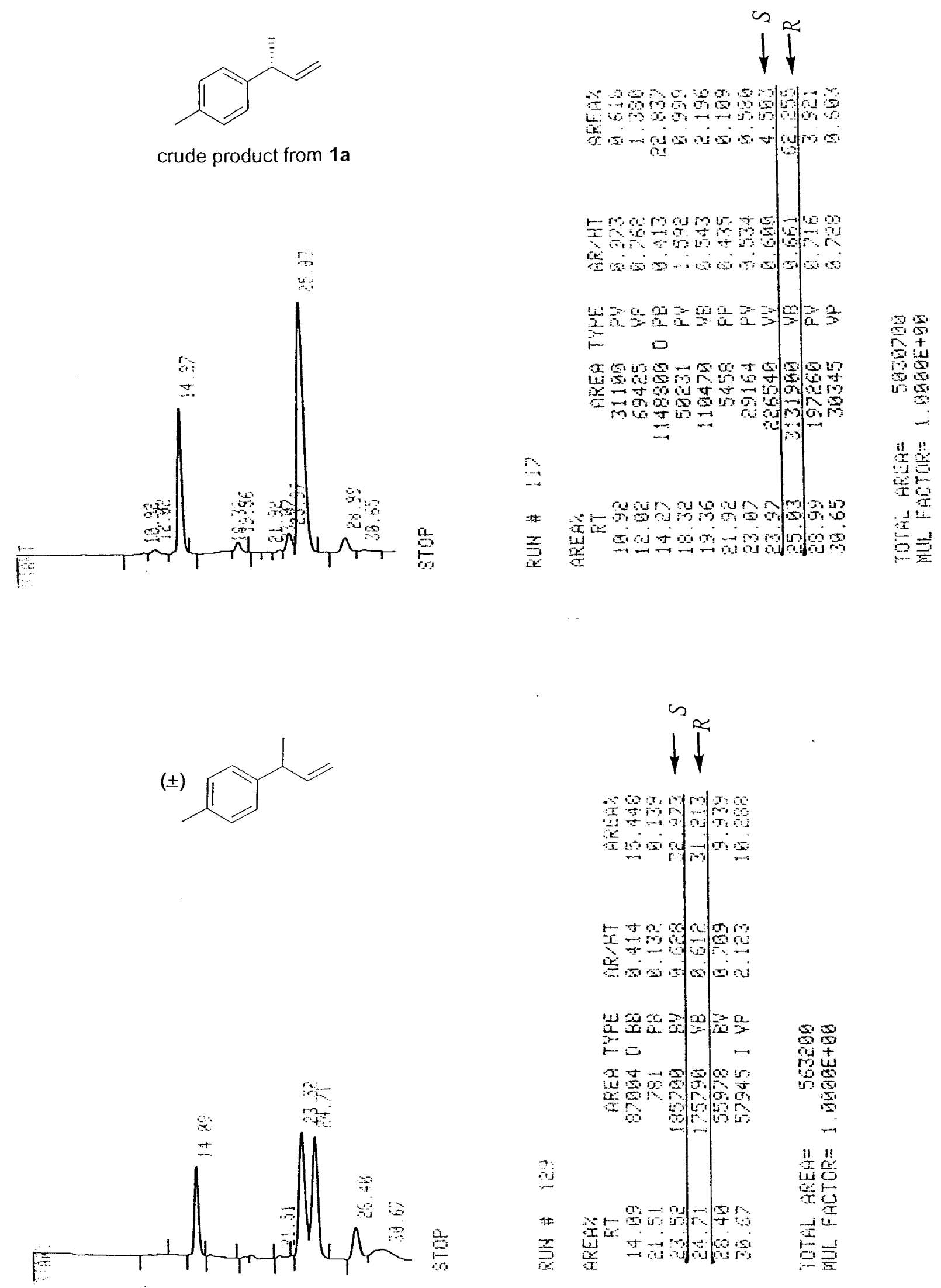

Daicel Chiralcel OJ Column, $100 \%$ hexane, $0.30 \mathrm{~mL} / \mathrm{min}$ 

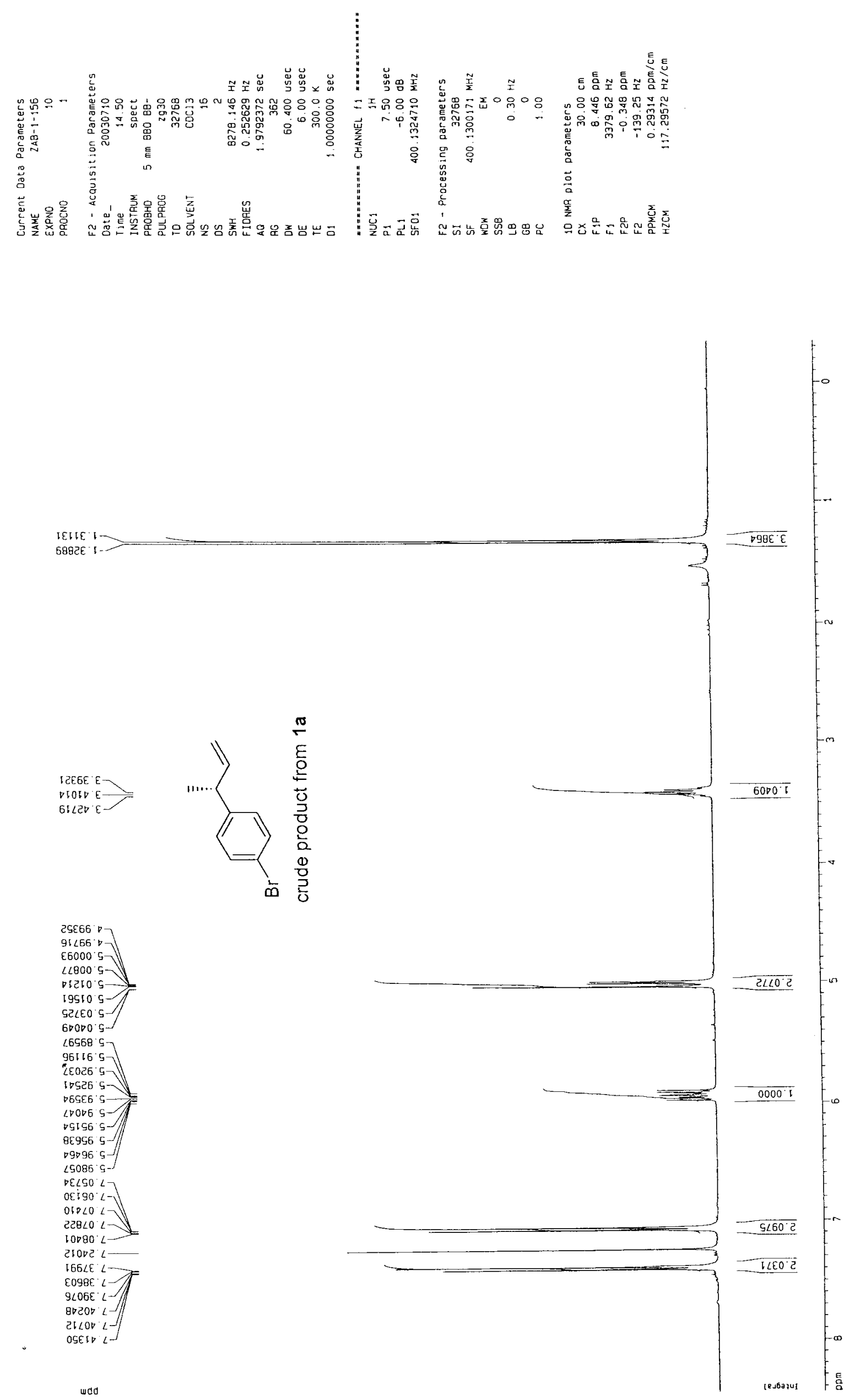

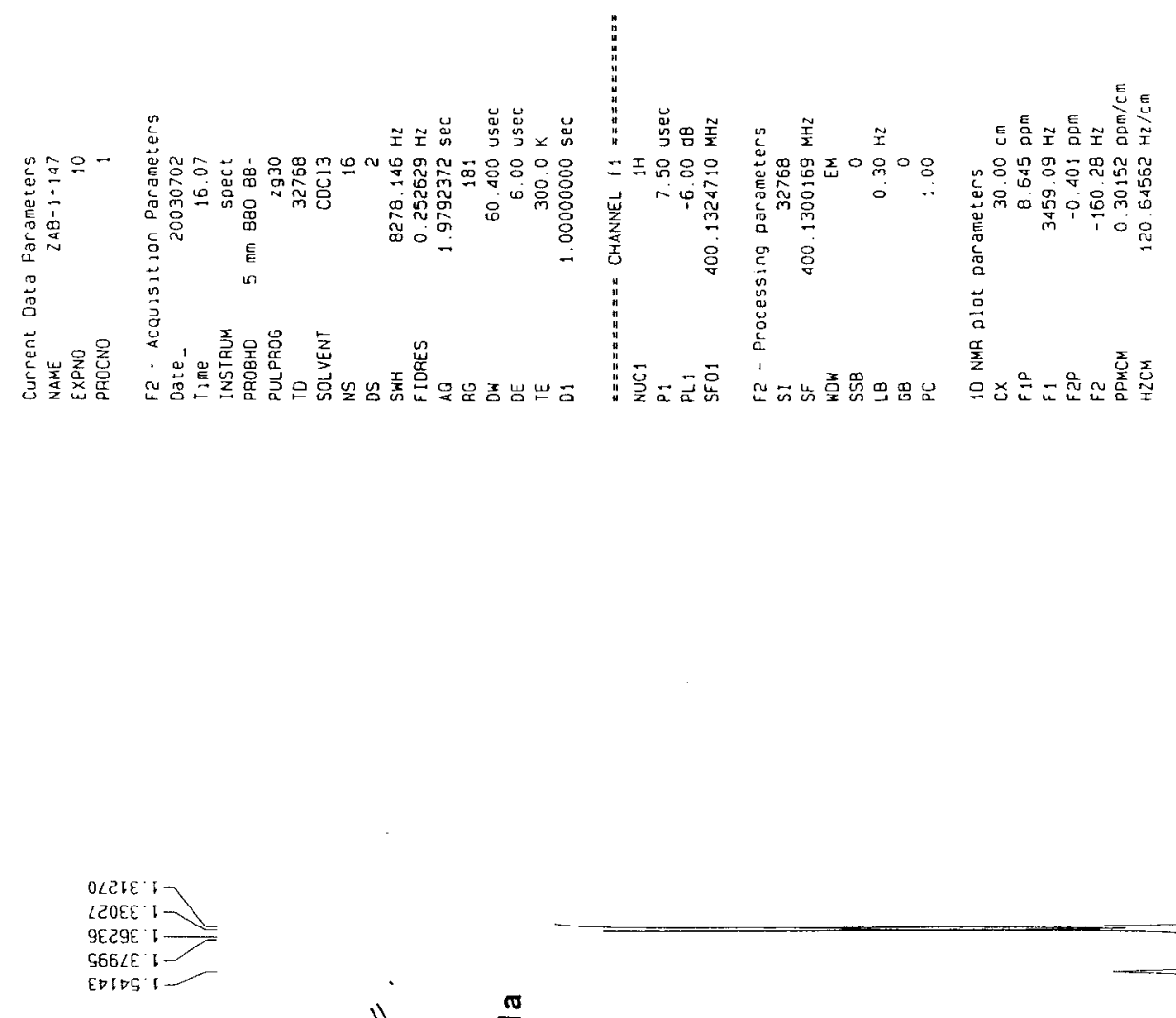

Er986 :-

$\angle 10052$

EOCLE E-

OE6BE $\varepsilon$
IE90大 $\varepsilon-1$

6टEटV $\varepsilon-1$

$686 \varepsilon \mathrm{V} \mathrm{E}$

OBZLLE-

$\angle 199 \angle \mathrm{C}-$

$\angle \angle S 6 L E-$

OECSOE

E59 65

โ5086 $\square$

$\left.8 \angle 586^{\circ} \cdot\right]$

$00200.5-$

LEG 0.5

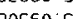

$92021.5=$

$0 \angle 695 \cdot 57$

6DEI9 9

ร0526 97

98056 $\mathrm{c}]$

CE 196 .

G6E86. $\mathrm{G}$

D9E66. 5

E $6600^{\circ} 9$

५ธI19. 9

हเ6Е9 9-

$68559 \cdot 97$

$60 E 89.9-1$

LEOCg 9-

IE9Eg ' 9

os $\cos 9-$

$\checkmark 1058.9$

$\angle 5998^{\circ} 9^{-}$

99E8B $9-$

SE $160^{\circ} \cdot 9-$

$\angle \angle E O S L^{-1}$

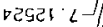

DE6Eᄅ $L J$

EL682 ¿

५टOटह $L$

[GIVE $L$

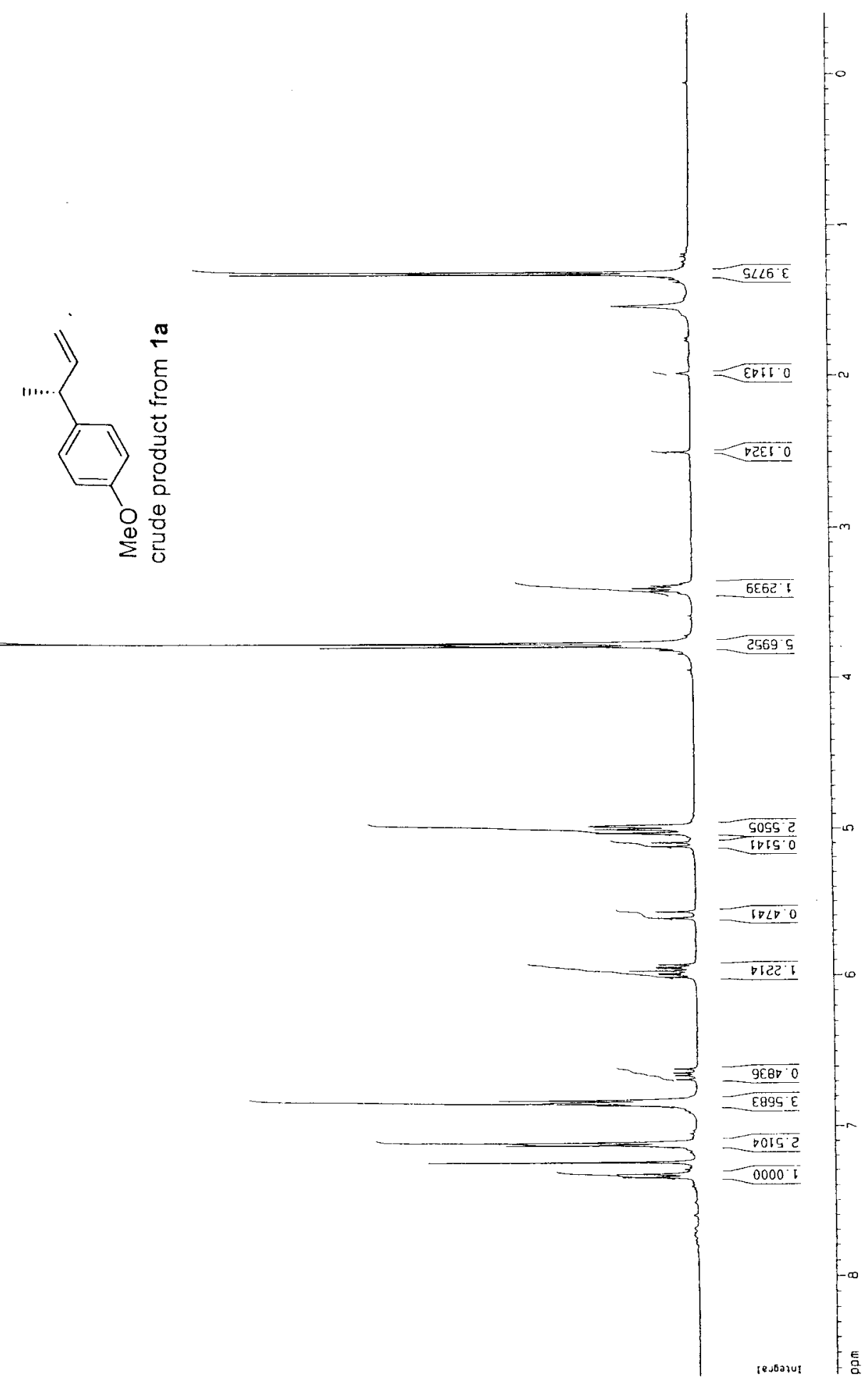

wdo 


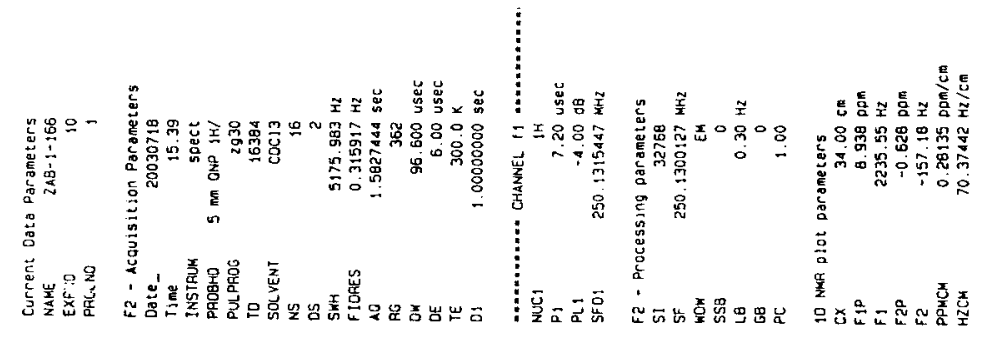

$\triangle \triangle G \angle O O$

9E952 5

00265.5

LLOE

EEZSE:

5502011

15800 1-

8EOrS $E$

$99995 \mathrm{E}-$

OSEGS E-

टा0ट० $\mathrm{E}-$

IE9०9. $\mathrm{E}-$

$\angle 9868^{\circ} \varepsilon-$

जE $\angle 06 E-$

96000's

$69080.5-7$

LSE5 . 9

LeLre 5

$\checkmark E \angle L C: S-$

20062: $\mathrm{s}$

BSE LL $S$

9BETS 5

ट८000 9

Bट0E0.9-

$68570.9-11$

टट2 $\angle 0.9-$

ᄂ $\angle 860.9$

OEDII. 9 -

9000 [ 9

85984.9

ट00EB $\cdot 9$

$8958 \cdot 9-$

BE006.9-

68101 2

EEIEI L

OBLEL $L-$

DQ6Eट ' $L$

$1266 \mathrm{C}^{\circ}<-7$

$\angle S E O E \cdot \angle-$

OQZEE $\angle-$

ICLEE $L-/ / F$

BICES $\angle-$

69E9S $\angle-1$

Lrobs $2-$

$\angle D 699^{\circ} L-$

$01899<-$

$\angle 1 E 6 \angle C$

I $8828<$

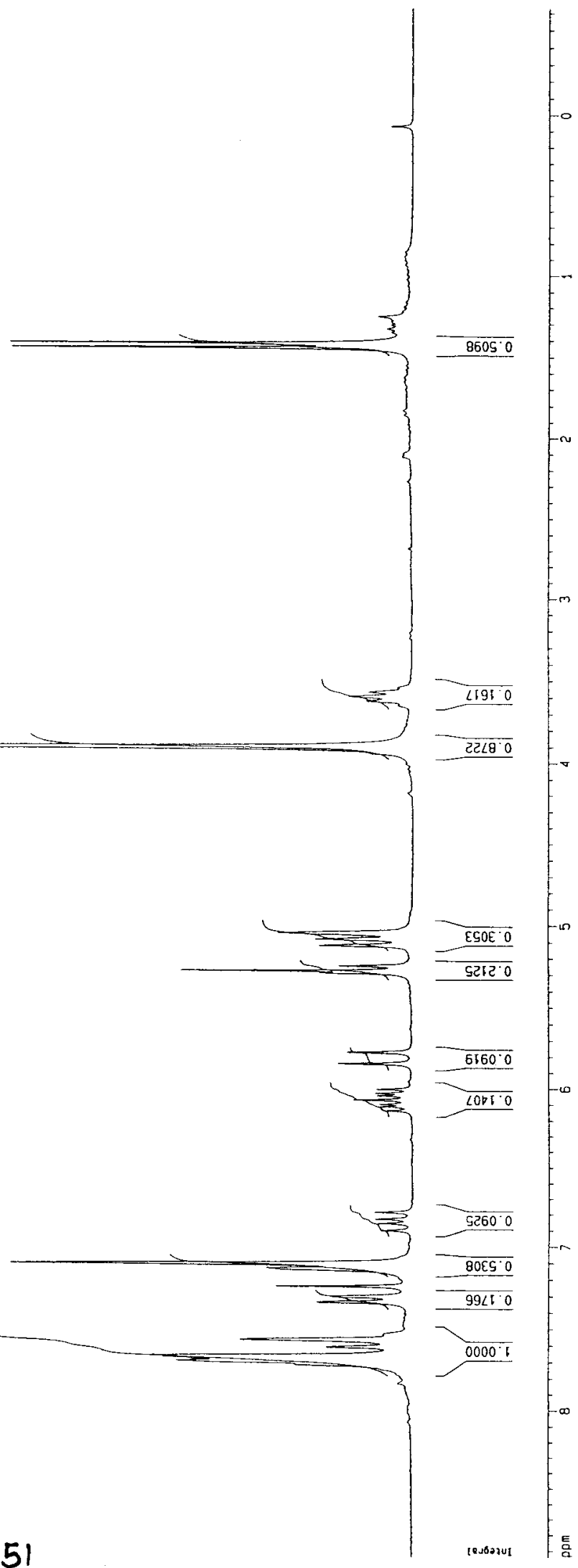




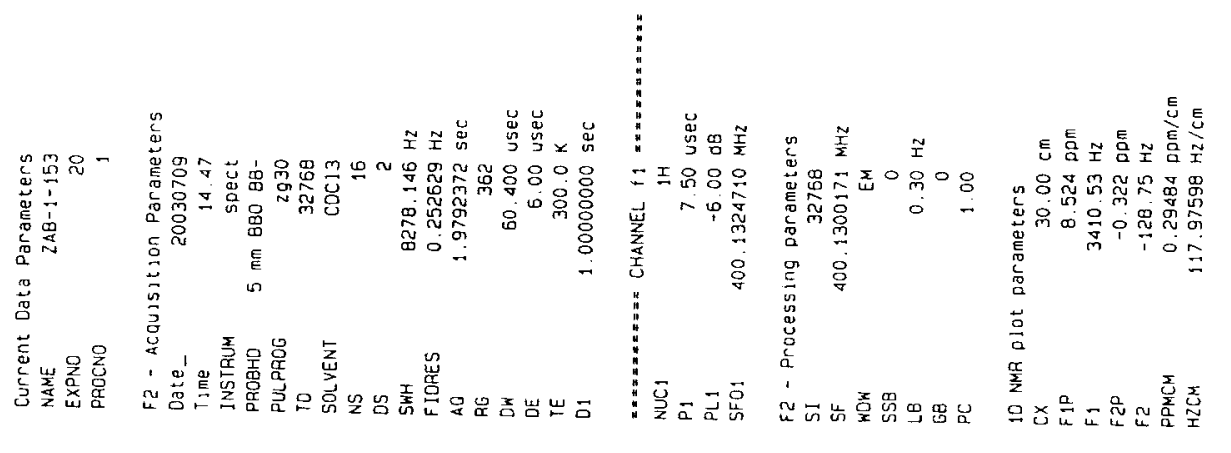

$\angle 9 \angle 500-\ldots$

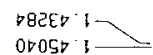

ODOSE

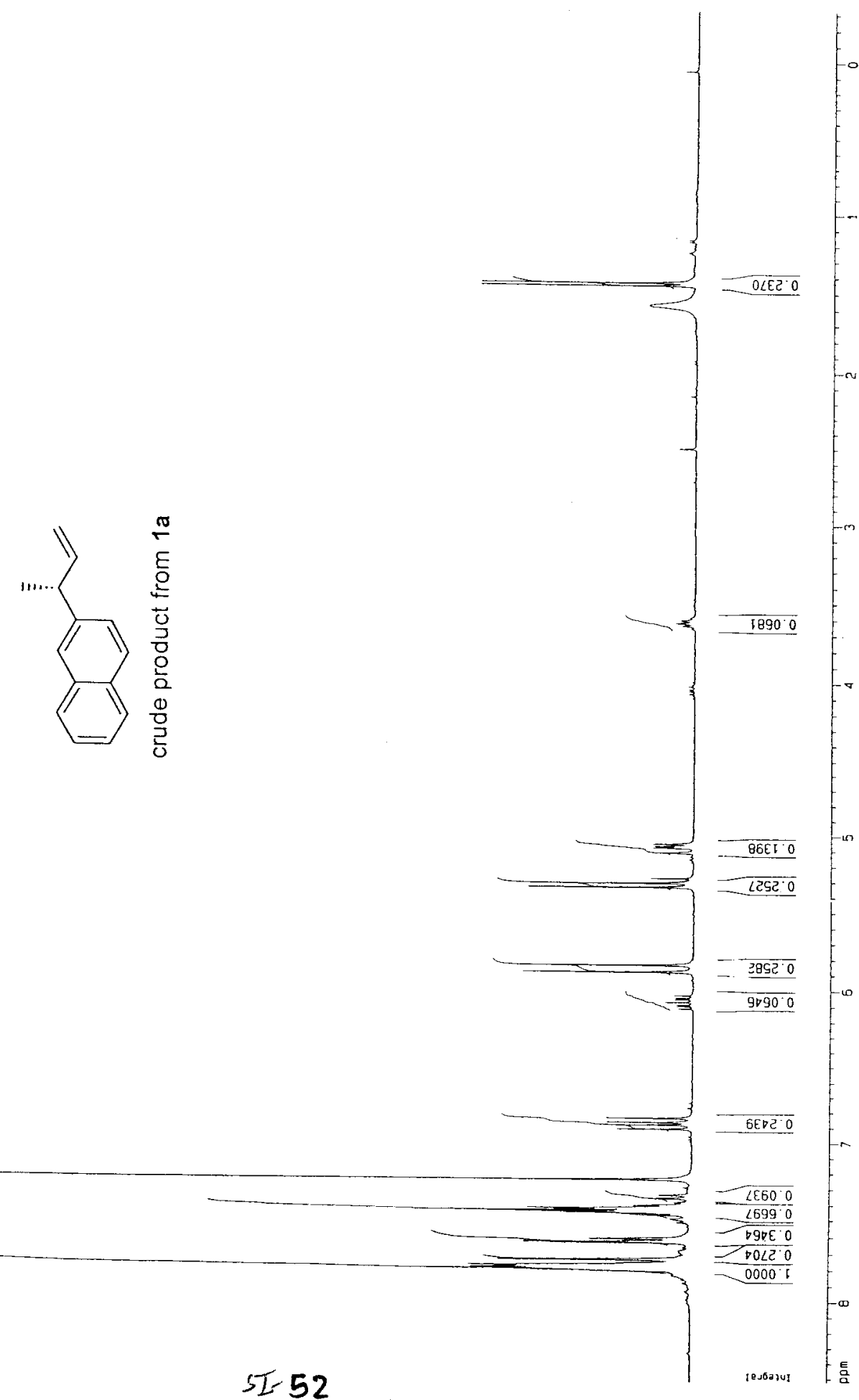

$91005 \cdot 2-$

$58509^{\circ} \mathrm{E}-$

65ट29 E-

$60009 \mathrm{E}$

$66290 \mathrm{~S}$

s9990 97

$15 \$ 90.5$

97890.57

$0<8<0^{\circ} \mathrm{G}$ )

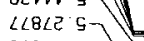

SISOE 9

LO60E $\mathrm{g}-\mathrm{C}$

GESEE $S$ -

Ьट9EE $5-$

259E8 $\mathrm{S}-$

C9LE 5

OE088 5

$1 \angle 188^{\circ} \mathrm{S}-$

E69E8. 9

To98 $9-$

$\angle 8088^{\circ} \cdot 9-$

80806 ' 97

E66E2 6$)$

BSEZ $\angle]$

$0 \angle \angle C D<J$

EZSE $\angle>$

S9ED $\angle>$

66900
$\angle S 150^{\circ} \angle$

I 6 II $\angle-$

SOIES $L$

6CSE9 $\angle-F$

gaSE $<<-/$

$010<6<$

$<$

टE $E 000: L]$

wdd 

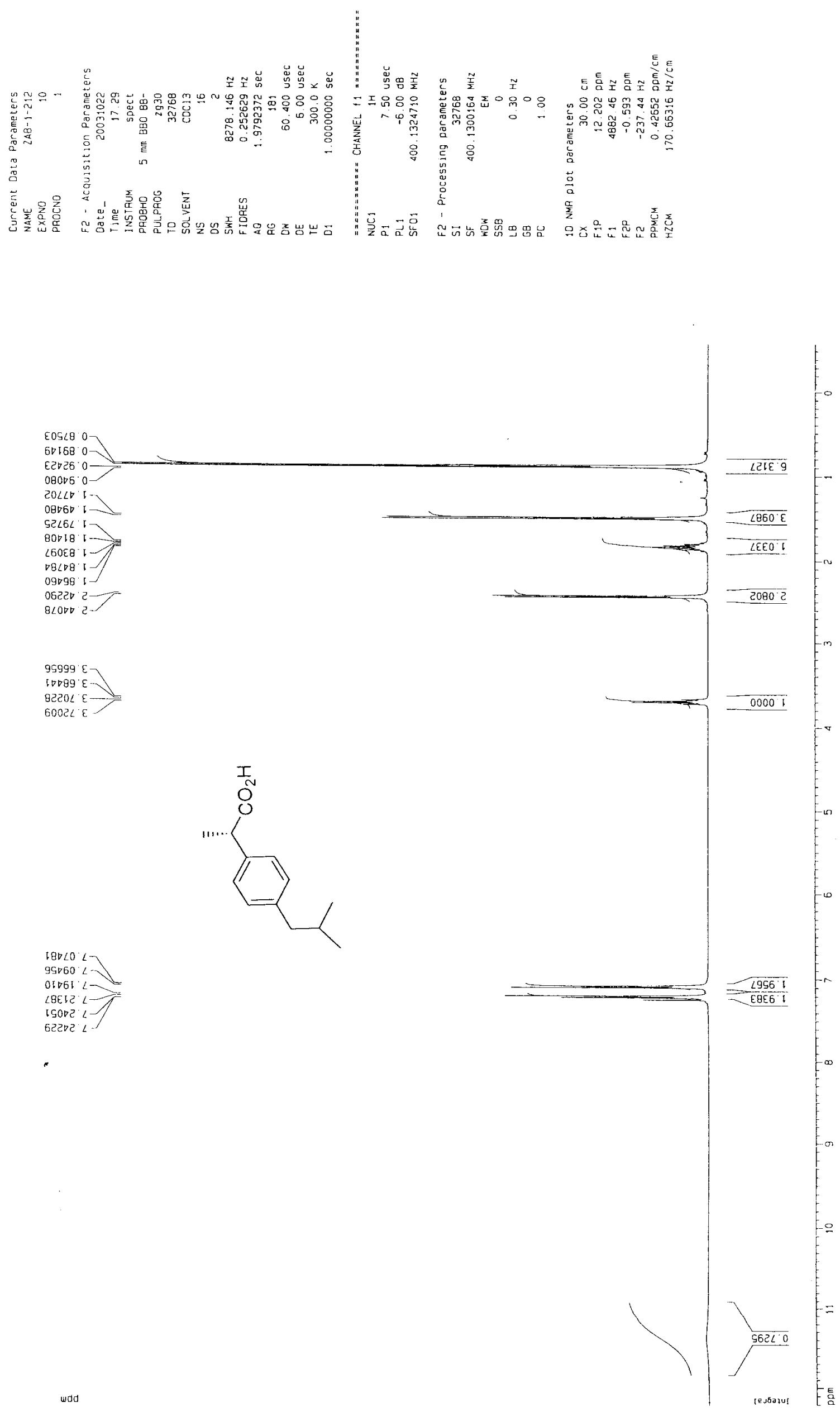

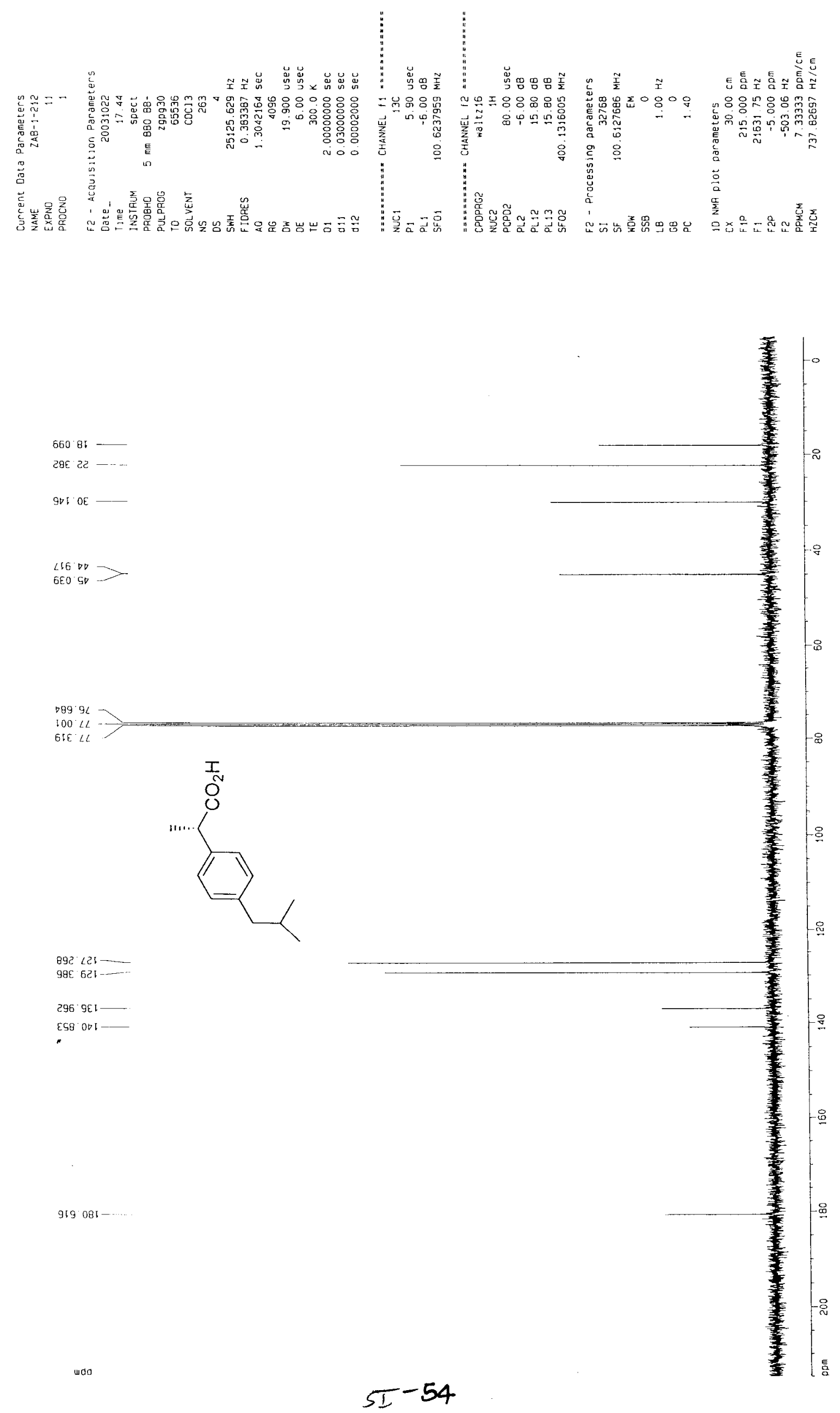

$895<51-\ldots$

296. $9 E$ L

ESQ O० 


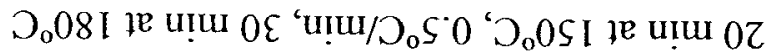

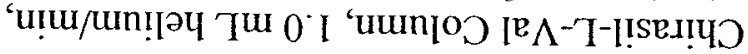

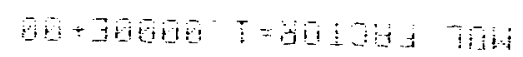

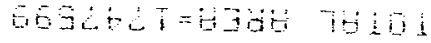

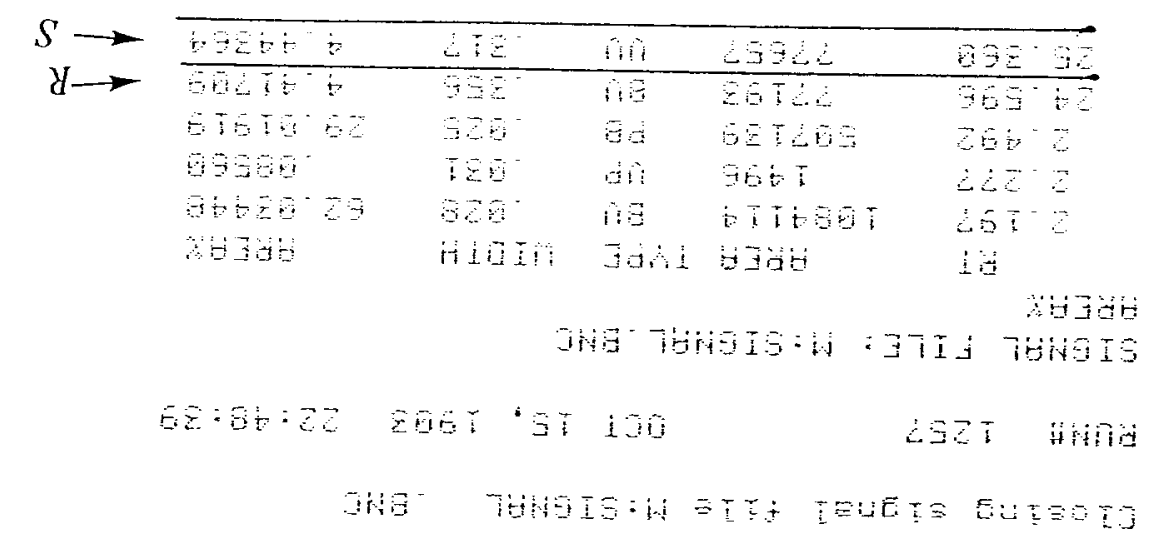
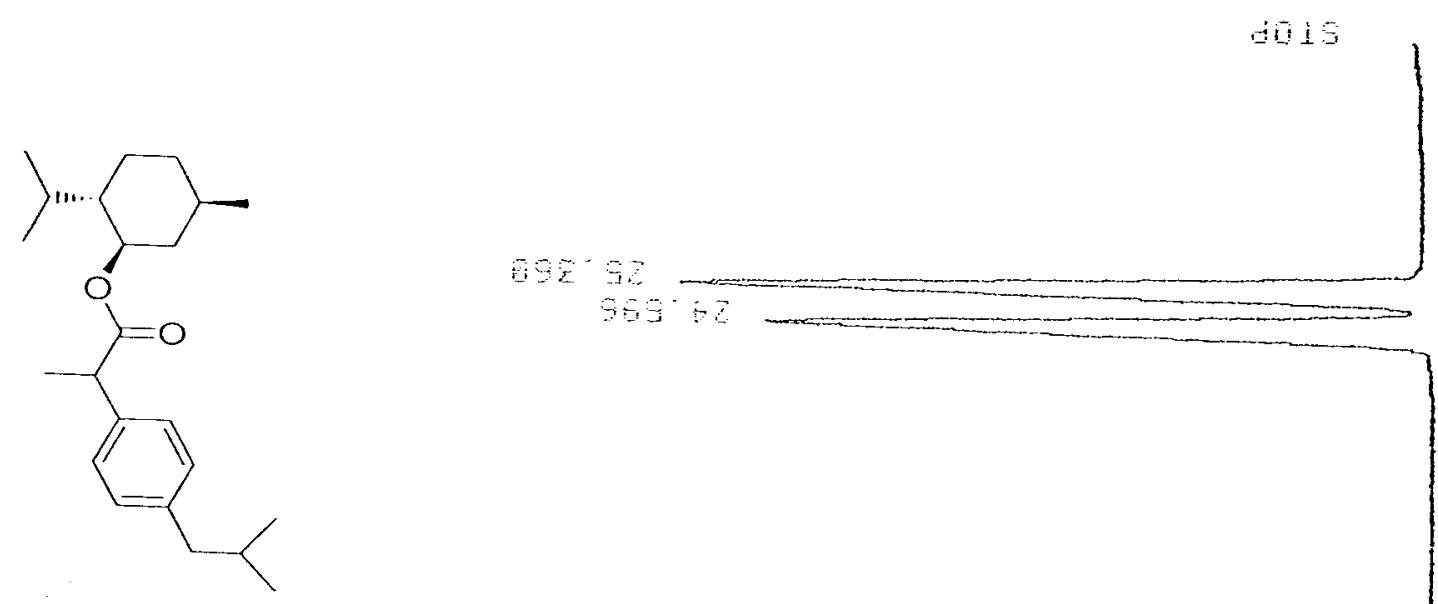

48

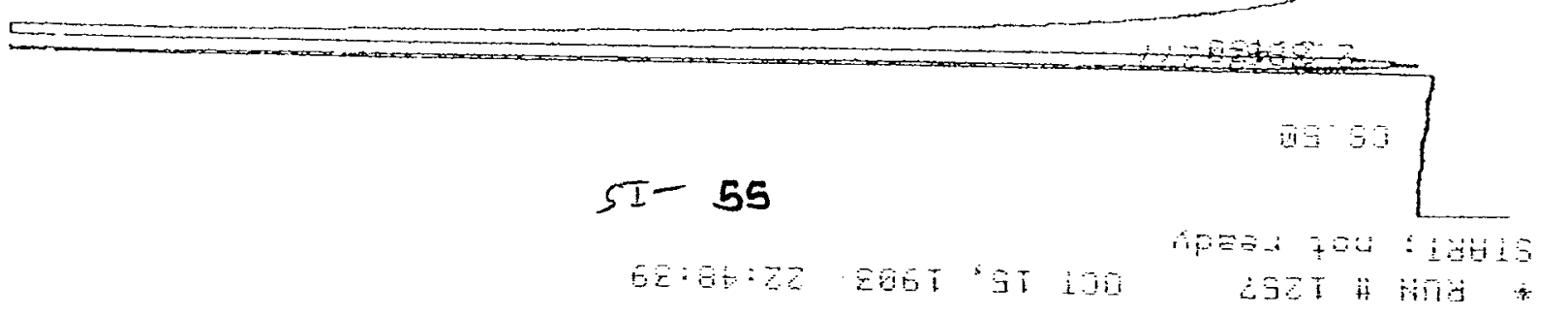




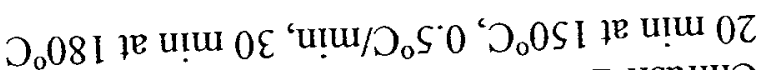

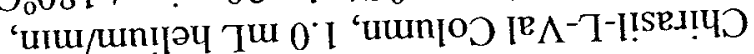

(5)

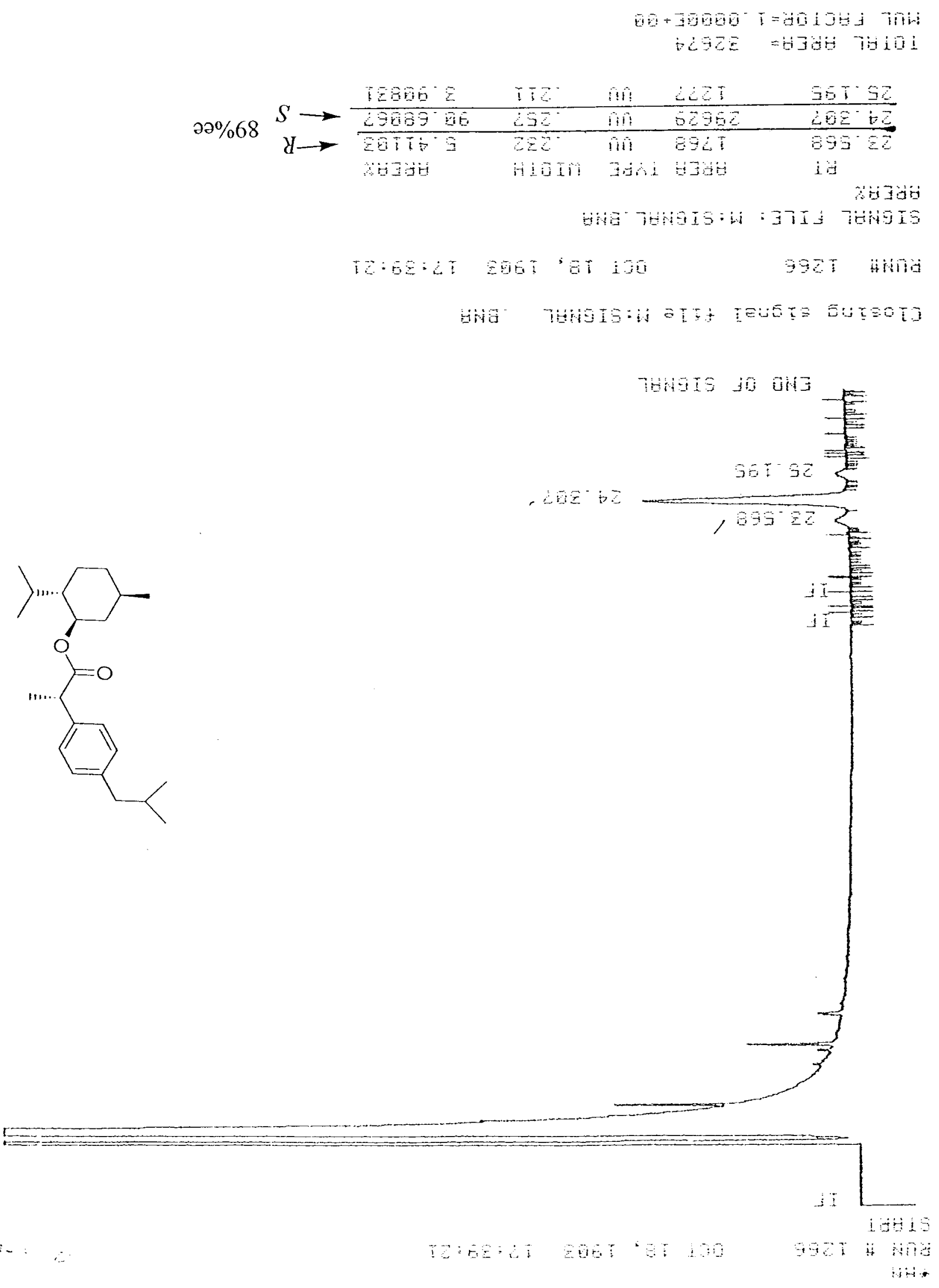



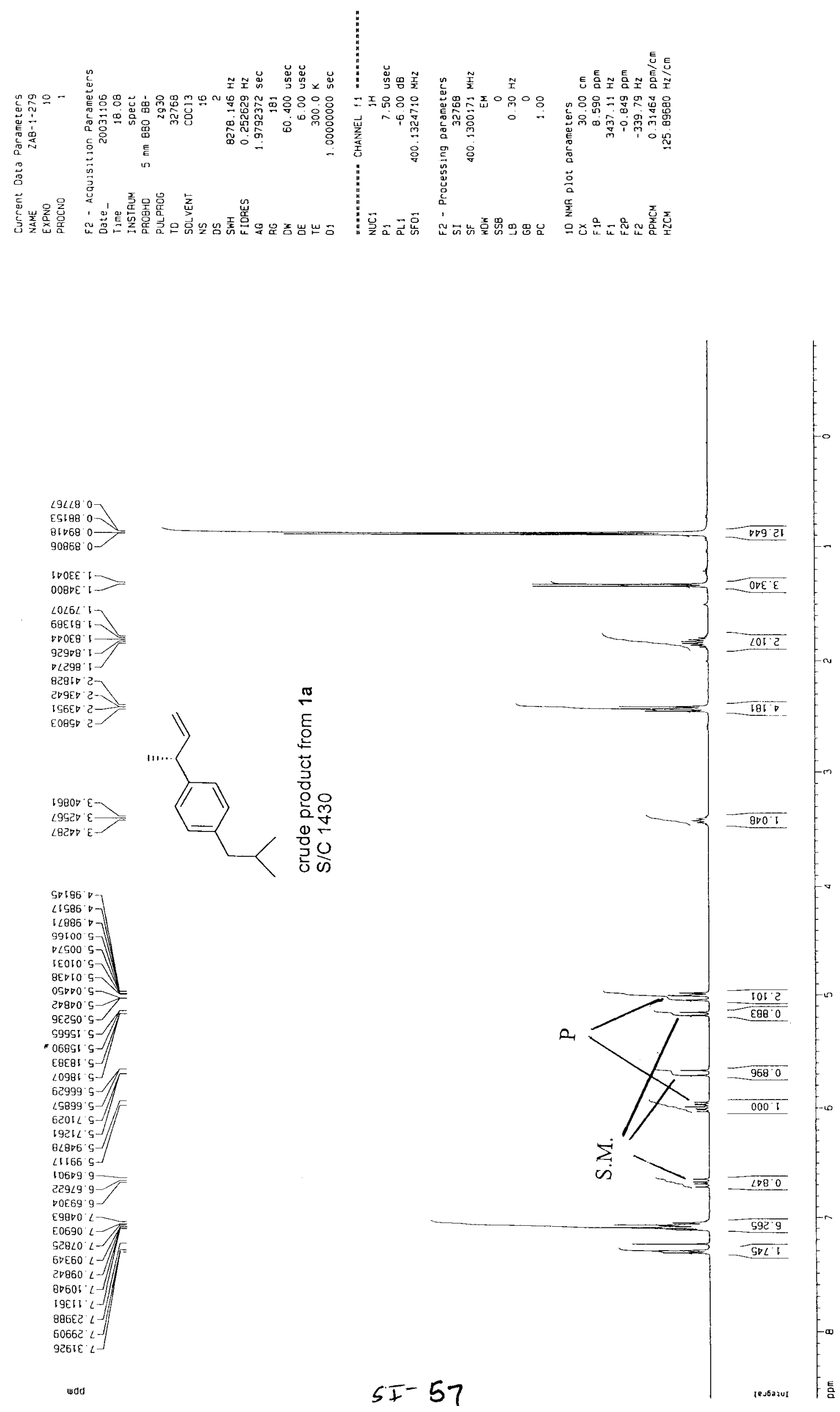


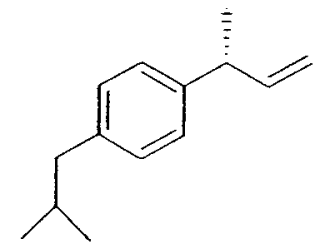

crude product from $1 \mathrm{a}$

S/C 1430

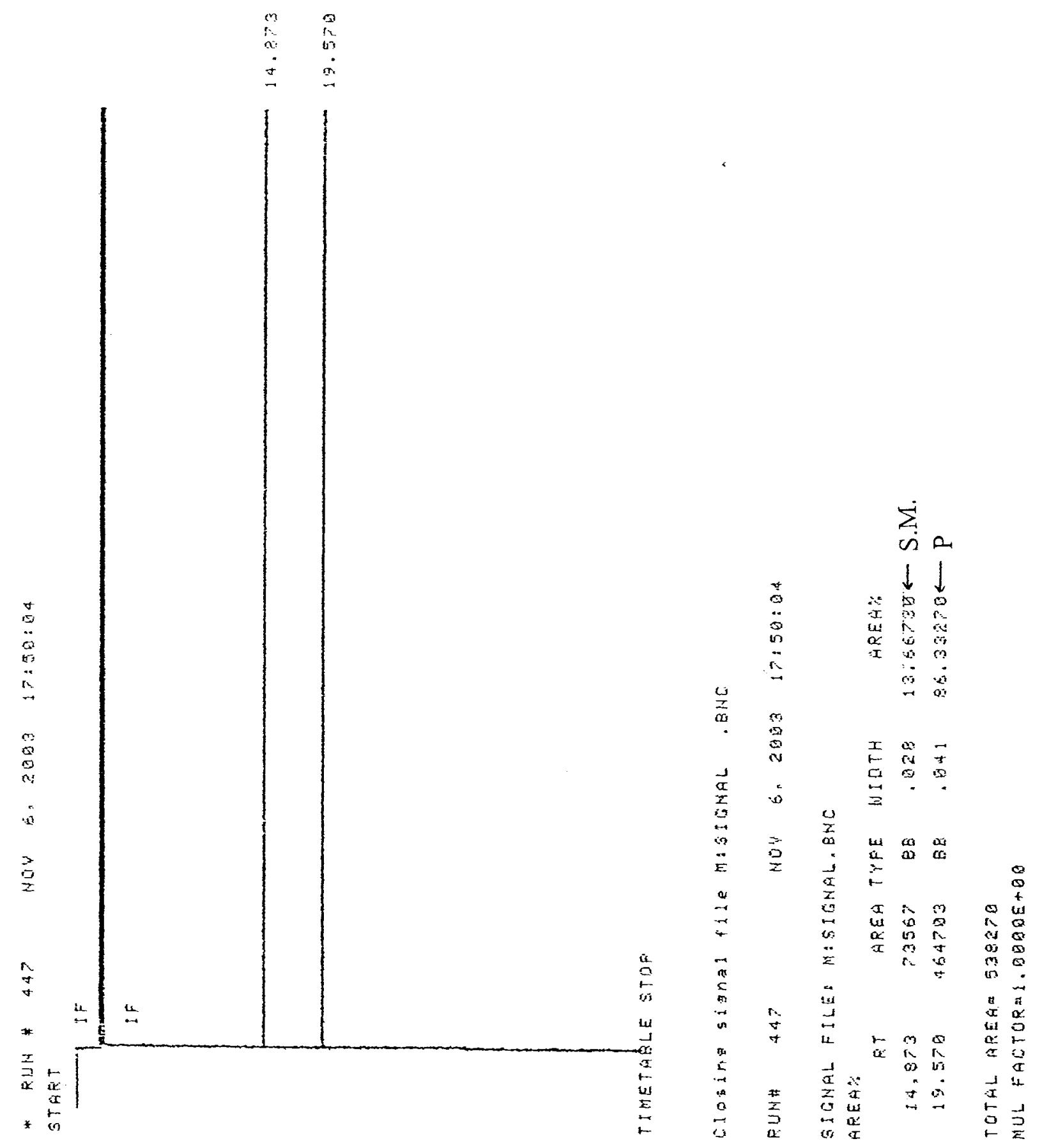

$1.0 \mathrm{~mL}$ helium $/ \mathrm{min}, 10 \mathrm{~min}$ at $100^{\circ} \mathrm{C}, 4^{\circ} \mathrm{C} / \mathrm{min}, 10 \mathrm{~min}$ at $200^{\circ} \mathrm{C}$ 


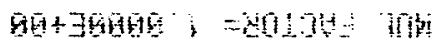

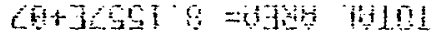

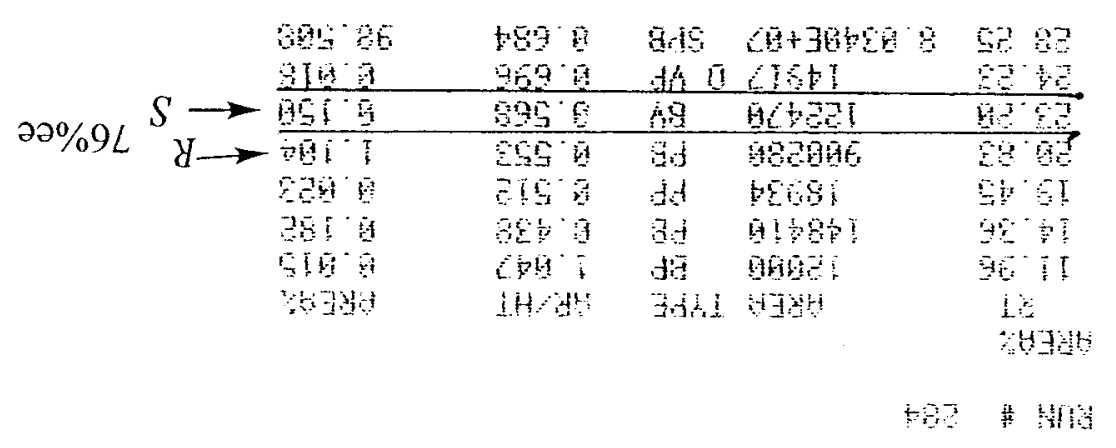

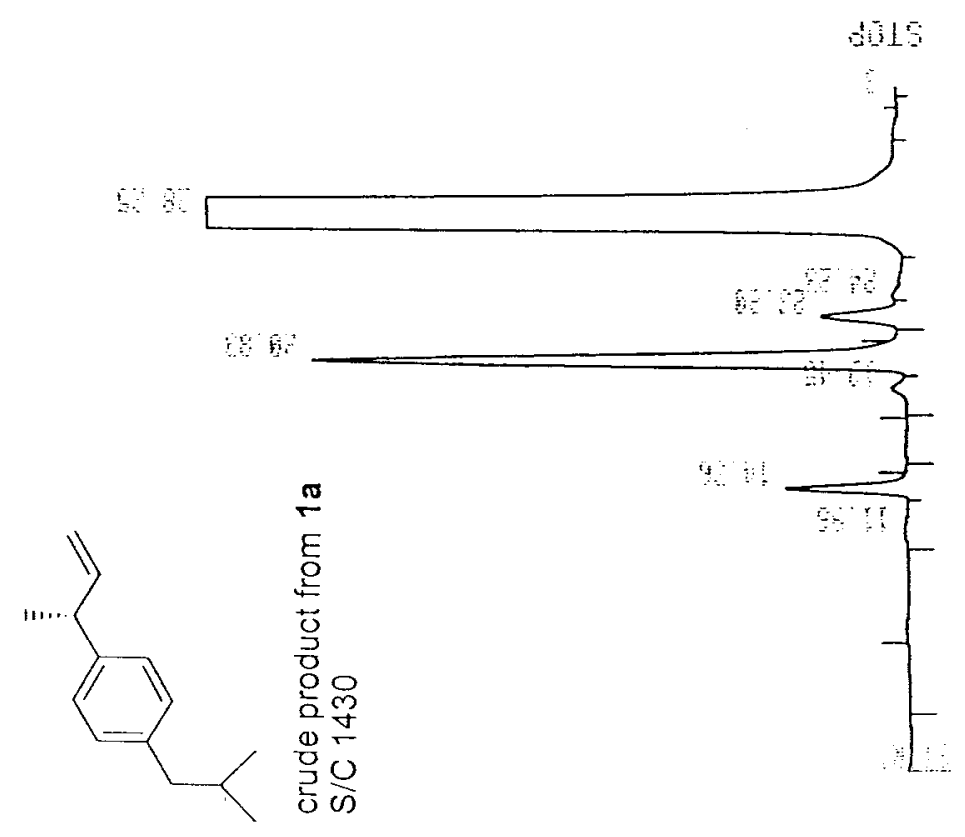




\section{Effect of Side-chain Chirality}

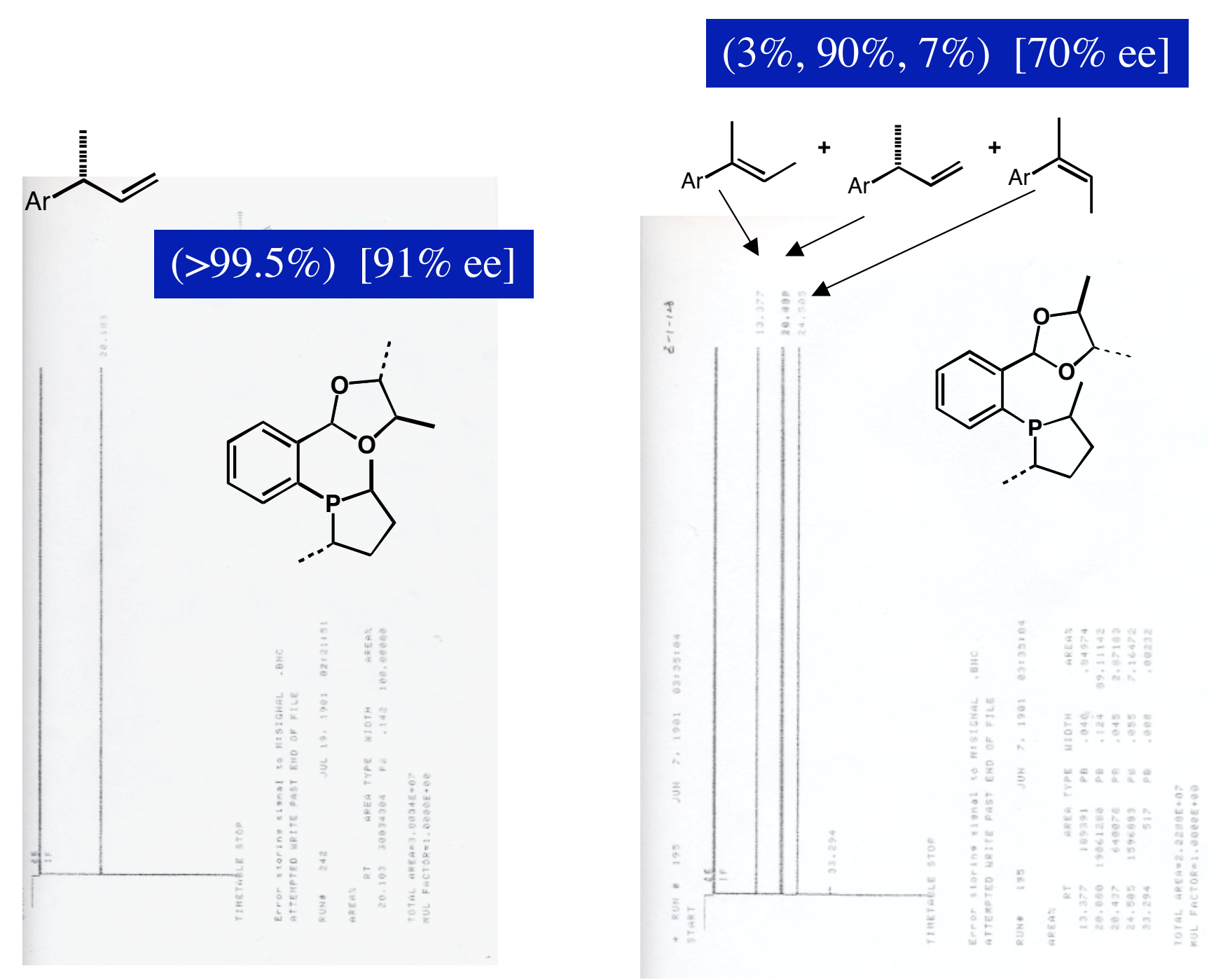

